\title{
Gravitational Waves from Gravitational Collapse
}

\author{
Chris L. Fryer \\ Los Alamos National Laboratory \\ MS B227, T-6 \\ Los Alamos, NM 87545 \\ U.S.A. \\ email: fryer@lanl.gov \\ Kimberly C. B. New \\ Los Alamos National Laboratory \\ MS T085, X-2 \\ Los Alamos, NM 87545 \\ U.S.A. \\ email: knew@lanl.gov \\ Accepted on 13 January 2003 \\ Published on 10 March 2003 \\ Living Reviews in Relativity \\ Published by the \\ Max Planck Institute for Gravitational Physics \\ (Albert Einstein Institute) \\ Am Mühlenberg 1, 14424 Golm, Germany \\ ISSN 1433-8351
}

\begin{abstract}
Gravitational wave emission from stellar collapse has been studied for more than three decades. Current state-of-the-art numerical investigations of collapse include those that use progenitors with more realistic angular momentum profiles, properly treat microphysics issues, account for general relativity, and examine non-axisymmetric effects in three dimensions. Such simulations predict that gravitational waves from various phenomena associated with gravitational collapse could be detectable with ground-based and space-based interferometric observatories. This review covers the entire range of stellar collapse sources of gravitational waves: from the accretion induced collapse of a white dwarf through the collapse down to neutron stars or black holes of massive stars to the collapse of supermassive stars.
\end{abstract}

This 2006 revision was written by Chris L. Fryer and Kim New jointly.

(c) Max Planck Society and the authors.

Further information on copyright is given at http://relativity.livingreviews .org/About/copyright.html For permission to reproduce the article please contact livrev@aei.mpg.de. 


\section{How to cite this article}

Owing to the fact that a Living Reviews article can evolve over time, we recommend to cite the article as follows:

Chris L. Fryer and Kimberly C. B. New,

"Gravitational Waves from Gravitational Collapse",

Living Rev. Relativity, 6, (2003), 2. [Online Article]: cited [<date $>$ ],

http://www.livingreviews.org/lrr-2003-2

The date given as $<$ date $>$ then uniquely identifies the version of the article you are referring to. 


\section{Article Revisions}

Living Reviews supports two different ways to keep its articles up-to-date:

Fast-track revision A fast-track revision provides the author with the opportunity to add short notices of current research results, trends and developments, or important publications to the article. A fast-track revision is refereed by the responsible subject editor. If an article has undergone a fast-track revision, a summary of changes will be listed here.

Major update A major update will include substantial changes and additions and is subject to full external refereeing. It is published with a new publication number.

For detailed documentation of an article's evolution, please refer always to the history document of the article's online version at http://www.livingreviews.org/lrr-2003-2.

24 Jan 2006: This submission is a revision to the "Gravitational Waves from Gravitational Collapse" review by Kim New - it was written by Chris L. Fryer and Kim New jointly. This revision focused mostly on updating the past review and adding more movies. The major changes are as follows:

Page 7: Revised paragraph above and added references.

Page 9: Revised section.

Page 9: Revised section, added Figure 1 and reference.

Page 10: Revised section and added references.

Page 11: Added reference.

Page 16: Revised section and added references.

Page 18: Revised section.

Page 19: Nakamura's 2D simulations moved to Section 4.3.

Page 22: Added reference.

Page 22: Added references.

Page 23: Added references.

Page 25: Extended paragraph above.

Page 25: Updated references.

Page 26: Added movie by Ou et al. (2004)

Page 27: Removed paragraph on Davies et al. (2002).

Page 27: Added reference.

Page 30: Shibata's simulations moved to Section 4.3.

Page 30: Revised section and added Figures 12 and 13.

Page 32: Added references.

Page 35: Added movie.

Page 36: Revised paragraphs above and added Movie 17.

Page 36: Added reference. 
Page 37: We have taken pieces of the "Collapse of Massive Stars" section to develop a "Collapsar" section.

Page 40: Revised paragraph below and added reference.

Page 44: Added reference.

Page 45: Updated summary. 


\section{Contents}

1 Introduction $r$

2 Accretion Induced Collapse $\quad 9$

2.1 Collapse scenario . . . . . . . . . . . . . . . . . . . . . . . . . . . . . . . . . . . . . . .

2.2 Formation rate . . . . . . . . . . . . . . . . . . . . . 9

2.3 GW emission mechanisms . . . . . . . . . . . . . . . . . . . . 10

2.4 Numerical predictions of GW emission . . . . . . . . . . . . . . . . . . . 11

2.4.1 Full collapse simulations . . . . . . . . . . . . . . . . . . 11

2.4.2 Analyses of equilibrium representations of collapse . . . . . . . . . . 14

2.5 Going further . . . . . . . . . . . . . . . . . . . 15

3 Collapse of Massive Stars 16

3.1 Collapse scenario . . . . . . . . . . . . . . . . . . . . . 16

3.2 Formation rate . . . . . . . . . . . . . . . . . . . . 18

3.3 GW emission mechanisms . . . . . . . . . . . . . . . . . . . . . 18

3.4 Numerical predictions of GW emission . . . . . . . . . . . . . . . . . . . . . 18

3.4 Historical investigations . . . . . . . . . . . . . . . . 18

3.4 .2 Axisymmetric simulations . . . . . . . . . . . . . . . . 19

3.4.3 Non-axisymmetric simulations . . . . . . . . . . . . . . . . . . . . . . . . . . . . . .

3.4.4 General relativistic simulations . . . . . . . . . . . . . . . . . 25

3.4.5 Simulations of convective instabilities . . . . . . . . . . . . . 30

3.5 Going further . . . . . . . . . . . . . . . . . . 36

4 Collapsar Models $\quad \mathbf{3 7}$

4.1 Collapse scenario . . . . . . . . . . . . . . . . . . . . . . . . . . . . . . . . . . . .

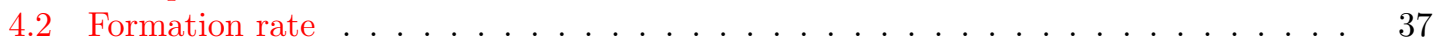

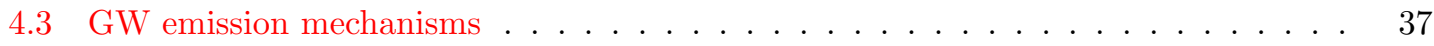

4.4 Numerical Predictions of GW emission Mechanisms . . . . . . . . . . . . . . . . 38

4.5 Going further . . . . . . . . . . . . . . . . . . . 39

5 Collapse of Population III Stars $\quad 40$

5.1 Collapse scenario . . . . . . . . . . . . . . . . . . . . . . . . . . . . . 40

5.2 Formation rate . . . . . . . . . . . . . . . . . . . . . 40

5.3 GW emission mechanisms . . . . . . . . . . . . . . . . . . . . . . 40

5.4 Numerical predictions of GW emission . . . . . . . . . . . . . . . . . 40

6 Collapse of Supermassive Stars $\quad 42$

6.1 Collapse scenario . . . . . . . . . . . . . . . . . . . . . . . 42

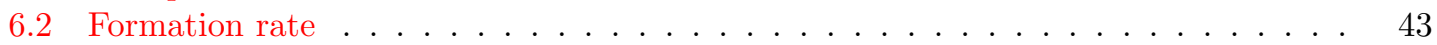

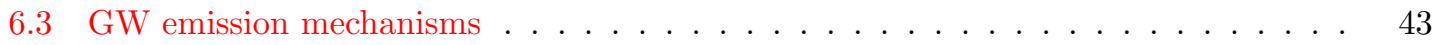

6.4 Numerical predictions of GW emission . . . . . . . . . . . . . . . . 43

7 Summary $\quad 45$

8 Acknowledgements $\quad 48$

$\begin{array}{lr}\text { References } & 68\end{array}$ 



\section{Introduction}

The field of gravitational wave $(\mathrm{GW})$ astronomy will soon become a reality. The first generation of ground-based interferometric detectors (LIGO [45], VIRGO [126], GEO 600 [104], TAMA 300 [182]) are beginning their search for GWs. Toward the end of this decade, two of these detectors (LIGO, VIRGO) will begin upgrades that should allow them to reach the sensitivities necessary to regularly detect emissions from astrophysical sources. A space-based interferometric detector, LISA [181], could be launched in the early part of the next decade. One important class of sources for these observatories is stellar gravitational collapse. This class covers an entire spectrum of stellar masses, from the accretion induced collapse (AIC) of a white dwarf through the collapse of massive stars $\left(M>8 M_{\odot}\right)$ including the "collapsar" engine believed to power long-duration gamma-ray bursts [261], very massive Population III stars $\left(M=100-500 M_{\odot}\right)$, and supermassive stars (SMSs, $M>10^{6} M_{\odot}$ ). Some of these collapses result in explosions (Type II, Ib/c supernovae and hypernovae) and all leave behind neutron star or black hole remnants.

Strong GWs can be emitted during a gravitational collapse/explosion and, following the collapse, by the resulting compact remnant [244, 172, 173, 73, 214, 91, 88, 120]. GW emission during the collapse itself may result if the collapse or explosion involves aspherical bulk mass motion or convection. Rotational or fragmentation instabilities encountered by the collapsing star will also produce GWs. Asymmetric neutrino emission can also produce a strong gravitational wave signature. Neutron star remnants of collapse may emit GWs due to the growth of rotational or $r$-mode instabilities. Black hole remnants will also be sources of GWs if they experience accretion induced ringing or if the disks around the black hole develop instabilities. All of these phenomena have the potential of being detected by gravitational wave observatories because they involve the rapid change of dense matter distributions.

Observation of gravitational collapse by gravitational wave detectors will provide unique information, complementary to that derived from electromagnetic and neutrino detectors. Gravitational radiation arises from the coherent superposition of mass motion, whereas electromagnetic emission is produced by the incoherent superposition of radiation from electrons, atoms, and molecules. Thus, GWs carry different kinds of information than other types of radiation. Furthermore, electromagnetic radiation interacts strongly with matter and thus gives a view of the collapse only from lower density regions near the surface of the star, and it is weakened by absorption as it travels to the detector. In contrast, gravitational waves can propagate from the innermost parts of the stellar core to detectors without attenuation by intervening matter. With their weak interaction cross-sections, neutrinos can probe the same region probed by GWs. But whereas neutrinos are extremely sensitive to details in the microphysics (equation of state and cross-sections), GWs are most sensitive to physics driving the mass motions (e.g., rotation). Combined, the neutrino and the GW signals can teach us much about the conditions in the collapsing core and ultimately the physics that governs stellar collapse (e.g., [7, 87]).

The characteristics of the GW emission from gravitational collapse have been the subject of much study. Core collapse supernovae, in particular, have been investigated as sources of gravitational radiation for more than three decades (see, e.g., [203, 245, 204, 57, 179, 171, 233, 74, 170, $271,198,86,88]$ ). However, during this time research has produced estimates of GW strength that vary over orders of magnitude. This is due to the complex nature of core collapse. Important theoretical and numerical issues include

- construction of accurate progenitor models, including realistic angular momentum distributions,

- proper treatment of microphysics, including the use of realistic equations of state and neutrino transport,

- simulation in three-dimensions to study non-axisymmetric effects, 
- inclusion of general relativistic effects,

- inclusion of magnetic field effects, and

- study of the effect of an envelope on core behavior.

To date, collapse simulations generally include state-of-the-art treatments of only one or two of the above physics issues (often because of numerical constraints). For example, those studies that include advanced microphysics have often been run with Newtonian gravity (and approximate evaluation of the GW emission; see Section 2.4). A 3D, general relativistic collapse simulation that includes all significant physics effects is not feasible at present. However, good progress has been made on the majority of the issues listed above; the more recent work will be reviewed in some detail here.

The remainder of this article is structured as follows. Each category of gravitational collapse will be discussed in a separate section (AIC in Section 2, collapse of massive stars in Section 3, collapsar models in Section 4, collapse of Population III stars in Section 5, and collapse of SMSs in Section 6). Each of these sections $(2,3,4,5,6)$ is divided into subsection topics: collapse scenario, formation rate, GW emission mechanisms, and numerical predictions of GW emission. In the subsections on numerical predictions, the detectability of the GW emission from various phenomena associated with collapse is examined. In particular, the predicted characteristics of GW emission are compared to the sensitivities of LIGO (for sources with frequencies of 1 to $10^{4} \mathrm{~Hz}$ ) and LISA (for sources with lower frequencies in the range of $10^{-4}$ to $1 \mathrm{~Hz}$ ).

Living Reviews in Relativity

http://www. livingreviews. org/lrr-2003-2 


\section{Accretion Induced Collapse}

\subsection{Collapse scenario}

Stars with masses below $8 M_{\odot}$ end their lives ejecting their envelopes in a possible planetary nebula, leaving behind a white dwarf that gradually cools and fades away. For those white dwarfs in binaries, binary accretion can reheat the white dwarf. If the accreted material ignites degenerately, the resultant nuclear explosion produces a nova and ejects all of the accreted material. But if this material burns non-degenerately, the white dwarf will gain mass. When the mass of the white dwarf exceeds the Chandrasekhar stability limit, it will begin to collapse.

Two possible fates await this collapsing white dwarf. If the collapsing core can achieve high enough temperatures, nuclear burning will begin and the stellar pressure will increase. This nuclear burning can drive nature's largest nuclear bomb, producing type Ia supernovae. However, neutrino emission from electron capture (e.g., URCA processes) can damp this burning until the core has collapsed too deeply into its gravitational well for nuclear burning to turn around the collapse. The result of this collapse is the formation of a neutron star. Electron capture will dominate if the density at which nuclear ignition occurs exceeds a critical density $\rho_{\text {crit }}$. For C-O white dwarfs, $\rho_{\text {crit }}$ is in the range $6 \times 10^{9}-10^{10} \mathrm{~g} \mathrm{~cm}^{-3}$ [32]. For O-Ne-Mg white dwarfs, electron capture may be stronger than nuclear burning under most conditions (if a Rayleigh-Taylor instability does not produce a turbulent burn front) $[187,127]$. The collapse of an O-Ne-Mg white dwarf begins when its central density reaches $4 \times 10^{9} \mathrm{~g} \mathrm{~cm}^{-3}$. (For more details about the conditions under which AIC occurs, see [187, 127, 32, 31, 156].) The dynamics of the collapse itself are somewhat similar to the dynamics of core collapse SNe: the collapse proceeds until the core reaches nuclear densities, the core then bounces and sends out a bounce shock that stalls when it becomes optically thin to neutrinos and loses its thermal energy. However, there is very little envelope around this core to prevent an explosion and the shock can easily be revived to drive a low-mass explosion. Exactly how much matter is ejected depends upon the details of the collapse calculation (compare [114, 263, 82]). Less than $10^{-1} M_{\odot}$ will likely be ejected by the star (due to the bounce itself or due to neutrino absorption/wind mechanisms) [82].

\subsection{Formation rate}

The AIC occurrence rate is difficult to determine for a number of reasons. These include incomplete understanding of binary star evolution and determining how much matter is truly accreted onto the white dwarf versus the amount that is ejected through novae [48, 137, 234]. Another uncertainty is whether the collapse of an accreting Chandrasekhar mass white dwarf results in a supernovae explosion or a complete AIC (with accompanying neutron star formation). Figure 1 shows one estimate of the region in the space of initial white dwarf mass and accretion rate that produces AICs. New results continue to alter the dividing lines between these fates [234].

The AIC rate can be indirectly inferred from the observed amount of rare, neutron rich isotopes present in the Galaxy. These isotopes (formed via electron capture) are present in the portion $\left(\sim 0.1 M_{\odot}\right)$ of the outer envelope ejected by the star during an AIC. The exact yield is sensitive to the neutron fraction in this ejecta, which depends both on the neutrino transport and the electron capture rates, but if all of these isotopes present in the Galaxy are assumed to have originated in AICs, an upper limit of $\sim 10^{-5} \mathrm{yr}^{-1}$ can be set for the Galactic AIC rate [82].

Binary population synthesis analysis can be used to determine which accreting white dwarfs will undergo AIC. The results of Yungelson and Livio [268] predict that the galactic AIC rate is between $8 \times 10^{-7}$ and $8 \times 10^{-5} \mathrm{yr}^{-1}$. Thus, a reasonable occurrence rate can be found for an observation distance of $100 \mathrm{Mpc}$. 


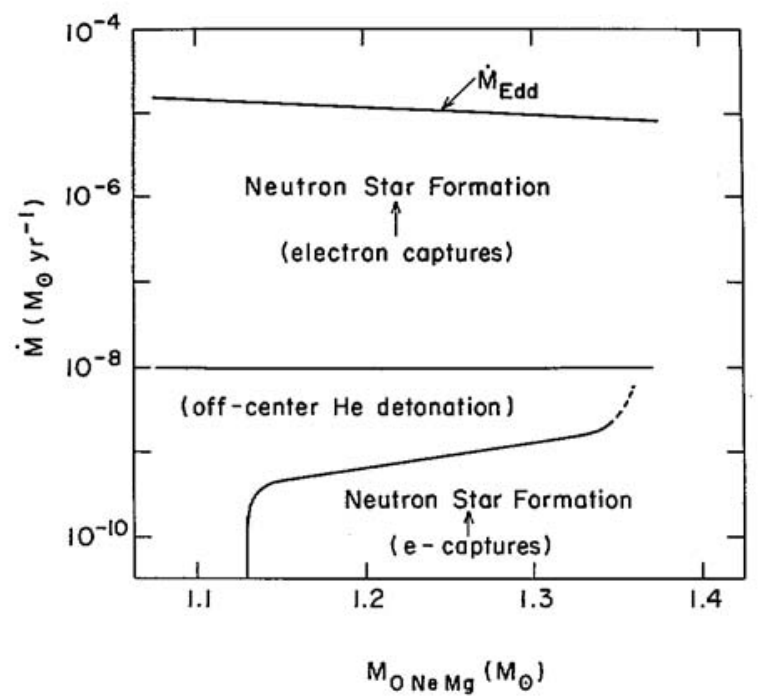

Figure 1: The final fate of accretion $O M g N e$ white dwarfs as a function of the initial white dwarf mass and the accretion rate onto the white dwarf. (Figure 3 of [187]; used with permission.)

\subsection{GW emission mechanisms}

During AIC, emission of GWs will occur if the infall of matter is aspherical. The convective time is likely to be brief, so it is unlikely that post-bounce convection will produce GWs. However, GWs will also be produced if the collapsing star or neutron star remnant develops rotational or pulsational instabilities [226, 227, 235, 252, 254]. These include global rotational mode, $r$-mode, $f$-mode, and fragmentation instabilities.

Global rotational instabilities in fluids arise from non-axisymmetric modes $e^{ \pm i m \phi}$, where $m=2$ is known as the "bar-mode" [239, 6]. It is convenient to parameterize a system's susceptibility to these modes by the stability parameter $\beta=T_{\text {rot }} /|W|$. Here, $T_{\text {rot }}$ is the rotational kinetic energy and $W$ is the gravitational potential energy. Dynamical rotational instabilities, driven by Newtonian hydrodynamics and gravity, develop on the order of the rotation period of the object. For the uniform-density, incompressible, uniformly rotating MacLaurin spheroids, the dynamical bar-mode instability sets in at $\beta_{\mathrm{d}} \approx 0.27$. For differentially rotating fluids with a polytropic equation of state, numerical simulations have determined that the stability limit $\beta_{\mathrm{d}} \approx$ 0.27 is valid for initial angular momentum distributions that are similar to those of MacLaurin spheroids $[221,63,162,125,192,118,248]$. If the object has an off-center density maximum, $\beta_{\mathrm{d}}$ could be as low as $0.10[247,260,192,49]$. General relativity may enhance the dynamical bar-mode instability by slightly reducing $\beta_{\mathrm{d}}[223,209]$. Secular rotational instabilities are driven by dissipative processes such as gravitational radiation reaction and viscosity. When this type of instability arises, it develops on the timescale of the relevant dissipative mechanism, which can be much longer than the rotation period (e.g., [225]. The secular bar-mode instability limit for MacLaurin spheroids is $\beta_{\mathrm{s}} \approx 0.14$.

In an attempt to reduce these high rotation requirements, there has been increasing work studying bar-mode instabilities driven by dynamical sheer in differentially rotating neutron stars. Sheer instabilities excite the co-rotating $f$-mode. If viscous forces don't damp this instability altogether, it is possible that this instability can occur for $\beta_{\mathrm{d}}$-values as low as $\sim 0.01$ for stars with a large degree of differential rotation.

Living Reviews in Relativity

http: //www. livingreviews. org/Irr-2003-2 
In rotating stars, gravitational radiation reaction drives the $r$-modes toward unstable growth $[5$, 77]. In hot, rapidly rotating neutron stars, this instability may not be suppressed by internal dissipative mechanisms (such as viscosity and magnetic fields) [152]. If not limited, the dimensionless amplitude $\alpha$ of the dominant $(m=2) r$-mode will grow to order unity within ten minutes of the formation of a neutron star rotating with a millisecond period. The emitted GWs carry away angular momentum, and will cause the newly formed neutron star to spin down over time. The spindown timescale and the strength of the GWs themselves are directly dependent on the maximum value $\alpha_{\max }$ to which the amplitude is allowed to grow $[153,154]$. Originally, it was thought that $\alpha_{\max } \sim 1$. Later work indicated that $\alpha_{\max }$ may be $\geq 3[153,236,213,154]$. Some research suggests that magnetic fields, hyperon cooling, and hyperon bulk viscosity may limit the growth of the $r$-mode instability, even in nascent neutron stars $[136,135,201,202,154,151,102,6]$ (significant uncertainties remain regarding the efficacy of these dissipative mechanisms). Furthermore, a study of a simple barotropic neutron star model by Arras et al. [9] suggests that multimode couplings could limit $\alpha_{\max }$ to values $\ll 1$. If $\alpha_{\max }$ is indeed $\ll 1$ (see also [97]), GW emission from $r$-modes in collapsed remnants is likely undetectable. For the sake of completeness, an analysis of GW emission from $r$-modes (which assumes $\alpha_{\max } \sim 1$ ) is presented in the remainder of this paper. However, because it is quite doubtful that $\alpha_{\max }$ is sizeable, $r$-mode sources are omitted from figures comparing source strengths and detector sensitivities and from discussions of likely detectable sources in the concluding section.

There is some numerical evidence that a collapsing star may fragment into two or more orbiting clumps [96]. If this does indeed occur, the orbiting fragments would be a strong GW source.

\subsection{Numerical predictions of GW emission}

\subsubsection{Full collapse simulations}

The accretion-induced collapse of white dwarfs has been simulated by a number of groups [14, 166, 263, 82]. The majority of these simulations have been Newtonian and have focused on mass ejection and neutrino and $\gamma$-ray emission during the collapse and its aftermath (note that neglecting relativistic effects likely introduces an error of order $(v / c)^{2} \sim 10 \%$ for the neutron star remnants of AIC; see below). The most sophisticated are those carried out by Fryer et al. [82], as they include realistic equations of state, neutrino transport, and rotating progenitors.

As a part of their general evaluation of upper limits to GW emission from gravitational collapse, Fryer, Holz, and Hughes (hereafter, FHH) [86] examined an AIC simulation (model 3) of Fryer et al. [82]. FHH used both numerical and analytical techniques to estimate the peak amplitude $h_{\mathrm{pk}}$, energy $E_{\mathrm{GW}}$, and frequency $f_{\mathrm{GW}}$ of the gravitational radiation emitted during the collapse simulations they studied.

For direct numerical computation of the GWs emitted in these simulations, FHH used the quadrupole approximation, valid for nearly Newtonian sources [169]. This approximation is standardly used to compute the GW emission in Newtonian simulations. The reduced or traceless quadrupole moment of the source can be expressed as

$$
\mathbf{f}_{i j}=\int \rho\left(x_{i} x_{j}-\frac{1}{3} \delta_{i j} r^{2}\right) d^{3} r
$$

where $i, j=1,2,3$ are spatial indices and $r=\sqrt{x^{2}+y^{2}+z^{2}}$ is the distance to the source. The two polarizations of the gravitational wave field, $h_{+}$and $h_{\times}$, can be computed in terms of $\dddot{\mathrm{f}}_{i j}$, where an overdot indicates a time derivative $d / d t$. The energy $E_{\mathrm{GW}}$ is a function of $\dddot{\mathfrak{f}}_{i j}$. Equation (1) can be used to calculate $\boldsymbol{\Psi}_{i j}$ from the results of a numerical simulation by direct summation over the computational grid. Numerical time derivatives of $\mathrm{I}_{i j}$ can then be taken to compute $h_{+}, h_{\times}$, and $E_{\mathrm{GW}}$. However, successive application of numerical derivatives generally introduces artificial 
noise. Methods for computing $\ddot{\mathfrak{f}}_{i j}$ and $\dddot{\mathrm{f}}_{i j}$ without taking numerical time derivatives have been developed [74, 25, 170]. These methods recast the time derivatives of $\boldsymbol{f}_{i j}$ as spatial derivatives of hydrodynamic quantities computed in the collapse simulation (including the density, velocities, and gravitational potential). Thus, instantaneous values for $\ddot{\mathfrak{f}}_{i j}$ and $\dddot{\mathrm{f}}_{i j}$ can be computed on a single numerical time slice (see $[170,271]$ for details). Note that FHH define $h_{\mathrm{pk}}$ as the maximum value of the rms strain $h=\sqrt{\left\langle h_{+}^{2}+h_{\times}^{2}\right\rangle}$, where angular brackets indicate that averages have been taken over both wavelength and viewing angle on the sky.

Errors resulting from the neglect of general relativistic effects (in collapse evolutions as a whole and in GW emission estimations like the quadrupole approximation) are of order $(v / c)^{2}$. These errors are typically $\sim 10 \%$ for neutron star remnants of AIC, $<30 \%$ for neutron star remnants of massive stellar collapse, and $>30 \%$ for black hole remnants. Neglect of general relativity in rotational collapse studies is of special concern because relativistic effects counteract the stabilizing effects of rotation (see Section 3.4).

Because the code used in the collapse simulations examined by FHH [82] was axisymmetric, their use of the numerical quadrupole approximation discussed above does not account for GW emission that may occur due to non-axisymmetric mass flow. The GWs computed directly from their simulations come only from polar oscillations (which are significant when the mass flow during collapse [or explosion] is largely aspherical).

In order to predict the GW emission produced by non-axisymmetric instabilities, FHH employed rough analytical estimates. The expressions they used to approximate the rms strain $h$ and the power $P=d E_{\mathrm{GW}} / d t$ of the GWs emitted by a star that has encountered the bar-mode instability are

and

$$
h_{\mathrm{bar}}=\sqrt{\frac{32}{45}} \frac{G}{c^{4}} \frac{m r^{2} \omega^{2}}{d}
$$

$$
P_{\text {bar }}=\frac{32}{45} \frac{G}{c^{5}} m^{2} r^{4} \omega^{6} .
$$

Here $m, 2 r$, and $\omega$ are the mass, length, and angular frequency of the bar and $d$ is the distance to the source. FHH vary the mass $m$ assumed to be enclosed by the bar (which has a corresponding length $2 r$ ) and compute the characteristics of the GW emission as a function of this enclosed mass. For simplicity, FHH assumed that a fragmentation instability will cause a star to break into two clumps (although more clumps could certainly be produced). Their estimates for the rms strain and power radiated by the orbiting binary fragments are

$$
h_{\text {bin }}=\sqrt{\frac{128}{5}} \frac{G}{c^{4}} \frac{m r^{2} \omega^{2}}{d}
$$

and

$$
P_{\mathrm{bin}}=\frac{128}{5} \frac{G}{c^{5}} m^{2} r^{4} \omega^{6}
$$

Here $m$ is the mass of a single fragment, $2 r$ is the separation of the fragments, and $\omega$ is their orbital frequency.

For their computation of the GWs radiated via $r$-modes, FHH used the method of Ho and Lai [115] (which assumes $\alpha_{\max }=1$ ) and calculated only the emission from the dominant $m=2$ mode. This approach is detailed in FHH. If the neutron star mass and initial radius are taken to be $1.4 M_{\odot}$ and $12.53 \mathrm{~km}$, respectively, the resulting formula for the average GW strain is

$$
h(t)=1.8 \times 10^{-24} \alpha\left(\frac{\nu_{\mathrm{s}}}{1 \mathrm{kHz}}\right)\left(\frac{20 \mathrm{Mpc}}{d}\right),
$$

where $\alpha$ is the mode amplitude and $\nu_{\mathrm{s}}$ is the spin frequency.

Living Reviews in Relativity

http://www. livingreviews.org/lrr-2003-2 
FHH's numerical quadrupole estimate of the GWs from polar oscillations in the AIC simulation of Fryer et al. [82] predicts a peak dimensionless amplitude $h_{\mathrm{pk}}=5.9 \times 10^{-24}$ (for $d=100 \mathrm{Mpc}$ ). The energy $E_{\mathrm{GW}}=3 \times 10^{45} \mathrm{erg}$ is emitted at a frequency of $f_{\mathrm{GW}} \approx 50 \mathrm{~Hz}$. This amplitude is about an order of magnitude too small to be observed by the advanced LIGO-II detector. The sensitivity curve for the broadband configuration of the LIGO-II detector is shown in Figure 2 (see Appendix A of [86] for details on the computation of this curve). Note that the characteristic strain $h$ is plotted along the vertical axis in Figure 2 (and in LISA's sensitivity curve, shown in Figure 18). For burst sources, $h=h_{\mathrm{pk}}$. For sources that persist for $N$ cycles, $h=\sqrt{N} h_{\mathrm{pk}}$.

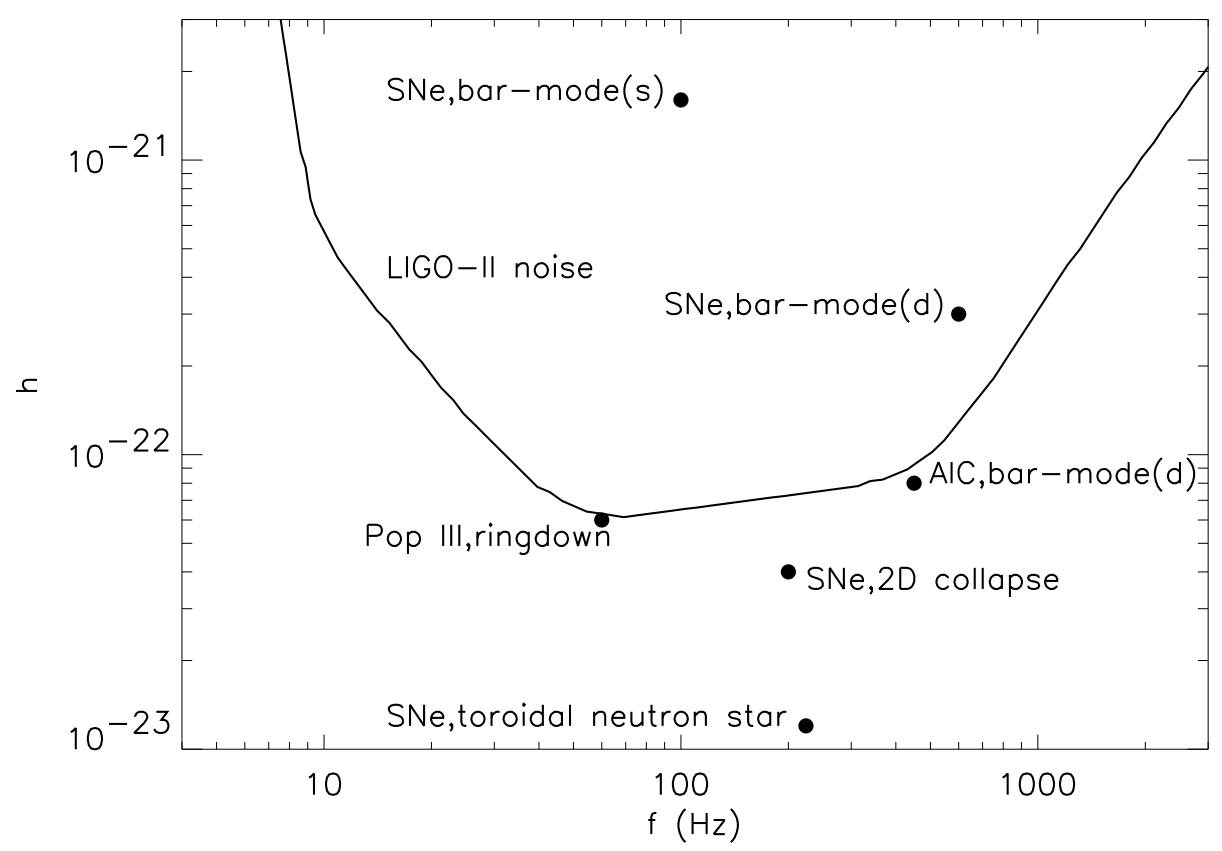

Figure 2: A comparison between the GW amplitude $h(f)$ for various sources and the LIGO-II sensitivity curve. See the text for details regarding the computations of $h$. The AIC sources are assumed to be located at a distance of $100 \mathrm{Mpc}$; the SNe sources at $10 \mathrm{Mpc}$; and the Population III sources at a luminosity distance of $\sim 50 \mathrm{Gpc}$. Secular bar-mode sources are identified with an (s), dynamical bar-modes with a (d).

The simulation of Fryer et al. [82] considered by FHH does produce an object with an off-center density maximum. However, because the maximum $\beta$ reached in the AIC simulation of Fryer et al. was $<0.06$ (and because the degree of differential rotation present in the remnant was low), the collapsing object is not likely to encounter dynamical rotational instabilities. The choice of initial angular momentum $J$ for the progenitor in an AIC simulation can affect this outcome. Fryer et al.'s choice of $J=10^{49} \mathrm{~g} \mathrm{~cm}^{2} \mathrm{~s}^{-1}$ is such that the period of the white dwarf progenitor is $10 \mathrm{~s}$ less than the shortest observed period $30 \mathrm{~s}$ of a cataclysmic variable white dwarf [139]. Higher angular momenta are certainly possible. If higher values of $J$ exist in accreting white dwarfs, bar-mode instabilities may be more likely to occur (see the discussion of work by Liu and Lindblom below).

According to $\mathrm{FHH}$, the remnant of this AIC simulation will be susceptible to $r$-mode growth. Assuming $\alpha_{\max } \sim 1$ (which is likely not physical; see Section 2.3), they predict $E_{\mathrm{GW}}>10^{52} \mathrm{erg}$. FHH compute $h\left(f_{\mathrm{GW}}\right)$ for coherent observation of the neutron star as it spins down over the course of a year. For a neutron star located at a distance of $100 \mathrm{Mpc}$, this track is always below the LIGOII noise curve. The point on this track with the maximum $h$, which corresponds to the beginning 
of $r$-mode evolution, is shown in Figure 2. The track moves down and to the left (i.e., $h$ and $f$ decrease) in this figure as the $r$-mode evolution continues.

\subsubsection{Analyses of equilibrium representations of collapse}

In addition to full hydrodynamics collapse simulations, many studies of gravitational collapse have used hydrostatic equilibrium models to represent stars at various stages in the collapse process. Some investigators use sequences of equilibrium models to represent snapshots of the phases of collapse (e.g., [16, 185, 156]). Others use individual equilibrium models as initial conditions for hydrodynamical simulations (e.g., [231, 192, 184, 49]). Such simulations represent the approximate evolution of a model beginning at some intermediate phase during collapse or the evolution of a collapsed remnant. These studies do not typically follow the intricate details of the collapse itself. Instead, their goals include determining the stability of models against the development of nonaxisymmetric modes and estimation of the characteristics of any resulting GW emission.

Liu and Lindblom $[156,155]$ have applied this equilibrium approach to AIC. Their investigation began with a study of equilibrium models built to represent neutron stars formed from AIC [156]. These neutron star models were created via a two-step process, using a Newtonian version of Hachisu's self-consistent field method [98]. Hachisu's method ensures that the forces due to the centrifugal and gravitational potentials and the pressure are in balance in the equilibrium configuration.

Liu and Lindblom's process of building the nascent neutron stars began with the construction of rapidly rotating, pre-collapse white dwarf models. Their Models I and II are C-O white dwarfs with central densities $\rho_{\mathrm{c}}=10^{10}$ and $6 \times 10^{9} \mathrm{~g} \mathrm{~cm}^{-3}$, respectively (recall this is the range of densities for which AIC is likely for C-O white dwarfs). Their Model III is an O-Ne-Mg white dwarf that has $\rho_{\mathrm{c}}=4 \times 10^{9} \mathrm{~g} \mathrm{~cm}^{-3}$ (recall this is the density at which collapse is induced by electron capture). All three models are uniformly rotating, with the maximum allowed angular velocities. The models' values of total angular momentum are roughly 3-4 times that of Fryer et al.'s AIC progenitor Model 3 [82]. The realistic equation of state used to construct the white dwarfs is a Coulomb corrected, zero temperature, degenerate gas equation of state [210, 52].

In the second step of their process, Liu and Lindblom [156] built equilibrium models of the collapsed neutron stars themselves. The mass, total angular momentum, and specific angular momentum distribution of each neutron star remnant is identical to that of its white dwarf progenitor (see Section 3 of [156] for justification of the specific angular momentum conservation assumption). These models were built with two different realistic neutron star equations of state.

Liu and Lindblom's cold neutron star remnants had values of the stability parameter $\beta$ ranging from 0.23-0.26. It is interesting to compare these results with those of Zwerger and Müller [271]. Zwerger and Müller performed axisymmetric hydrodynamics simulations of stars with polytropic equations of state $\left(P \propto \rho^{\Gamma}\right)$. Their initial models were $\Gamma=4 / 3$ polytropes, representative of massive white dwarfs. All of their models started with $\rho_{\mathrm{c}}=10^{10} \mathrm{~g} \mathrm{~cm}^{-3}$. Their model that was closest to being in uniform rotation (A1B3) had $22 \%$ less total angular momentum than Liu and Lindblom's Model I. The collapse simulations of Zwerger and Müller that started with model A1B3 all resulted in remnants with values of $\beta<0.07$. Comparison of the results of these two studies could indicate that the equation of state may play a significant role in determining the structure of collapsed remnants. Or it could suggest that the assumptions employed in the simplified investigation of Liu and Lindblom are not fully appropriate. Zwerger and Müller's work will be discussed in much more detail in Section 3, as it was performed in the context of core collapse supernovae.

In a continuation of the work of Liu and Lindblom, Liu [155] used linearized hydrodynamics to perform a stability analysis of the cold neutron star AIC remnants of Liu and Lindblom [156]. He found that only the remnant of the O-Ne-Mg white dwarf (Liu and Lindblom's Model III)

Living Reviews in Relativity

http://www. livingreviews . org//rr-2003-2 
developed the dynamical bar-mode $(m=2)$ instability. This model had an initial $\beta=0.26$. Note that the $m=1$ mode, observed by others to be the dominant mode in unstable models with values of $\beta$ much lower than 0.27 [247, 260, 192, 49], did not grow in his simulation. Because Liu and Lindblom's Models I and II had lower values of $\beta$, Liu identified the onset of instability for neutron stars formed via AIC as $\beta_{\mathrm{d}} \approx 0.25$.

Liu estimated the peak amplitude of the GWs emitted by the Model III remnant to be $h_{\mathrm{pk}} \approx$ $1.4 \times 10^{-24}$ and the LIGO-II signal-to-noise ratio (for a persistent signal like that seen in the work of [184] and [34]) to be $S / N \leq 3$ (for $f_{\mathrm{GW}} \approx 450 \mathrm{~Hz}$ ). These values are for a source located at $100 \mathrm{Mpc}$. He also predicted that the timescale for gravitational radiation to carry away enough angular momentum to eliminate the bar-mode is $\tau_{\mathrm{GW}} \sim 7 \mathrm{~s}\left(\sim 3 \times 10^{3}\right.$ cycles $)$. Thus, $h \sim 8 \times 10^{-23}$. (Note that this value for $h$ is merely an upper limit as it assumes that the amplitude and frequency of the GWs do not change over the $7 \mathrm{~s}$ during which they are emitted. Of course, they will change as angular momentum is carried away from the object via GW emission.) Such a signal may be marginally detectable with LIGO-II (see Figure 2). Details of the approximations on which these estimates are based can be found in [155].

Liu cautions that his results hold if the magnetic field of the proto-neutron star is $B \leq 10^{12} \mathrm{G}$. If the magnetic field is larger, then it may have time to suppress some of the neutron star's differential rotation before it cools. This would make bar formation less likely. Such a large field could only result if the white dwarf progenitor's $B$ field was $\geq 10^{8} \mathrm{G}$. Observation-based estimates suggest that about $25 \%$ of white dwarfs in interacting close binaries (cataclysmic variables) are magnetic and that the field strengths for these stars are $\sim 10^{7}-3 \times 10^{8} \mathrm{G}[256]$.

\subsection{Going further}

The AIC scenario is generally discussed in terms of the collapse of an accreting white dwarf to a neutron star. However, Shibata, Baumgarte, and Shapiro have examined the collapse of a rotating, supramassive neutron star to a black hole [224]. Such supramassive neutron stars (with masses greater than the maximum mass for a nonrotating neutron star) could be formed and pushed to collapse via accretion from a binary companion. They performed 3D, fully general relativistic hydrodynamics simulations of uniformly rotating neutron stars. Dynamical non-axisymmetric instabilities (such as the bar-mode) did not have time to grow in their simulations prior to black hole formation. Differentially rotating neutron star progenitors could have higher values of $\beta$ than the uniformly rotating models used in this study and may be susceptible to non-axisymmetric instabilities on a shorter timescale. 


\section{Collapse of Massive Stars}

\subsection{Collapse scenario}

Stars with mass greater than $\sim 8-10 M_{\odot}$ will undergo core collapse at the end of their thermonuclear burning life cycles. The gravitational energy released during the collapse is believed to be the power source behind a large subset of supernovae (for reviews, see [8, 23, 79, 81, 143]). Core collapse SNe include Types II and Ib/Ic. SNe Ib/Ic are distinguished from Type II SNe by the absence of hydrogen in their spectra. SNe Ib/Ic are thought to result from the collapse of the cores of massive stars that have lost their hydrogen envelopes (and possibly part of their helium envelopes) by stellar winds or by mass transfer. The SN Ib/Ic progenitors that lose their outer envelopes via stellar winds are known as Wolf-Rayet stars and have initial masses $\gtrsim 30 M_{\odot}[108,266]$; those that undergo mass loss via mass transfer in binaries can have progenitor masses as low as $12 M_{\odot}$ [194].

If the stellar mass is less than $\sim 20-25 M_{\odot}[78,89,86,108]$, it is believed that the star will produce a strong supernova, leaving behind a neutron star remnant. This section studies the gravitational waves produced during the stellar collapse and supernova explosion mechanism. Without mass loss, stars above $40-50 M_{\odot}$ are thought to collapse directly to black holes without producing a supernova explosion [78, 108]. If rotating, the collapsing stars may form an accretion disk around their black hole core. One of the leading models for long-duration gamma-ray bursts (the "collapsar" engine) argues that the energy extracted from the disk or the black hole spin can drive a relativistic jet [262]. We will discuss these collapsars and their resultant gravitational waves further in Section 4. However, if we include the effects of winds, these stars lose much of their mass through winds during their nuclear-burning lifetimes. Their fate will be closer to that of a $20 M_{\odot}$ and are likely to produce supernovae and, at least initially, neutron stars, not black holes [89, 108]. If the progenitor's mass is in the range $20-25 \lesssim M \lesssim 40-50 M_{\odot}$, the entire star is not ejected in the SN explosion. More than $2 M_{\odot}$ will fall back onto the nascent neutron star and lead to black hole formation. These objects also produce a variant of the collapsar engine for gamma-ray bursts (GRBs). Although the discussion of GW production from the collapse and supernova explosion phase will be discussed in this section, the GWs produced during the fallback and black hole accretion disk phase will be discussed in Section 4. Note that the limits on the progenitor masses quoted in this paragraph (especially the $40-50 M_{\odot}$ lower limit for direct black hole formation) are uncertain because the progenitor mass dependence of the neutrino explosion mechanism (see below) is unknown [103, 178].

The massive iron cores of SN II/Ib/Ic progenitors are supported by both thermal and electron degeneracy pressures. The density and temperature of such a core will eventually rise, due to the build up of matter consumed by thermonuclear burning, to the point where electron capture and photodissociation of nuclei begin. Dissociation lowers the photon and electron temperatures and thereby reduces the core's thermal support [71]. Electron capture reduces the electron degeneracy pressure. One or both of these processes will trigger the collapse of the core. The relative importance of dissociation and electron capture in instigating collapse is determined by the mass of the star [71]. The more massive the core, the bigger is the role played by dissociation.

Approximately $70 \%$ of the inner portion of the core collapses homologously and subsonically. The outer core collapses at supersonic speeds [71, 170]. The maximum velocity of the outer regions of the core reaches $\sim 7 \times 10^{4} \mathrm{~km} \mathrm{~s}^{-1}$. It takes just $1 \mathrm{~s}$ for an earth-sized core to collapse to a radius of $50 \mathrm{~km}[8]$.

The inward collapse of the core is halted by nuclear forces when its central density $\rho_{\mathrm{c}}$ is $2-10$ times the density of nuclear material $[13,12]$. The core overshoots its equilibrium position and bounces. A shock wave is formed when the supersonically infalling outer layers hit the rebounding inner core. If the inner core pushes the shock outwards with energy $E>10^{51}$ erg (supplied by the binding energy of the nascent neutron star), then the remainder of the star can be ejected

Living Reviews in Relativity

http://www. livingreviews.org/lrr-2003-2 
in about $20 \mathrm{~ms}$ [8]. This so-called "prompt explosion" mechanism has been succesful numerically only when a very soft supra-nuclear equation of state is used in conjunction with a relatively small core $\left(M \lesssim 1.35 M_{\odot}\right.$, derived from a very low mass progenitor) and a large portion of the collapse proceeds homologously $[23,173]$. Inclusion of general relativistic effects in collapse simulations can increase the success of the prompt mechanism in some cases [13, 237].

Both dissociation of nuclei and electron capture can reduce the ejection energy, causing the prompt mechanism to fail. The shock will then stall at a radius in the range $100-200 \mathrm{~km}$. Colgate and White [55] suggested that energy from neutrinos emitted by the collapsed core could revive the stalled shock. (See Burrows and Thompson [44] for a review of core collapse neutrino processes.) However, their simulations did not include the physics necessary to accurately model this "delayed explosion" mechanism. Wilson and collaborators were the first to perform collapse simulations with successful delayed ejections [30, 29, 257, 24, 259, 258]. However, their 1-dimensional simulations and those of others had difficulty producing energies high enough to match observations [53, 36, 131]. It was not until Herant and collaborators modelled the collapse and bounce phase with neutrinotransport in 2-dimensions that convection began to be accepted as a necessary puzzle piece in understanding the supernova explosion mechanism [112].

Observations of SN 1987a show that significant mixing occurred during this supernovae (see Arnett et al. [8] for a review). Such mixing can be attributed to nonradial motion resulting from fluid instabilities. Convective instabilities play a significant role in the current picture of the delayed explosion mechanism. The outer regions of the nascent neutron star are convectively unstable after the shock stalls (for an interval of 10-100 ms after bounce) due to the presence of negative lepton and energy gradients [173]. This has been confirmed by both 2D and 3D simulations $[112,42,132,173,167,78,83,128,130,197,91,85,26,39,92,80,212,253]$. Convective motion is more effective at transporting neutrinos out of the proto-neutron star than is diffusion. Less than $10 \%$ of the neutrinos emitted by the neutron star need to be absorbed and converted to kinetic energy for the shock to be revived [173]. The "hot bubble" region above the surface of the neutron star also has been shown to be convectively unstable [53, 23, 54, 91]. Janka and Müller have demonstrated that convection in this region only aids the explosion if the neutrino luminosity is in a narrow region [133]. Some simulations that include advanced neutrino transport methods have cast doubt on the ability of convection to ensure the success of the delayed explosion mechanism $[168,130,197,39]$ and this problem is far from solved. But progress not only in neutrino transport, but in understanding new features in the convection $[26,43,75]$ some including the effect of magnetic fields is leading to new ideas about the supernova mechanism [3]. We will discuss the effects of this new physics on the GW signal at the end of this section.

In addition to the mixing seen in SN 1987a, observations of (i) polarization in the spectra of several core collapse SNe, (ii) jets in the Cas A remnant, and (iii) kicks in neutron stars suggest that supernovae are inherently aspherical (see [8, 3, 116, 122, 121] and references therein). Note that these asphericities could originate in the central explosion mechanism itself and/or the mechanism(s) for energy transfer between the core and ejecta [130]. If due to the mechanism itself, these asymmetries may provide clues into the true engine behind supernova explosions. Already, the observations partly motivated the multi-dimensional studies of convection in the delayed explosion mechanism as well as work on magnetic field engines [17, 255, 3]. Observations have also driven the work on jets and collapsars 4. Höflich et al. [116] have argued that low velocity jets stalled inside SN envelopes can account for the observed asymmetries. Hungerford and collaborators have argued that the asymmetries required are not so extreme [122, 121], arguments that have now been confirmed [138]. This debate is crucial to our understanding of the supernova mechanism. If jets are required, magnetic field mechanisms are the likely source of the asymmetry. If jets are not required, the convective engine can produce asymmetries via a number of channels from low mode convection [26, 212, 43] to rotation(e.g.,[83]) to asymmetric collapse [41, 80]. Most GW emission calculations of stellar collapse have focused on the collapse of rotating, massive stars with rotation 
periods at least as high as those assumed by Fryer \& Heger [83], implicitly assuming that the asymmetries are driving by rotation.

\subsection{Formation rate}

Type II/Ib/Ic supernovae are observed to occur in only spiral and irregular galaxies. The most thorough computation of SN rates is that of Cappellaro et al. [46]. Their sample includes $137 \mathrm{SNe}$ from five different SN searches. They determined that the core collapse SN rate in the Galaxy is $6 \times 10^{-3}-1.6 \times 10^{-2} \mathrm{yr}^{-1}$. Thus, a reasonable occurrence rate can be found for an observation distance of $10 \mathrm{Mpc}$. (Note that a infrared survey estimates that the rate may actually be an order of magnitude higher [161].) What this rate might exclude are those stars that collapse to form neutron stars, but have sufficiently weak explosions that they eject very little nickel and hence are not very bright. Including these systems as well will increase the total number of stellar collapses initially into neutron stars by less than 5-40\% [89]. However, it does include those stars that collapse to black holes directly and form GRBs (producing supernova-like outbursts). We will discuss the GWs from such collapses in Section 4. Excluding them from this sample will not change the above numbers.

\subsection{GW emission mechanisms}

Gravitational radiation will be emitted during the collapse/explosion of a core collapse SN due to the star's changing quadrupole moment. A rough description of the possible evolution of the quadrupole moment is given in the remainder of this paragraph. During the first 100-250 ms of the collapse, as the core contracts and flattens, the magnitude of the quadrupole moment $\boldsymbol{\Psi}_{i j}$ will increase. The contraction speeds up over the next $20 \mathrm{~ms}$ and the density distribution becomes a centrally condensed torus [170]. In this phase the core's shrinking size dominates its increasing deformation and the magnitude of $\boldsymbol{f}_{i j}$ decreases. As the core bounces, $\mathbf{f}_{i j}$ changes rapidly due to the deceleration and rebound. If the bounce occurs because of nuclear pressure, its timescale will be $<1 \mathrm{~ms}$. If centrifugal forces play a role in halting the collapse, the bounce can last up to several ms [170]. The magnitude of $\mathbf{f}_{i j}$ will increase due to the core's expansion after bounce. As the resulting shock moves outwards, the unshocked portion of the core will undergo oscillations, causing $\mathrm{f}_{i j}$ to oscillate as well. The shape of the core, the depth of the bounce, the bounce timescale, and the rotational energy of the core all strongly affect the GW emission. For further details see $[71,170]$.

Convectively driven inhomogeneities in the density distribution of the outer regions of the nascent neutron star and anisotropic neutrino emission are other sources of GW emission during the collapse/explosion (see [41, 176, 80, 158] for reviews).

As discussed in the case of AIC, global rotational instabilities (such as the $m=2$ bar-mode) may develop during the collapse itself or in a neutron star remnant. A neutron star remnant will likely also be susceptible to the radiation reaction driven $r$-modes. Both of these types of instabilities will emit GWs, as will a fragmentation instability if one occurs. See Section 2.3 for further details regarding these instabilities.

\subsection{Numerical predictions of GW emission}

\subsubsection{Historical investigations}

The collapse of the progenitors of core collapse supernovae has been investigated as a source of gravitational radiation for more than three decades. In an early study published in 1971, Ruffini and Wheeler [203] identified mechanisms related to core collapse that could produce GWs and provided order-of-magnitude estimates of the characteristics of such emission.

Living Reviews in Relativity

http://www. livingreviews . org//rr-2003-2 
Quantitative computations of GW emission during the infall phase of collapse were performed by Thuan and Ostriker [245] and Epstein and Wagoner [66, 65], who simulated the collapse of oblate dust spheroids. Thuan and Ostriker used Newtonian gravity and computed the emitted radiation in the quadrupole approximation. Epstein and Wagoner discovered that post-Newtonian effects prolonged the collapse and thus lowered the GW luminosity. Subsequently, Novikov [188] and Shapiro and Saenz $[218,204]$ included internal pressure in their collapse simulations and were thus able to examine the GWs emitted as collapsing cores bounced at nuclear densities. The quadrupole GWs from the ringdown of the collapse remnant were initially investigated by the perturbation study of Turner and Wagoner [249] and later by Saenz and Shapiro [205, 206].

Müller [171] calculated the quadrupole GW emission from 2D axisymmetric collapse based on the Newtonian simulations of Müller and Hillebrandt [174] (these simulations used a realistic equation of state and included differential rotation). He found that differential rotation enhanced the efficiency of the GW emission.

Stark and Piran $[233,193]$ were the first to compute the GW emission from fully relativistic collapse simulations, using the ground-breaking formalism of Bardeen and Piran [11]. They followed the (pressure-cut induced) collapse of rotating polytropes in 2D. Their work focused in part on the conditions for black hole formation and the nature of the resulting ringdown waveform, which they found could be described by the quasi-normal modes of a rotating black hole. In each of their simulations, less than $1 \%$ of the gravitational mass was converted to GW energy.

Seidel and collaborators also studied the effects of general relativity on the GW emission during collapse and bounce $[215,216]$. They employed a perturbative approach, valid only in the slowly rotating regime.

The gravitational radiation from non-axisymmetric collapse was investigated by Detweiler and Lindblom, who used a sequence of non-axisymmetric ellipsoids to represent the collapse evolution [57]. They found that the radiation from their analysis of non-axisymmetric collapse was emitted over a more narrow range of frequency than in previous studies of axisymmetric collapse.

For further discussion of the first two decades of study of the GW emission from stellar collapse see [72]. In the remainder of Section 3.4, more recent investigations will be discussed.

\subsubsection{Axisymmetric simulations}

The core collapse simulations of Mönchmeyer et al. began with better iron core models and a more realistic microphysical treatment (including a realistic equation of state, electron capture, and a simple neutrino transport scheme) than any previous study of GW emission from axisymmetric stellar core collapse [170]. The shortcomings of their investigation included initial models that were not in rotational equilibrium, an equation of state that was somewhat stiff in the subnuclear regime, and the use of Newtonian gravity. Each of their four models had a different initial angular momentum profile. The rotational energies of the models ranged from $0.1-0.45$ of the maximum possible rotational energy.

The collapses of three of the four models of Mönchmeyer et al. were halted by centrifugal forces at subnuclear densities. This type of low $\rho_{\mathrm{c}}$ bounce had been predicted by Shapiro and Lightman [219] and Tohline [246] (in the context of the "fizzler" scenario for failed supernovae; see also $[105,106,124]$ ), and had been observed in earlier collapse simulations [175, 238]. Mönchmeyer and collaborators found that a bounce caused by centrifugal forces would last for several ms, whereas a bounce at nuclear densities would occur in $<1 \mathrm{~ms}$. They also determined that a subnuclear bounce produced larger amplitude oscillations in density and radius, with larger oscillation periods, than a bounce initiated by nuclear forces alone. They pointed out that these differences in timescale and oscillatory behavior should affect the GW signal. Therefore, the GW emission could indicate whether the bounce was a result of centrifugal or nuclear forces.

Mönchmeyer et al. identified two different types of waveforms in their models (computed using 
the numerical quadrupole approximation discussed in Section 2.4). The waveforms they categorized as Type I (similar to those observed in previous collapse simulations [171, 74]) are distinguished by a large amplitude peak at bounce and subsequent damped ringdown oscillations. They noted that Type I signals were produced by cores that bounced at nuclear densities (or bounced at subnuclear densities if the cores had small ratios of radial kinetic to rotational kinetic energies). The quadrupole gravitational wave amplitude $A_{20}^{\mathrm{E} 2}$ for a Type I waveform is shown in Figure 3 (see [241, 271] for expressions relating $A_{20}^{\mathrm{E} 2}$ to $h$ ). The waveforms identified as Type II exhibit several maxima, which result from multiple bounces (see Figure 4 for an example of a Type II waveform). Note that the waveforms displayed in Figures 3 and 4 are from the study of Zwerger and Müller [271], discussed below.

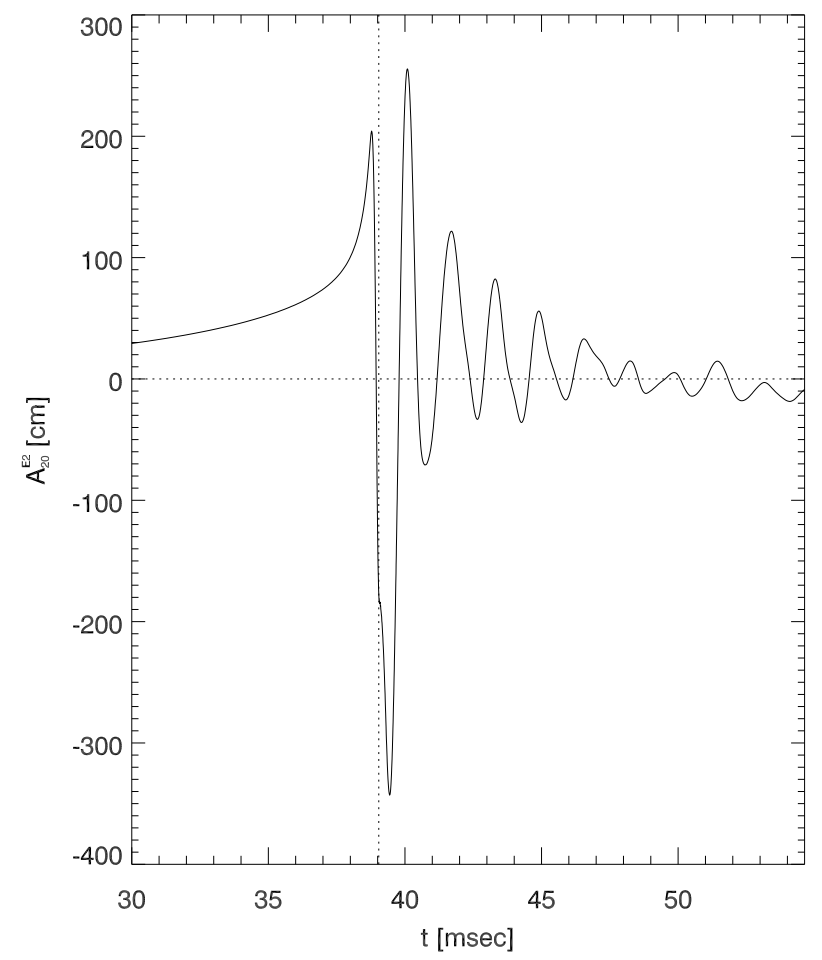

Figure 3: Type I waveform (quadrupole amplitude $A_{20}^{\mathrm{E} 2}$ as a function of time) from one of Zwerger and Müller's [271] simulations of a collapsing polytrope. The vertical dotted line marks the time at which the first bounce occurred. (Figure 5d of [271]; used with permission.)

The model of Mönchmeyer et al. that bounced due to nuclear forces had the highest GW amplitude of all of their models, $h_{\mathrm{pk}} \sim 10^{-23}$ for a source distance $d=10 \mathrm{Mpc}$, and the largest emitted energy $E_{\mathrm{GW}} \sim 10^{47} \mathrm{erg}$. The accompanying power spectrum peaked in the frequency range $5 \times 10^{2}-10^{3} \mathrm{~Hz}$.

The most extensive Newtonian survey of the parameter space of axisymmetric, rotational core collapse is that of Zwerger and Müller [271]. They simulated the collapse of 78 initial models with varying amounts of rotational kinetic energy (reflected in the initial value of the stability parameter $\beta_{\mathrm{i}}$ ), differential rotation, and equation of state stiffness. In order to make this large survey tractable, they used a simplified equation of state and did not explicitly account for electron capture or neutrino transport. Their initial models were constructed in rotational equilibrium via the method of Eriguchi and Müller [67]. The models had a polytropic equation of state, with initial adiabatic index $\Gamma_{\mathrm{i}}=4 / 3$. Collapse was induced by reducing the adiabatic index to a value

Living Reviews in Relativity

http://www. livingreviews.org/lrr-2003-2 


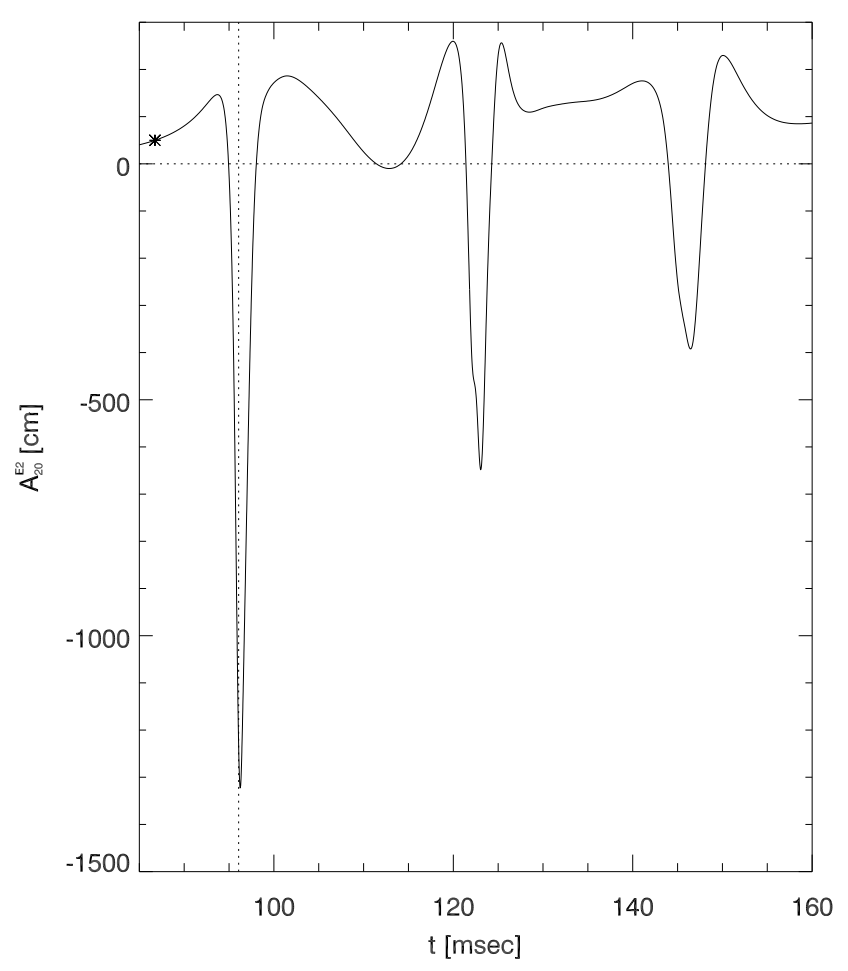

Figure 4: Type II waveform (quadrupole amplitude $A_{20}^{\mathrm{E} 2}$ as a function of time) from one of Zwerger and Müller's [271] simulations of a collapsing polytrope. The vertical dotted line marks the time at which bounce occurred. (Figure 5 a of [271]; used with permission.) 
$\Gamma_{\mathrm{r}}$ in the range 1.28-1.325. The equation of state used during the collapse evolution had both polytropic and thermal contributions (note that simulations using more sophisticated equations of state get similar results [144]).

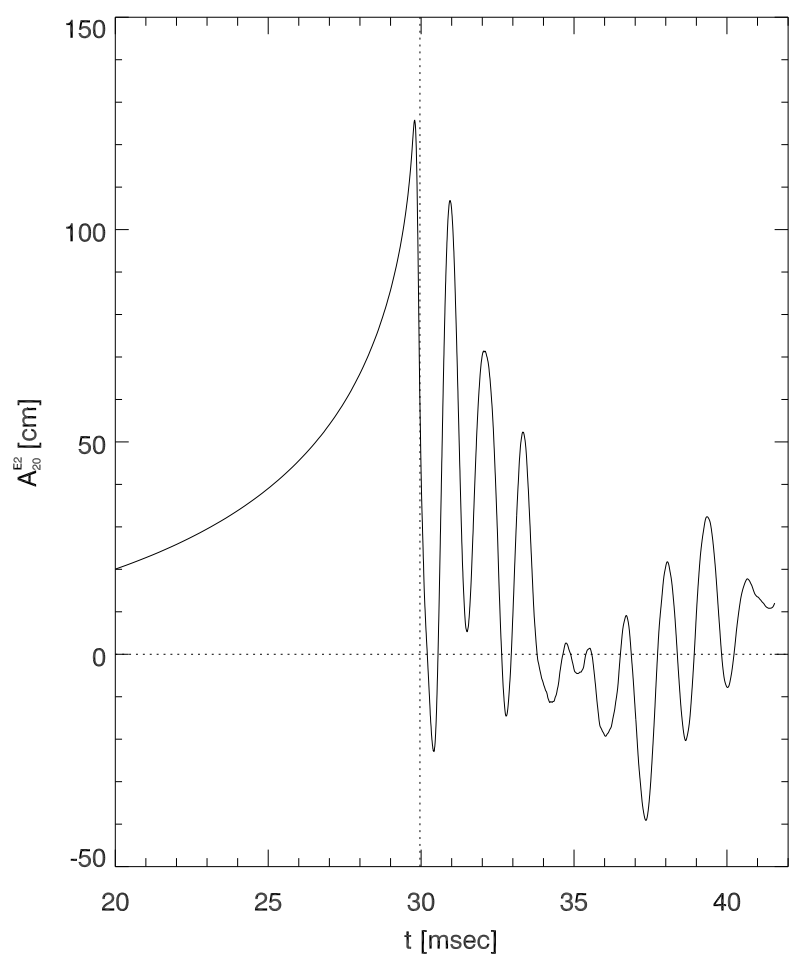

Figure 5: Type III waveform (quadrupole amplitude $A_{20}^{\mathrm{E} 2}$ as a function of time) from one of Zwerger and Müller's [271] simulations of a collapsing polytrope. The vertical dotted line marks the time at which bounce occurred. (Figure 5e of [271]; used with permission).

The major result of Zwerger and Müller's investigation was that the signal type of the emitted gravitational waveform in their runs was determined by the stiffness of the equation of state of the collapsing core (i.e., the value of $\Gamma_{\mathrm{r}}$ ). In their simulations, Type I signals (as labelled by Mönchmeyer et al. [170]) were produced by models with relatively soft equations of state, $\Gamma_{\mathrm{r}} \lesssim 1.31$. Type II signals were produced by the models with stiffer equations of state, $\Gamma_{\mathrm{r}} \gtrsim 1.32$. They found a smooth transition between these signal types if $\Gamma_{\mathrm{r}}$ was increased while all other parameters were held fixed. They also observed another class of signal, Type III, for their models with the lowest $\Gamma_{\mathrm{r}}(=1.28)$. Type III waveforms have a large positive peak just prior to bounce, a smaller negative peak just after bounce, and smaller subsequent oscillations with very short periods (see Figure 5). Type III signals were not observed in the evolution of strongly differentially rotating $\Gamma_{\mathrm{r}}=1.28$ models and were also not seen in subsequent investigations $[144,190]$. Their waveforms were computed with the same technique used in [170].

In contrast to the results of Mönchmeyer et al. [170], in Zwerger and Müller's investigation the value of $\rho_{\mathrm{c}}$ at bounce did not determine the signal type. Instead, the only effect on the waveform due to $\rho_{\mathrm{c}}$ was a decrease in $h_{\mathrm{pk}}$ in models that bounced at subnuclear densities. The effect of the initial value of $\beta_{\mathrm{i}}$ on $h_{\mathrm{pk}}$ was non-monotonic. For models with $\beta_{\mathrm{i}} \lesssim 0.1, h_{\mathrm{pk}}$ increased with increasing $\beta_{\mathrm{i}}$. This is because the deformation of the core is larger for faster rotators. However, for models with larger $\beta_{\mathrm{i}}, h_{\mathrm{pk}}$ decreases as $\beta_{\mathrm{i}}$ increases. These models bounce at subnuclear

Living Reviews in Relativity

http: //www. livingreviews. org/Irr-2003-2 
densities. Thus, the resulting acceleration at bounce and the GW amplitude are smaller. Zwerger and Müller found that the maximum value of $h_{\mathrm{pk}}$ for a given sequence was reached when $\rho_{\mathrm{c}}$ at bounce was just less than $\rho_{\text {nuc }}$. The degree of differential rotation did not have a large effect on the emitted waveforms computed by Zwerger and Müller. However, they did find that models with soft equations of state emitted stronger signals as the degree of differential rotation increased.

The models of Zwerger and Müller that produced the largest GW signals fell into two categories: those with stiff equations of state and $\beta_{\mathrm{i}}<0.01$; and those with soft equations of state, $\beta_{\mathrm{i}} \geq 0.018$, and large degrees of differential rotation. The GW amplitudes emitted during their simulations were in the range $4 \times 10^{-25} \lesssim h \lesssim 4 \times 10^{-23}$, for $d=10 \mathrm{Mpc}$ (the model with the highest $h$ is identified in Figure 2). The corresponding energies ranged from $10^{44} \lesssim E_{\mathrm{GW}} \lesssim 10^{47} \mathrm{erg}$. The peaks of their power spectra were between $500 \mathrm{~Hz}$ and $1 \mathrm{kHz}$. Such signals would fall just outside of the range of LIGO-II. Magnetic fields lower these amplitudes by $\sim 10 \%$ [145], but realistic stellar profiles can lower the amplitudes by a factor of $\gtrsim 4-10$ [190,177], restricting the detectability of supernovae to within our Galaxy ( $\lesssim 10 \mathrm{kpc})$.

Yamada and Sato [264] used techniques very similar to those of Zwerger and Müller [271] in their core collapse study. Their investigation revealed that the $h_{\mathrm{pk}}$ for Type I signals became saturated when the dimensionless angular momentum of the collapsing core, $q=J /(2 G M / c)$, reached $\sim 0.5$. They also found that $h_{\mathrm{pk}}$ was sensitive to the stiffness of the equation of state for densities just below $\rho_{\text {nuc }}$. The characteristics of the GW emission from their models were similar to those of Zwerger and Müller.

\subsubsection{Non-axisymmetric simulations}

The GW emission from non-axisymmetric hydrodynamics simulations of stellar collapse was first studied by Bonazzola and Marck [163, 28]. They used a Newtonian, pseudo-spectral hydrodynamics code to follow the collapse of polytropic models. Their simulations covered only the pre-bounce phase of the collapse. They found that the magnitudes of $h_{\mathrm{pk}}$ in their 3D simulations were within a factor of two of those from equivalent 2D simulations and that the gravitational radiation efficiency did not depend on the equation of state.

The first 3D hydrodynamics collapse simulations to study the GW emission well beyond the core bounce phase were performed by Rampp, Müller, and Ruffert [198]. These authors started their Newtonian simulations with the only model (A4B5G5) of Zwerger and Müller [271] that had a post-bounce value for the stability parameter $\beta=0.35$ that significantly exceeded 0.27 (recall this is the value at which the dynamical bar instability sets in for MacLaurin spheroid-like models). This model had the softest EOS $\left(\Gamma_{\mathrm{r}}=1.28\right)$, highest $\beta_{\mathrm{i}}=0.04$, and largest degree of differential rotation of all of Zwerger and Müller's models. The model's initial density distribution had an off-center density maximum (and therefore a torus-like structure). Rampp, Müller, and Ruffert evolved this model with a $2 \mathrm{D}$ hydrodynamics code until its $\beta$ reached $\approx 0.1$. At that point, $2.5 \mathrm{~ms}$ prior to bounce, the configuration was mapped onto a $3 \mathrm{D}$ nested cubical grid structure and evolved with a 3D hydrodynamics code.

Before the 3D simulations started, non-axisymmetric density perturbations were imposed to seed the growth of any non-axisymmetric modes to which the configuration was unstable. When the imposed perturbation was random (5\% in magnitude), the dominant mode that arose was $m=4$. The growth of this particular mode was instigated by the cubical nature of the computational grid. When an $m=3$ perturbation was imposed (10\% in magnitude), three clumps developed during the post-bounce evolution and produced three spiral arms. These arms carried mass and angular momentum away from the center of the core. The arms eventually merged into a bar-like structure (evidence of the presence of the $m=2$ mode). Significant non-axisymmetric structure was visible only within the inner $40 \mathrm{~km}$ of the core. Their simulations were carried out to $\sim 14 \mathrm{~ms}$ after bounce. 
The amplitudes of the emitted gravitational radiation (computed in the quadrupole approximation) were only $\sim 2 \%$ different from those observed in the 2D simulation of Zwerger and Müller. Because of low angular resolution in the 3D runs, the energy emitted was only $65 \%$ of that emitted in the corresponding $2 \mathrm{D}$ simulation.

The findings of Centrella et al. [49] indicate it is possible that some of the post-bounce configurations of Zwerger and Müller, which have lower values of $\beta$ than the model studied by Rampp, Müller, and Ruffert [198], may also be susceptible to non-axisymmetric instabilities. Centrella et al. have performed 3D hydrodynamics simulations of $\Gamma=1.3$ polytropes to test the stability of configurations with off-center density maxima (as are present in many of the models of Zwerger and Müller [271]). The simulations carried out by Centrella and collaborators were not full collapse simulations, but rather began with differentially rotating equilibrium models. These simulations tracked the growth of any unstable non-axisymmetric modes that arose from the initial $1 \%$ random density perturbations that were imposed. Their results indicate that such models can become dynamically unstable at values of $\beta \gtrsim 0.14$. The observed instability had a dominant $m=1$ mode. Centrella et al. estimate that if a stellar core of mass $M \sim 1.4 M_{\odot}$ and radius $R \sim 200 \mathrm{~km}$ encountered this instability, the values of $h_{\mathrm{pk}}$ from their models would be $\sim 2 \times 10^{-24}-2 \times 10^{-23}$, for $d=10 \mathrm{Mpc}$. The frequency at which $h_{\mathrm{pk}}$ occurred in their simulations was $\sim 200 \mathrm{~Hz}$. This instability would have to persist for at least $\sim 15$ cycles to be detected with LIGO-II.

Brown [35] carried out an investigation of the growth of non-axisymmetric modes in postbounce cores that was similar in many respects to that of Rampp, Müller, and Ruffert [198]. He performed 3D hydrodynamical simulations of the post-bounce configurations resulting from 2D simulations of core collapse. His pre-collapse initial models are $\Gamma=4 / 3$ polytropes in rotational equilibrium. The differential rotation laws used to construct Brown's initial models were motivated by the stellar evolution study of Heger, Langer, and Woosley [109]. The angular velocity profiles of their pre-collapse progenitors were broad and Gaussian-like. Brown's initial models had peak angular velocities ranging from 0.8 - 2.4 times those of [109]. The model evolved by Rampp, Müller, and Ruffert [198] had much stronger differential rotation than any of Brown's models. To induce collapse, Brown reduced the adiabatic index of his models to $\Gamma=1.28$, the same value used by [198].

Brown found that $\beta$ increased by a factor $\lesssim 2$ during his $2 \mathrm{D}$ collapse simulations. This is much less than the factor of $\sim 9$ observed in the model studied by Rampp, Müller, and Ruffert [198]. This is likely a result of the larger degree of differential rotation in the model of Rampp et al.

Brown performed 3D simulations of the two most rapidly rotating of his post-bounce models (models $\Omega 24$ and $\Omega 20$, both of which had $\beta>0.27$ after bounce) and of the model of Rampp et al. (which, although it starts out with $\beta=0.35$, has a sustained $\beta<0.2$ ). Brown refers to the Rampp et al. model as model RMR. Because Brown's models do not have off-center density maxima, they are not expected to be unstable to the $m=1$ mode observed by Centrella et al. [49]. He imposed random $1 \%$ density perturbations at the start of all three of these 3D simulations (note that this perturbation was of a much smaller amplitude than those imposed by [198]).

Brown's simulations determined that both his most rapidly rotating model $\Omega 24$ (with postbounce $\beta>0.35$ ) and model RMR are unstable to growth of the $m=2$ bar-mode. However, his model $\Omega 20$ (with post-bounce $\beta>0.3$ ) was stable. Brown observed no dominant $m=3$ or $m=4$ modes growing in model RMR at the times at which they were seen in the simulations of Rampp et al. This suggests that the mode growth in their simulations was a result of the large perturbations they imposed. The $m=2$ mode begins to grow in model RMR at about the same time as Rampp et al. stopped their evolutions. No substantial $m=1$ growth was observed.

The results of Brown's study indicate that the overall $\beta$ of the post-bounce core may not be a good diagnostic for the onset of instability. He found, as did Rampp, Müller, and Ruffert [198], that only the innermost portion of the core (with $\rho>10^{10} \mathrm{~g} \mathrm{~cm}^{-3}$ ) is susceptible to the bar-mode. This is evident in the stability of his model $\Omega 20$. This model had an overall $\beta>0.3$, but an inner core with $\beta_{\text {ic }}=0.15$. Brown also observed that the $\beta$ of the inner core does not have to exceed 0.27

Living Reviews in Relativity

http://www. livingreviews . org/Irr-2003-2 
for the model to encounter the bar-mode. Models $\Omega 24$ and RMR had $\beta_{\mathrm{ic}} \approx 0.19$. He speculates that the inner cores of these later two models may be bar-unstable because interaction with their outer envelopes feeds the instability or because $\beta_{\mathrm{d}}<0.27$ for such configurations.

Fryer and Warren [91] performed the first 3D collapse simulations to follow the entire collapse through explosion. They used a smoothed particle hydrodynamics code, a realistic equation of state, the flux-limited diffusion approximation for neutrino transport, and Newtonian spherical gravity. Their initial model was nonrotating. Thus, no bar-mode instabilities could develop during their simulations. The only GW emitting mechanism present in their models was convection in the core. The maximum amplitude $h$ of this emission, computed in the quadrupole approximation, was $\sim 3 \times 10^{-26}$, for $d=10 \mathrm{Mpc}$ [88]. In later work, Fryer \& Warren [92] included full Newtonian gravity through a tree algorithm and studied the rotating progenitors from Fryer \& Heger [83]. By the launch of the explosion, no bar instabilities had developed. This was because of several effects: they used slowly rotating, but presumably realistic, progenitors [83], the explosion occured quickly for their models $(\lesssim 100 \mathrm{~ms})$ and, finally, because much of the high angular momentum material did not make it into the inner core. These models have been further studied for the GW signals [87]. The fastest rotating models achieved a signal of $h \sim 2 \times 10^{-24}$ for $d=10 \mathrm{Mpc}$ and characteristic frequencies of $f_{\mathrm{GW}} \sim 1000 \mathrm{~Hz}$. For supernovae occuring within the Galaxy, such a signal is detectable by LIGO-II.

Fryer and collaborators have also modeled asymmetric collapse and asymmetric explosion calculations in 3 dimensions [80, 90]. These calculations will be discussed in Section 3.4.5.

The GW emission from nonradial quasinormal mode oscillations in proto-neutron stars has been examined by Ferrari, Miniutti, and Pons [70]. They found that the frequencies of emission $f_{\mathrm{GW}}$ during the first second after formation $(600-1100 \mathrm{~Hz}$ for the first fundamental and gravity modes) are significantly lower than the corresponding frequencies for cold neutron stars and thus reside in the bandwidths of terrestrial interferometers. However, for first generation interferometers to detect the GW emission from an oscillating proto-neutron star located at $10 \mathrm{Mpc}$, with a signal-tonoise ratio of $5, E_{\mathrm{GW}}$ must be $\sim 10^{-3}-10^{-2} M_{\odot} c^{2}$. It is unlikely that this much energy is stored in these modes (the collapse itself may only emit $\sim 10^{-7} M_{\odot} c^{2}$ in gravitational waves [60]).

\subsubsection{General relativistic simulations}

General relativistic effects oppose the stabilizing influence of rotation in pre-collapse cores. Thus, stars that might be prevented from collapsing due to rotational support in the Newtonian limit may collapse when general relativistic effects are considered. Furthermore, general relativity will cause rotating stars undergoing collapse to bounce at higher densities than in the Newtonian case $[239,271,198,37]$.

The full collapse simulations of Fryer and Heger [83] are the most sophisticated axisymmetric simulations from which the resultant GW emission has been studied [86, 88]. Fryer and Heger include the effects of general relativity, but assume (for the purposes of their gravity treatment only) that the mass distribution is spherical. The GW emission from these simulations was evaluated with either the quadrupole approximation or simpler estimates (see below).

The work of Fryer and Heger [83] is an improvement over past collapse investigations because it starts with rotating progenitors evolved to collapse with a stellar evolution code (which incorporates angular momentum transport via an approximate diffusion scheme) [107], incorporates realistic equations of state and neutrino transport, and follows the collapse to late times. The values of total angular momentum of the inner cores of Fryer and Heger $\left(0.95-1.9 \times 10^{49} \mathrm{~g} \mathrm{~cm}^{2} \mathrm{~s}^{-1}\right)$ are lower than has often been assumed in studies of the GW emission from core collapse. Note that the total specific angular momentum of these core models may be lower by about a factor of 10 if magnetic fields were included in the evolution of the progenitors [3, 232, 110].

FHH's [86] numerical quadrupole estimate of the GWs from polar oscillations in the collapse

Living Reviews in Relativity

http: //www . livingreviews . org/lrr-2003-2 
simulations of Fryer and Heger [83] predicts a peak dimensionless amplitude $h_{\mathrm{pk}}=4.1 \times 10^{-23}$ (for $d=10 \mathrm{Mpc}$ ), emitted at $f_{\mathrm{GW}} \approx 20 \mathrm{~Hz}$. The radiated energy $E_{\mathrm{GW}} \sim 2 \times 10^{44} \mathrm{erg}$. This signal would be just out of the detectability range of the LIGO-II detector.

The cores in the simulations of Fryer and Heger [83] are not compact enough (or rotating rapidly enough) to develop bar instabilities during the collapse and initial bounce phases. However, the explosion phase ejects a good deal of low angular momentum material along the poles in their evolutions. Therefore, about $1 \mathrm{~s}$ after the collapse, $\beta$ becomes high enough in their models to exceed the secular bar instability limit. The $\beta$ of their model with the least angular momentum actually exceeds the dynamical bar instability limit as well (it contracts to a smaller radius and thus has a higher spin rate than the model with higher angular momentum). FHH (and [88]) compute an upper limit (via Equation (2)) to the emitted amplitude from their dynamically unstable model of $h \sim 3 \times 10^{-22}$ (if coherent emission from a bar located at $10 \mathrm{Mpc}$ persists for 100 cycles). The corresponding frequency and maximum power are $f_{\mathrm{GW}} \approx 10^{3} \mathrm{~Hz}$ and $P_{\mathrm{GW}}=10^{53} \mathrm{erg} \mathrm{s}^{-1}$. LIGO-II should be able to detect such a signal (see Figure 2, where FHH's upper limit to $h$ for this dynamical bar-mode is identified).

As mentioned above, the proto-neutron stars of Fryer and Heger are likely to be unstable to the development of secular bar instabilities. The GW emission from proto-neutron stars that are secularly unstable to the bar-mode has been examined by Lai and Shapiro [148, 146]. Because the timescale for secular evolution is so long, 3D hydrodynamics simulations of the nonlinear development of a secular bar can be impractical. To bypass this difficulty, Lai [146] considers only incompressible fluids, for which there are exact solutions for (Dedekind and Jacobi-like) bar development. He predicts that such a bar located at $10 \mathrm{Mpc}$ would emit GWs with a peak characteristic amplitude $h \sim 10^{-21}$, if the bar persists for $10^{2}-10^{4}$ cycles. The maximum $f_{\mathrm{GW}}$ of the emitted radiation is in the range $10^{2}-10^{3} \mathrm{~Hz}$. This type of signal should be easily detected by LIGO-I (although detection may require a technique like the fast chirp transform method of Jenet and Prince [134] due to the complicated phase evolution of the emission).

Alternatively, Ou et al. [191] bypassed the long secular timescale by increasing the driving force of the instability. They found that a bar instability was maintained for several orbits before sheer flows, producing GW emission that would have a signal-to-noise ratio greater than 8 for LIGO-II out to $32 \mathrm{Mpc}$. A movie of this simulation is shown in Figure 6.

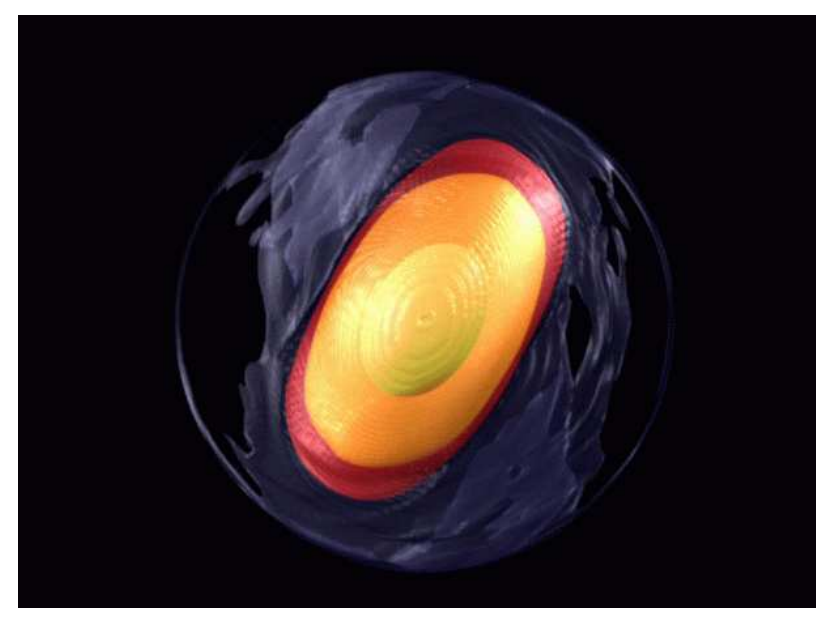

Figure 6: Still from a Movie showing the evolution of a secular bar instability, see Ou et al. [191] for details. (To watch the movie, please go to the online version of this review article at http: //www. livingreviews. org/lrr-2003-2.)

Living Reviews in Relativity

http://www. livingreviews . org/lrr-2003-2 
FHH predict that a fragmentation instability is unlikely to develop during core collapse $\mathrm{SNe}$ because the cores have central density maxima (see also [88]). However, they do give estimates [calculated via Equations $(4,5)$ ] for the amplitude, power, and frequency of the emission from such an instability: $h_{\mathrm{pk}} \sim 2 \times 10^{-22}, P_{\mathrm{GW}}=10^{54} \mathrm{erg} \mathrm{s}^{-1}, f_{\mathrm{GW}} \approx 2 \times 10^{3} \mathrm{~Hz}$. Again, this signal would fall just beyond the upper limit of LIGO-II's frequency range.

The GW emission from $r$-mode unstable neutron star remnants of core collapse $\mathrm{SNe}$ would be easily detectable if $\alpha_{\max } \sim 1$ (which is likely not physical; see Section 2.3). Multiple GW bursts will occur as material falls back onto the neutron star and results in repeat episodes of $r$-mode growth (note that a single $r$-mode episode can have multiple amplitude peaks [153]). FHH calculate that the characteristic amplitude of the GW emission from this $r$-mode evolution tracks from $6-1 \times 10^{-22}$, over a frequency range of $10^{3}-10^{2} \mathrm{~Hz}$ (see Section 2.4 for details). They estimate the emitted energy to exceed $10^{52} \mathrm{erg}$.

General relativity has been more fully accounted for in the core collapse studies of Dimmelmeier, Font, and Müller [58, 59, 60] and Shibata and collaborators [230], which build on the Newtonian, axisymmetric collapse simulations of Zwerger and Müller [271]. In all, they have followed the collapse evolution of 26 different models, with both Newtonian and general relativistic simulations. As in the work of Zwerger and Müller, the different models are characterized by varying degrees of differential rotation, initial rotation rates, and adiabatic indices. They use the conformally flat metric to approximate the space time geometry [56] in their relativistic hydrodynamics simulations. This approximation gives the exact solution to Einstein's equations in the case of spherical symmetry. Thus, as long as the collapse is not significantly aspherical, the approximation is relatively accurate. However, the conformally flat condition does eliminate GW emission from the spacetime. Because of this, Dimmelmeier, Font, and Müller used the quadrupole approximation to compute the characteristics of the emitted GW signal (see [271] for details).

The general relativistic simulations of Dimmelmeier et al. showed the three different types of collapse evolution (and corresponding gravitational radiation signal) seen in the Newtonian simulations of Zwerger and Müller (regular collapse - Type I signal; multiple bounce collapse Type II signal; and rapid collapse - Type III signal). However, relativistic effects sometimes led to a different collapse type than in the Newtonian case. This is because general relativity did indeed counteract the stabilizing effects of rotation and led to much higher bounce densities (up to $700 \%$ higher). They found that multiple bounce collapse is much rarer in general relativistic simulations (occurring in only two of their models). When multiple bounce does occur, relativistic effects shorten the time interval between bounces by up to a factor of four. Movies of the simulations of four models from Dimmelmeier et al. [60] are shown in Figures 7, 8, 9, and 10. The four evolutions shown include a regular collapse (Movie 7), a rapid collapse (Movie 8), a multiple bounce collapse (Movie 9), and a very rapidly and differentially rotating collapse (Movie 10). The left frames of each movie contain the 2D evolution of the logarithmic density. The upper and lower right frames display the evolutions of the gravitational wave amplitude and the maximum density, respectively. These movies can also be viewed at [165].

Dimmelmeier et al. found that models for which the collapse type was the same in both Newtonian and relativistic simulations had lower GW amplitudes $h_{\mathrm{pk}}$ in the relativistic case. This is because the Newtonian models were less compact at bounce and thus had material with higher densities and velocities at larger radii. Both higher and lower values of $h_{\mathrm{pk}}$ were observed in models for which the collapse type changed. Overall, the range of $h_{\mathrm{pk}}\left(4 \times 10^{-24}-3 \times 10^{-23}\right.$, for a source located at $10 \mathrm{Mpc}$ ) seen in the relativistic simulations was quite close to the corresponding Newtonian range. The average $E_{\mathrm{GW}}$ was somewhat higher in the relativistic case $\left(1.5 \times 10^{47} \mathrm{erg}\right.$ compared to the Newtonian value of $6.4 \times 10^{46} \mathrm{erg}$ ). The overall range of GW frequencies observed in their relativistic simulations $(60-1000 \mathrm{~Hz})$ was close to the Newtonian range. They did note that relativistic effects always caused the characteristic frequency of emission, $f_{\mathrm{GW}}$, to increase (up to five-fold). Studies of non-linear pulsations in neutron stars expect high frequencies between 

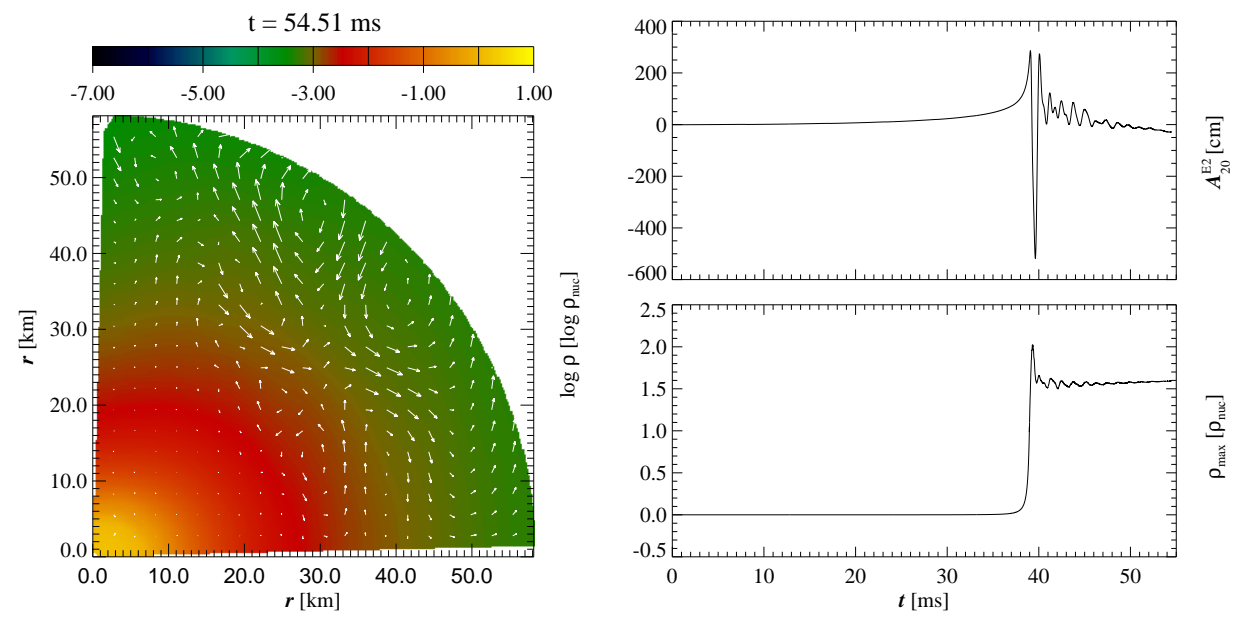

Figure 7: Still from a Movie showing the evolution of the regular collapse model A3B2G4 of Dimmelmeier et al. [60]. The left frame contains the $2 D$ evolution of the logarithmic density. The upper and lower right frames display the evolutions of the gravitational wave amplitude and the maximum density, respectively. (To watch the movie, please go to the online version of this review article at http: // www. livingreviews. org/ Irr-2003-2.)
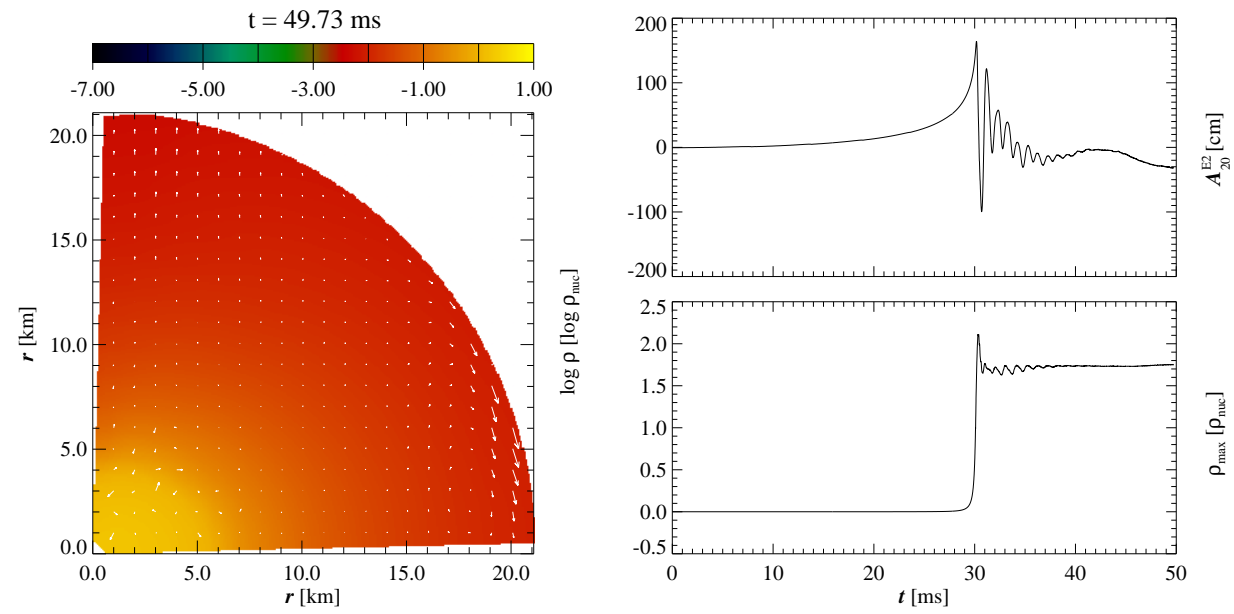

Figure 8: Still from a Movie showing the same as Movie 7, but for rapid collapse model A3B2G5 of Dimmelmeier et al. [60]. (To watch the movie, please go to the online version of this review article at http: //www. livingreviews. org/lrr-2003-2.)

Living Reviews in Relativity

http://www. livingreviews. org/lrr-2003-2 

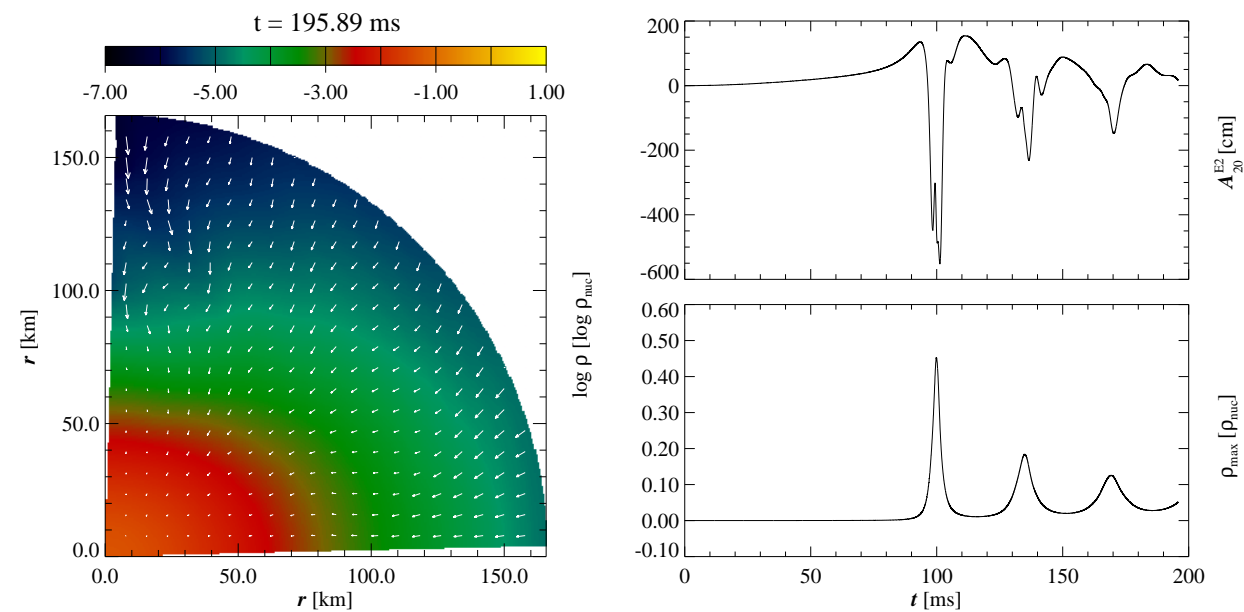

Figure 9: Still from a Movie showing the same as Movie 7, but for multiple collapse model A2B4G1 of Dimmelmeier et al. [60]. (To watch the movie, please go to the online version of this review article at http: //www. livingreviews. org/lrr-2003-2.)
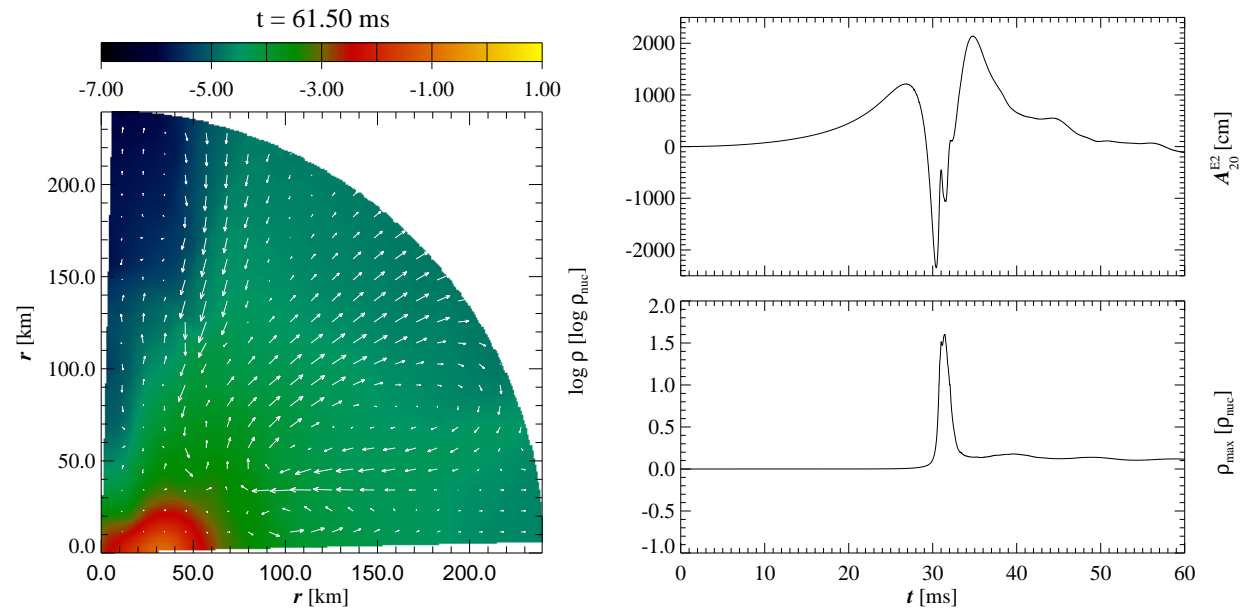

Figure 10: Still from a Movie showing the same as Movie 7, but for rapid, differentially rotating collapse model A4B5G5 of Dimmelmeier et al. [60]. (To watch the movie, please go to the online version of this review article at http: //www. livingreviews. org/ Irr-2003-2.) 
$1.8-3.6 \mathrm{kHz}$ [235]. For most of their models, this increase in $f_{\mathrm{GW}}$ was not accompanied by an increase in $h_{\mathrm{pk}}$. This means that relativistic effects could decrease the detectability of GW signals from some core collapses. However, the GW emission from the models of Dimmelmeier et al. could be detected by the first generation of ground-based interferometric detectors if the sources were fortuitously located in the Local Group of galaxies. A catalog containing the signals and spectra of the GW emission from all of their models can be found at [164].

\subsubsection{Simulations of convective instabilities}

Convectively driven inhomogeneities in the density distribution of the outer regions of the nascent neutron star and anisotropic neutrino emission are other sources of GW emission during the collapse/explosion [41, 173]. GW emission from these processes results from small-scale asphericities, unlike the large-scale motions responsible for GW emission from aspherical collapse and non-axisymmetric global instabilities. Note that Rayleigh-Taylor instabilities also induce timedependent quadrupole moments at composition interfaces in the stellar envelope. However, the resultant GW emission is too weak to be detected because the Rayleigh-Taylor instabilities occur at very large radii [173].

Since convection was suggested as a key ingredient into the explosion, it has been postulated that asymmetries in the convection can produce the large proper motions observed in the pulsar population [111]. Convection asymmetries can either be produced by asymmetries in the progenitor star that grow during collapse or by instabilities in the convection itself. Burrows and Hayes [41] proposed that asymmetries in the collapse could produce the pulsar velocities. The idea behind this work was that asymmetries present in the star prior to collapse (in part due to convection during silicon and oxygen burning) will be amplified during the collapse [20,147]. These asymmetries will then drive asymmetries in the convection and ultimately, the supernova explosion. Burrows and Hayes [41] found that not only could they produce strong motions in the nascent neutron star, but detectable gravitational wave signals. The peak amplitude calculated was $h_{\mathrm{pk}} \sim 3 \times 10^{-24}$, for a source located at $10 \mathrm{Mpc}$.

Fryer [80] was unable to produce the large neutron star velocities seen by Burrows and Hayes [41] even after significantly increasing the level of asymmetry in the initial star in excess of $25 \%$. This discrepancy is now known to be due to the crude 2-dimensional model and gravity scheme used by Burrows and Hayes [40]. However, the gravitational wave signal produced by both simulations is comparable. Figure 11 shows the gravitational waveform from the Burrows \& Hayes simulation (including separate matter and neutrino contributions). Figures 12, 13 show the matter and neutrino contributions respectively to the gravitational wave forms for the Fryer results. The gravitational wave amplitude is dominated by the neutrino component and can exceed $h_{\mathrm{pk}} \sim$ $6 \times 10^{-24}$, for a source located at $10 \mathrm{Mpc}$ in Fryer's most extreme example.

The study by Nazin and Postnov [183] predicts a lower limit for $E_{\mathrm{GW}}$ emitted during an asymmetric core collapse SN (where such asymmetries could be induced by both aspherical mass motion and neutrino emission). They assume that observed pulsar kicks are solely due to asymmetric collapse. They suggest that the energy associated with the kick $\left(M v^{2} / 2\right.$, where $M$ and $v$ are the mass and velocity of the neutron star) can be set as a lower limit for $E_{\mathrm{GW}}$ (which can be computed without having to know the mechanism behind the asymmetric collapse). From observed pulsar proper motions, they estimate the degree of asymmetry $\epsilon$ present in the collapse and the corresponding characteristic GW amplitude $(h \propto \sqrt{\epsilon})$. This amplitude is $3 \times 10^{-25}$ for a source located at $10 \mathrm{Mpc}$ and emitting at $f_{\mathrm{GW}}=1 \mathrm{kHz}$.

Müller and Janka performed both 2D and 3D simulations of convective instabilities in the proto-neutron star and hot bubble regions during the first second of the explosion phase of a Type II SN [176]. They numerically computed the GW emission from the convection-induced aspherical

Living Reviews in Relativity

http: //www. livingreviews.org/lrr-2003-2 


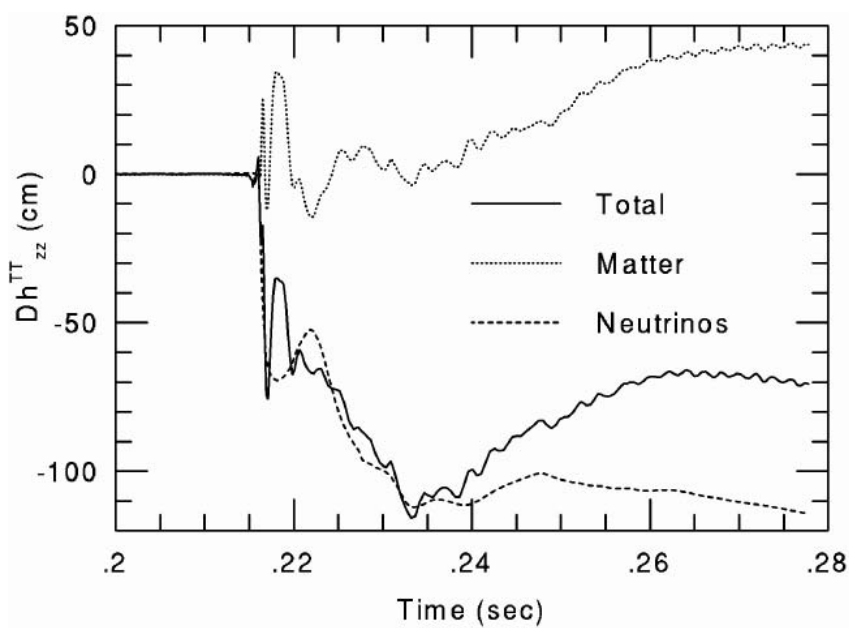

Figure 11: The gravitational waveform (including separate matter and neutrino contributions) from the collapse simulations of Burrows and Hayes [41]. The curves plot the gravitational wave amplitude of the source as a function of time. (Figure 3 of [41]; used with permission.)
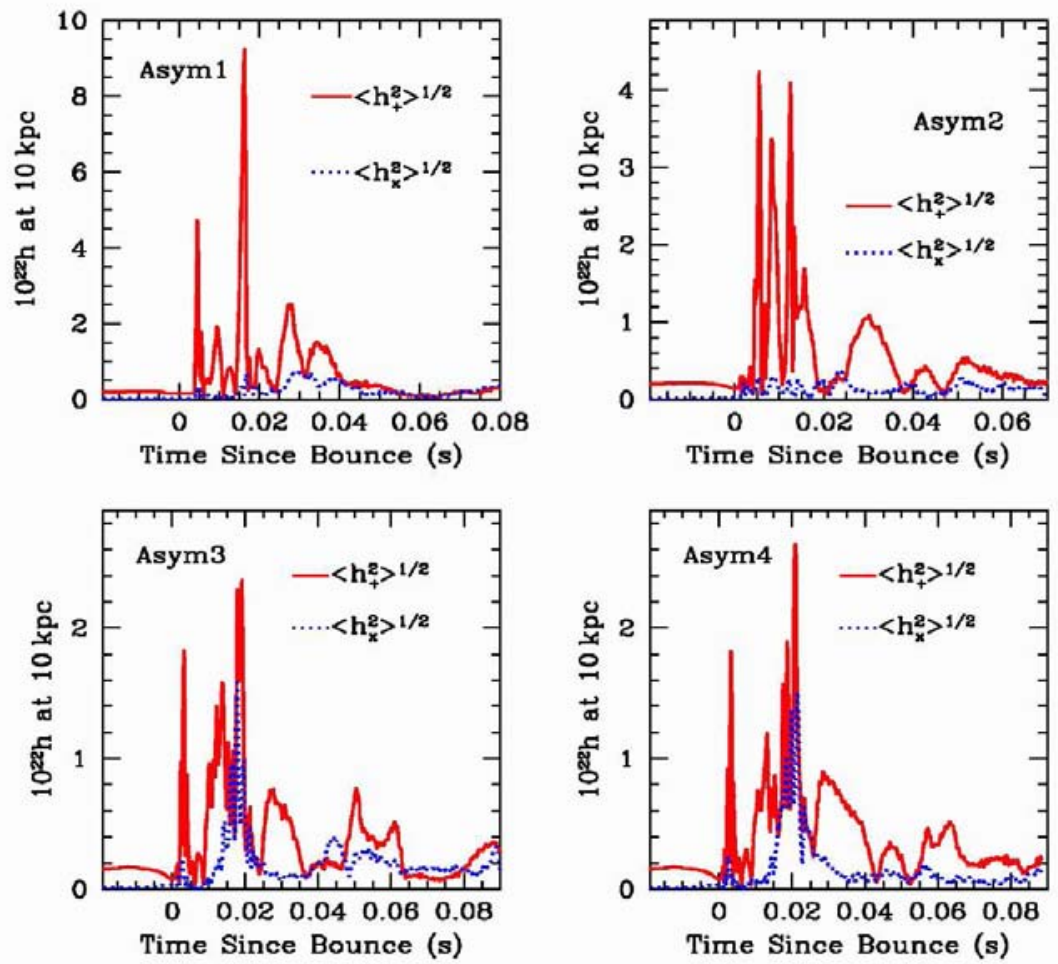

Figure 12: The gravitational waveform for matter contributions from the asymmetric collapse simulations of Fryer et al. [87]. The curves plot the the gravitational wave amplitude of the source as a function of time. (Figure 3 of [87]; used with permission.) 


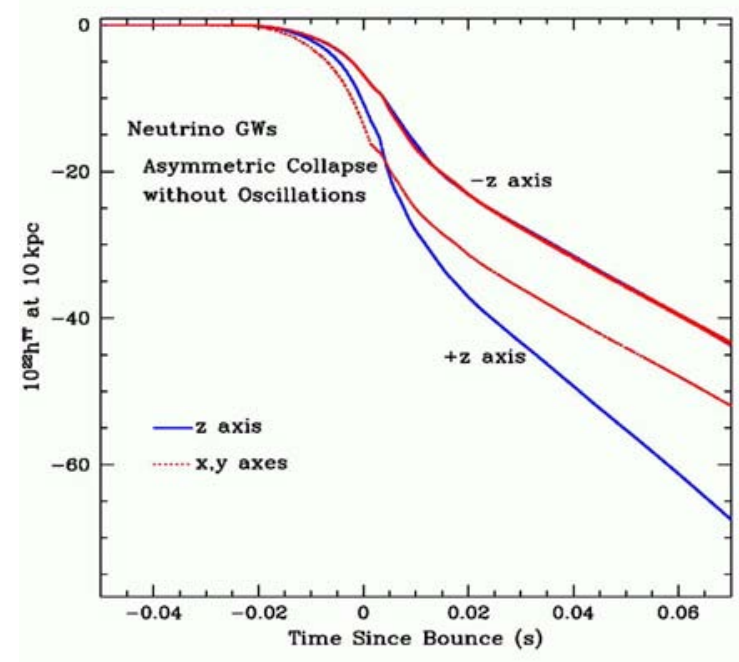

Figure 13: The gravitational waveform for neutrino contributions from the asymmetric collapse simulations of Fryer et al. [87]. The curves plot the product of the gravitational wave amplitude to the source as a function of time. (Figure 8 of [87]; used with permission.)

mass motion and neutrino emission in the quadrupole approximation (for details, see Section 3 of their paper).

For typical iron core masses, the convectively unstable region in the proto-neutron star extends over the inner $0.7-1.20 M_{\odot}$ of the core mass (this corresponds to a radial range of $\sim 10-50 \mathrm{~km}$ ). The convection in this region, which begins approximately 10-20 ms after the shock forms and may last for $\approx 20 \mathrm{~ms}-1 \mathrm{~s}$, is caused by unstable gradients in entropy and/or lepton number resulting from the stalling of the prompt shock and deleptonization outside the neutrino sphere. Müller and Janka's simulations of convection in this region began with the 1D, non-rotating, 12 ms postbounce model of Hillebrandt [113]. This model included general relativistic corrections that had to be relaxed away prior to the start of the Newtonian simulations. Neutrino transport was neglected in these runs (see Section 2.1 of [176] for justification); however, a sophisticated equation of state was utilized. Figure 14 shows the evolution of the temperature and density distributions in the 2D simulation of Müller and Janka.

The peak GW amplitude resulting from convective mass motions in these simulations of the proto-neutron star was $\approx 3 \times 10^{-24}$ in $2 \mathrm{D}$ and $\approx 2 \times 10^{-25}$ in $3 \mathrm{D}$, for $d=10 \mathrm{Mpc}$. More recent calculations get amplitudes of $\approx 10^{-26}$ in $2 \mathrm{D}$ [177] and $\approx 3-5 \times 10^{-26}$ in 3D [87]. The emitted energy was $9.8 \times 10^{44} \mathrm{erg}$ in $2 \mathrm{D}$ and $1.3 \times 10^{42} \mathrm{erg}$ in $3 \mathrm{D}$. The power spectrum peaked at frequencies of $200-600 \mathrm{~Hz}$ in 2D and $100-200 \mathrm{~Hz}$ in 3D. Such signals would not be detectable with LIGO-II. The reasons for the differences between the 2D and 3D results include smaller convective elements and less under- and overshooting in 3D. The relatively low angular resolution of the 3D simulations may have also played a role. The quadrupole gravitational wave amplitude $A_{20}^{\mathrm{E} 2}$ from the $2 \mathrm{D}$ simulation is shown in the upper left panel of Figure 15 (see [271, 241] for expressions relating $A_{20}^{\mathrm{E} 2}$ to $h$ ).

Convection in the hot bubble region between the shock and neutrino sphere arises because of an unstable entropy gradient resulting from the outward moving shock and subsequent neutrino heating. Figure 16 shows a movie of the development of this entropy-driven convection. This unstable region extends over the inner mass range $1.25-1.40 M_{\odot}$ (corresponding to a radial range of $\approx 100-1000 \mathrm{~km})$. Convection in the hot bubble begins $\approx 50-80 \mathrm{~ms}$ after shock formation

Living Reviews in Relativity

http://www. livingreviews.org/lrr-2003-2 


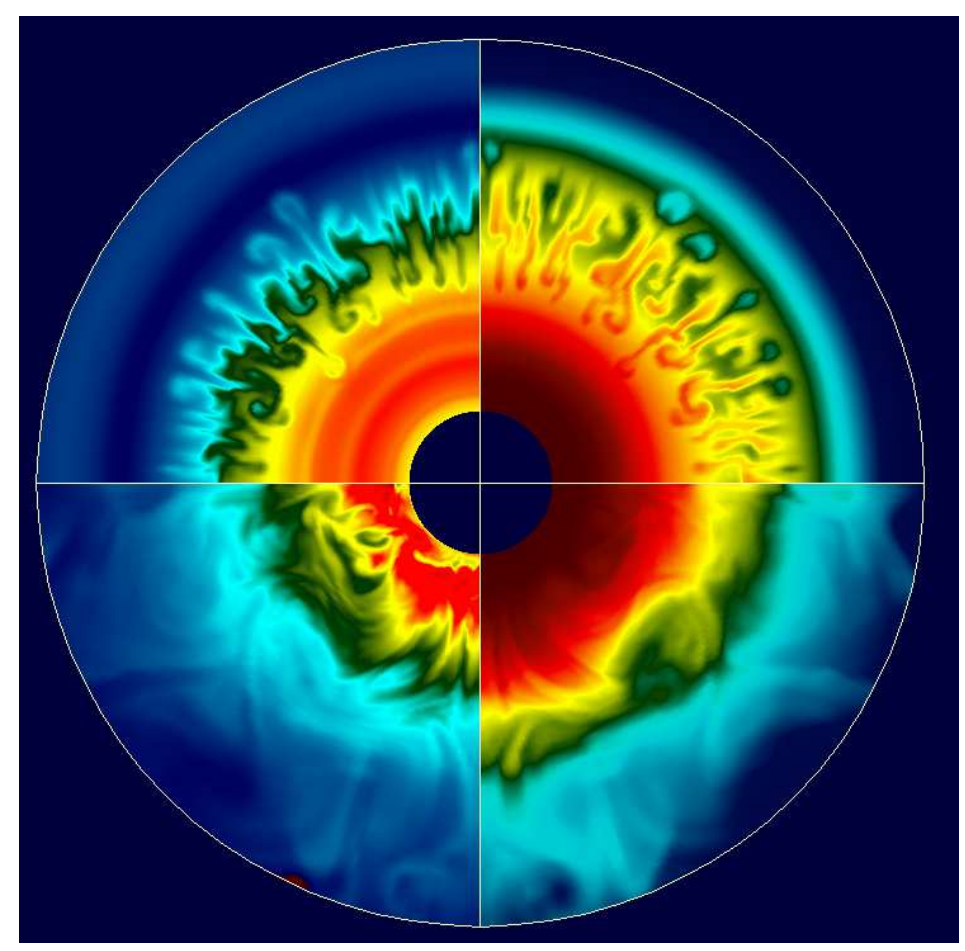

Figure 14: Convective instabilities inside the proto-neutron star in the 2D simulation of Müller and Janka [176]. The evolutions of the temperature (left panels) and logarithmic density (right panels) distributions are shown for the radial region $15-95 \mathrm{~km}$. The upper and lower panels correspond to times 12 and $21 \mathrm{~ms}$, respectively, after the start of the simulation. The temperature values range from $2.5 \times 10^{10}$ to $1.8 \times 10^{11} \mathrm{~K}$. The values of the logarithm of the density range from 10.5 to $13.3 \mathrm{~g} \mathrm{~cm}^{-3}$. The temperature and density both increase as the colors change from blue to green, yellow, and red. (Figure 7 of [176]; used with permission.) 

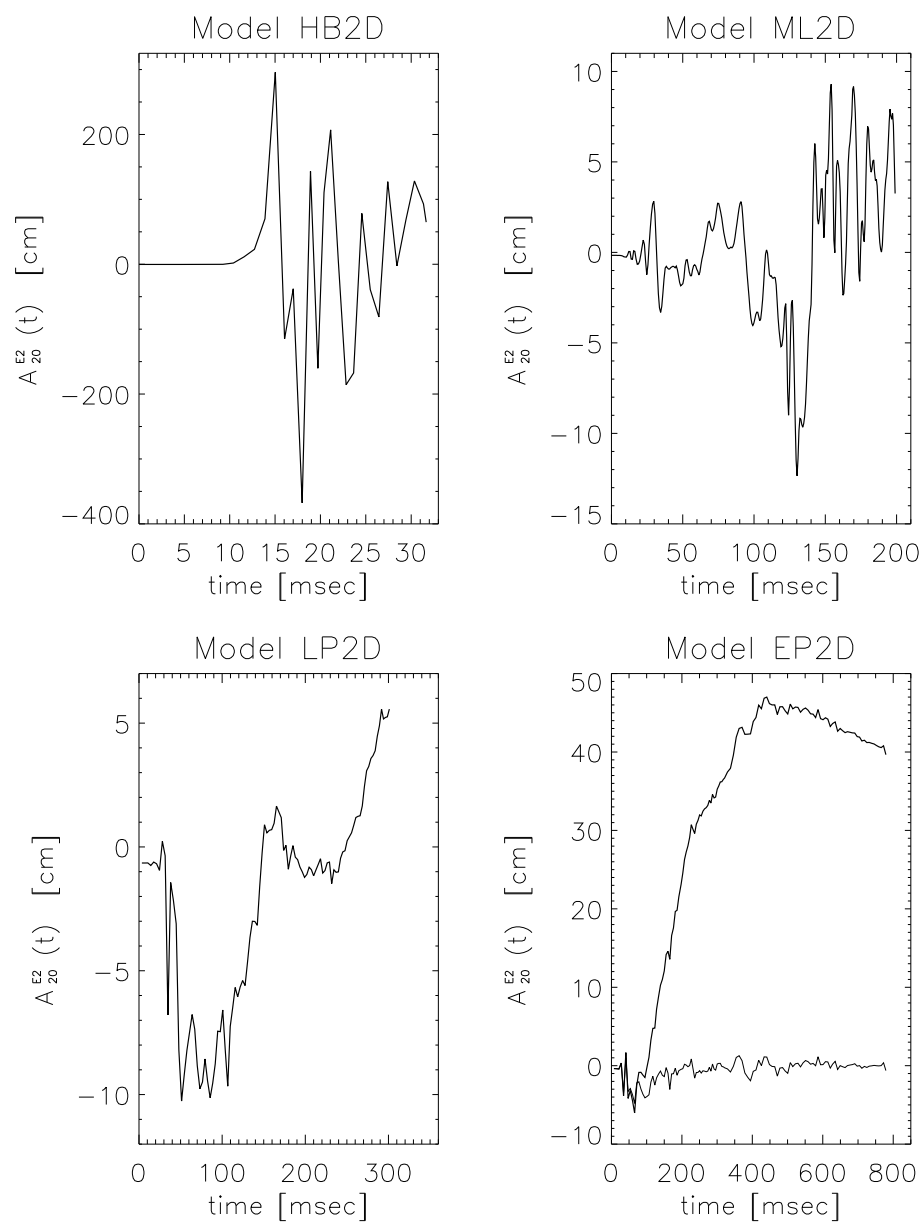

Figure 15: Quadrupole amplitudes $A_{20}^{\mathrm{E} 2}[\mathrm{~cm}]$ from convective instabilities in various models of [176]. The upper left panel is the amplitude from a $2 D$ simulation of proto-neutron star convection. The other three panels are amplitudes from $2 D$ simulations of hot bubble convection. The imposed neutrino flux in the hot bubble simulations increases from the top right model through the bottom right model. (Figure 18 of [176]; used with permission.)

Living Reviews in Relativity

http: //www . livingreviews . org/lrr-2003-2 
and lasts for $\approx 100-500 \mathrm{~ms}$. Only $2 \mathrm{D}$ simulations were performed in this case. These runs started with a 25 ms post-bounce model provided by Bruenn. A simple neutrino transport scheme was used in the runs and an imposed neutrino flux was located inside the neutrino sphere. Due to computational constraints, the computational domain did not include the entire convectively unstable region inside the proto-neutron star (thus this set of simulations only accurately models the convection in the hot bubble region, not in the proto-neutron star).

Figure 16: Still from a Movie showing the isosurface of material with radial velocities of $1000 \mathrm{~km} \mathrm{~s}^{-1}$ for 3 different simulation resolutions. The isosurface outlines the outward moving convective bubbles. The open spaces mark the downflows. Note that the upwelling bubbles are large and have very similar size scales to the two-dimensional simulations. (To watch the movie, please go to the online version of this review article at http: // www. livingreviews. org/ lrr-2003-2.)

The peak GW amplitude resulting from these $2 \mathrm{D}$ simulations of convective mass motions in the hot bubble region was $h_{\mathrm{pk}} \approx 5 \times 10^{-25}$, for $d=10 \mathrm{Mpc}$. The emitted energy was $\lesssim 2 \times 10^{42} \mathrm{erg}$. The energy spectrum peaked at frequencies of $50-200 \mathrm{~Hz}$. As the explosion energy was increased (by increasing the imposed neutrino flux), the violent convective motions turn into simple rapid expansion. The resultant frequencies drop to $f_{\mathrm{GW}} \sim 10 \mathrm{~Hz}$. The amplitude of such a signal would be too low to be detectable with LIGO-II.

The case for GWs from convection induced asymmetric neutrino emission has also varied with time. Müller and Janka estimated the GW emission from the convection induced anisotropic neutrino radiation in their simulations (see [176] for details). They found that the amplitude of the GWs emitted can be a factor of $5-10$ higher than the GW amplitudes resulting from convective 
mass motion. Müller et al. (2004) [177] argue now that the GWs produced by asymmetric neutrino emission is less than that of the convective motions.

Our understanding of the convective engine is evolving with time. Scheck et al. [212] found that the convective cells could merge with time, producing a single lobe convective instability, fulfilling the prediction by Herant [111]. Blondin et al. [26] argue that a standing shock instability could develop to drive low-mode convection and Burrows et al. [43] argue that it is the shocks produced in this convection that truly drives the supernova explosion. This convection can drive oscillations in the neutron star which may also be a source for GWs (see Fig. 17).

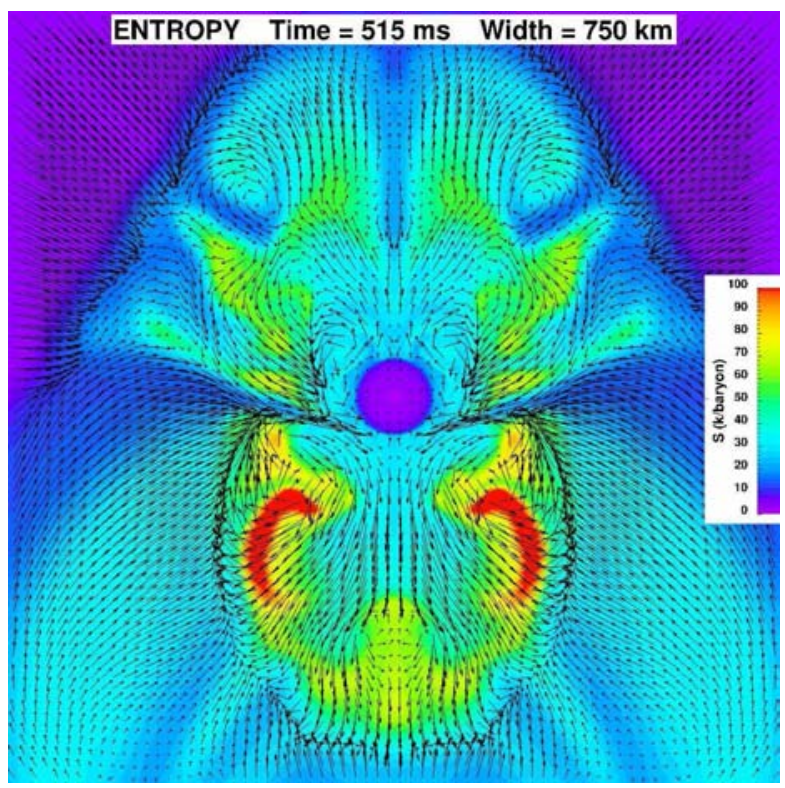

Figure 17: Still from a Movie showing the oscillation of the proto-neutron star caused by acoustic instabilities in the convective region above the shock. (To watch the movie, please go to the online version of this review article at http: // www. livingreviews. org/ $\mathrm{rr}-2003-2$.)

\subsection{Going further}

The background of GW emission from a population of core collapse SNe at cosmological distances may be detectable by LIGO-II, according to Ferrari, Matarrese, and Schneider [69]. They determined the SN rate as a function of redshift using observations to determine the evolution of the star formation rate. Only collapses that lead to black hole formation were considered. This simplified the study because the GW emission from such collapses is generally a function of just the black hole mass and angular momentum. They found that the stochastic background from these sources is not continuous and suggest that this could be used to optimize detection strategies. The maximum GW spectral strain amplitude they computed was in the range $10^{-28}-10^{-27} \mathrm{~Hz}$, at frequencies of a few times $10^{2} \mathrm{~Hz}$. Such a signal may be detected by a pair of LIGO-II detectors. Buonanno et al. [38] have recently redone such a study with all the current results on supernovae, arguing that the GW background would be detectable by second-generation (e.g., Big Bang Observatory) space-based detectors, noting that Pop III (black-hole forming stars) could well dominate the total background.

Living Reviews in Relativity

http: //www. livingreviews. org/Irr-2003-2 


\section{Collapsar Models}

\subsection{Collapse scenario}

Without winds, stars above $\sim 40-50 M_{\odot}$ will not produce supernova explosions, but collapse directly to black holes [78]. Stars above $\sim 20-25 M_{\odot}$ are believed to produce only weak supernova explosions. These explosions are unable to eject all of the star's mantle, and will ultimately also collapse to black holes as this mantle falls back onto the core [78]. If these stars are rotating, they will develop accretion disks around the newly formed black hole, opening up new ways to produce both GWs and baryonic explosions.

These black hole accretion disks formed by stellar collapse are currently the favored engine behind long-duration gamma-ray bursts $[262,160]$. It is believed that either neutrinos rising from the disk annihilate and drive an explosion or magnetic fields wound up in the disk can produce jets that then evolve into the highly relativistic outflows observed in the GRBs [195]. Relativistic and magnetic field effects are far more important in these calculations than those for normal stellar collapse models. And yet, most simulations of GRBs to date do not include the detailed microphysics, magnetic fields (although see Proga and collaborators [196]), and relativity at the same level of sophistication as the simulations of core-collapse supernovae. Even so, this topic has garnered some interest in the GW community and this interest will only grow as the simulations become more accurate.

\subsection{Formation rate}

The fraction of massive stars collapsing to black holes can be estimated theoretically and lies between $5-40 \%$ [89]. But it is much more difficult to determine the fraction of these black-hole forming stars that also have enough rotation to produce a GRB. Likewise, the wide-field monitoring of the night sky of GRBs produces an accurate observed rate of GRBs, but this rate must be corrected by beaming factors to get a true long-duration GRB rate. Observations of supernova remnants have allowed GRB observers to place some constraints on the GRB rate relative to the Type $\mathrm{Ib} / \mathrm{c}$ supernova rate: Rate $_{\mathrm{GRB}} / \mathrm{Rate}_{\mathrm{SNIb} / \mathrm{c}}<3 \%$ [22]. This predicts a Galactic rate that is below $\sim 10^{-4} \mathrm{yr}^{-1}$ and the true value is likely to lie between $\sim 10^{-5}-10^{-4} \mathrm{yr}^{-1}$. Correlating the observed GRB rate to the rate of black hole accretion disks formed in stellar collapse is not straightforward. First, it may be that long-duration GRBs are not formed from stellar collapse, but from stellar mergers [93, 94]. If this is the case, then we do not expect any GW signal from the collapse itself, but any disk instabilities may still produce GW signals. Second, there may be a number of black hole accretion disk systems produced that do not produce gamma-ray bursts because they can not achieve the high relativistic flows, increasing the total rate of GW sources.

\subsection{GW emission mechanisms}

If the collapsed remnant is a black hole, GWs will be radiated as the infall of the remaining stellar matter distorts the black hole's geometry. This "ringdown phase" will end when gravitational radiation has dissipated all of the black hole's accretion-induced distortion. Zanotti, Rezzolla, and Font [269] have suggested that the torus of matter surrounding the black hole may be an even stronger source of GWs than the collapse itself (see also [140, 141, 251, 250]). Kobayashi \& Mészáros (2003) have further found that the GW signal will be polarized and this polarization may be detectable. 


\subsection{Numerical Predictions of GW emission Mechanisms}

The first fully general relativistic investigations of stellar core collapse were Nakamura's 2D simulations of rotating collapse $[179,180]$. However, because of the limits of his numerical formalism and computational resources, he was unable to compute the emitted gravitational radiation (the energy of this emission is quite small compared to the rest mass energy and thus was difficult to extract numerically). The results of this work indicate that collapse does not lead to black hole formation if the parameter $q=J / M^{2}$ exceeds unity (here $J$ and $M$ are the angular momentum and gravitational mass of the remnant).

Fully general relativistic collapse simulations (i.e., without the conformally flat approximation) have also been performed by Shibata [222]. He used an axisymmetric code that solves the Einstein equations in Cartesian coordinates and the hydrodynamics equations in cylindrical coordinates. The use of the Cartesian grid eliminates the presence of singularities and allows for stable, longduration axisymmetric simulations [4]. The focus of this work was the effect of rotation on the criteria for prompt black hole formation. Shibata found that if the parameter $q=J / M^{2}$ is less than 0.5 , black hole formation occurred for rest masses slightly greater than the maximum mass of spherical stars. However, for $0.5<q<1$, the maximum stable rest mass is increased by $\sim 70-80 \%$. The results are only weakly dependent on the initial rotation profile. More recent results suggest that this limit can be eased for differentially rotating massive stellar cores, and systems with spin parameter $q$ as high as 2.5 may collapse to form black holes [217]. Shibata did not compute the GW emission in his collapse simulations, but in a recent study using axisymmetric calculations, GW signals have been calculataed focusing on this collapse phase [267].

Duez et al. [62] found that if a black hole does form, but the disk is spinning rapidly, that the disk will fragment and its subsequent accretion will be in spurts, causing a "splash" onto the black hole, producing ringing and GW emission. Their result implies very strong gravitational wave amplitudes $\gtrsim 10^{-21}$ at distances of $10 \mathrm{Mpc}$. Black hole ringing was also estimated by FHH, where they too assumed discrete accretion events. They found that even with very optimistic accretion scenarios, that such radiation will be of very low amplitude and beyond the upper frequency reach of LIGO-II (see [86] for details).

The new general relativistic hydrodynamics simulations of Zanotti, Rezzolla, and Font [269] suggest that a torus of neutron star matter surrounding a black hole remnant may be a stronger source of GWs than the collapse itself. They used a high resolution shock-capturing hydrodynamics method in conjunction with a static (Schwarzschild) spacetime to follow the evolution of "toroidal neutron stars". Their results indicate that if a toroidal neutron star (with constant specific angular momentum) is perturbed, it could undergo regular oscillations. They estimate that the resulting GW emission would have a characteristic amplitude $h_{\mathrm{c}}$ ranging from $6 \times 10^{-24}-5 \times 10^{-23}$, for ratios of torus mass to black hole mass in the range $0.1-0.5$. (These amplitude values are likely underestimated because the simulations of Zanotti et al. are axisymmetric.) The corresponding frequency of emission is $f_{\mathrm{GW}} \sim 200 \mathrm{~Hz}$. The values of $h_{\mathrm{c}}$ and $f_{\mathrm{GW}}$ quoted here are for a source located at $10 \mathrm{Mpc}$. This emission would be just outside the range of LIGO-II (see Figure 2). Further numerical investigations, which study tori with non-constant angular momenta and include the effects of self-gravity and black hole rotation, are needed to confirm these predictions. Movies from the simulations of Zanotti et al. can be viewed at [200].

Magnetized tori around rapidly spinning black holes (formed via either core collapse or neutron star-black hole coalescence) have recently been examined in the theoretical study of van Putten and Levinson [251]. They find that such a torus-black hole system can exist in a suspended state of accretion if the ratio of poloidal magnetic field energy to kinetic energy $E_{B} / E_{\mathrm{k}}$ is less than 0.1. They estimate that $\sim 10 \%$ of the spin energy of the black hole will be converted to gravitational radiation energy through multipole mass moment instabilities that develop in the torus. If a magnetized torus-black hole system located at $10 \mathrm{Mpc}$ is observed for $2 \times 10^{4}$ rotation periods,

Living Reviews in Relativity

http://www. livingreviews . org/Irr-2003-2 
the characteristic amplitude of the GW emission is $\sim 6 \times 10^{-20}$. It is possible that this emission could take place at several frequencies. Observations of x-ray lines from gamma-ray bursts (which are possibly produced by these types of systems) could constrain these frequencies by providing information regarding the angular velocities of the tori: preliminary estimates from observations suggest $f_{\mathrm{GW}} \sim 500 \mathrm{~Hz}$, placing the radiation into a range detectable by LIGO-I [251].

\subsection{Going further}

As we noted in Section 3.5, the background of GW emission is likely dominated by the population of stars that collapse to black holes. We refer the reader back to this section on progress in this arena.

Baumgarte and collaborators have studied the neutrino signal from the collapse of a hot neutron star down to a black hole $[18,19,15]$. This collapse phase has a specific neutrino signal, that when compared with the gravitational wave observations, can tell us a lot about the behavior of matter at nuclear densities. 


\section{Collapse of Population III Stars}

\subsection{Collapse scenario}

The first generation of stars to form in the early universe are known as Population III stars (formed at redshifts $z \gtrsim 5$ ). Theoretical and computational evidence suggests that Population III stars may have had masses $\gtrsim 100 M_{\odot}[2,1,96]$. Since these massive stars contained no metals, it was possible for them to form directly and then evolve with very low stellar winds and thus very little mass loss. If the mass of a nonrotating Population III star is $\gtrsim 260 M_{\odot}$, its fate is to collapse directly to a black hole at the end of its life [96]. If rotational support prevents the star from direct collapse to a black hole, explosive thermonuclear burning will cause the star to undergo a giant hypernova explosion. Prior to black hole formation, the rotating, collapsed core will have a mass of $50-70 M_{\odot}$ and a radius of $1000-2000 \mathrm{~km}$. Note that because these massive stars evolve so quickly (in a few million years [10]), the events associated with their deaths will take place at roughly the redshifts of their births.

\subsection{Formation rate}

The formation rate of Population III stars can be indirectly estimated from the re-ionization fraction of the early universe, which was re-ionized by light from these stars [117, 47]. Using estimates of the ultraviolet light emission from Population III stars, their ionization efficiency, and the re-ionization fraction of the early universe, one can determine that about $0.01 \%-1 \%$ of the universe's baryonic matter was found in these very massive stars. This corresponds to $\sim 10^{4}-10^{7}$ Population III stars in a $10^{11} M_{\odot}$ galaxy and thus a collapse rate that is $\lesssim 10^{-3} \mathrm{yr}^{-1}$. Thus, a reasonable occurrence rate can be found for an observation (luminosity) distance of $\sim 50 \mathrm{Gpc}$ (which corresponds to a redshift of $z=5$, in the cosmology used by [86]). However, uncertainties in the assumptions make this formation rate uncertain by a few orders of magnitude $[1,96,2,86]$. Indeed, the latest results suggest that the initial mass function begins to fall off dramatically above $100 M_{\odot}$ and the number of stars fomred with masses above $25 M_{\odot}$ may be many orders of magnitude below this rate[189]. Instead, Population III stars may be dominated by the collapse of stars in the 20-100 $M_{\odot}$. These stars may also collapse ultimately to black holes. For discussion of these objects, we refer the reader back to Section 4 .

\subsection{GW emission mechanisms}

The GW emission mechanisms related to the collapse of Population III stars are a subset of those discussed in the sections on AIC and SNe/collapsars. These include aspherical collapse, global rotational and fragmentation instabilities that may arise during the collapse/explosion and in the collapse remnant (prior to black hole formation), and the "ring-down" of the remnant black hole.

\subsection{Numerical predictions of GW emission}

The GW emission from the collapse of Population III stars has been investigated by Fryer and collaborators (Fryer, Woosley, and Heger [96], FHH [86], and Fryer, Holz, Hughes, and Warren [88]). The collapse simulations of Fryer, Woosley, and Heger again started with rotating collapse progenitors that had been evolved with a stellar evolution code [107]. The initial models used by the evolution code were in rigid rotation with a surface ratio of centrifugal to gravitational forces of $20 \%$ (this ratio is seen in current observations of O stars).

The results of Fryer, Woosley, and Heger suggest that the collapse remnant (prior to black hole formation) is susceptible to the development of a secular bar-mode instability. However, at $z>5$, the GW emission would be redshifted out of LIGO-II's frequency range. At $z=5, h_{\mathrm{pk}}=8 \times 10^{-23}$,

Living Reviews in Relativity

http: //www . livingreviews . org/lrr-2003-2 
with a corresponding frequency of $10 \mathrm{~Hz}[86,88]$. Even if such a signal persists for a hundred cycles, it probably would be undetectable by LIGO-II. Note that these signal strengths are orders of magnitude lower than the qualitative estimates of signal strength given in Carr, Bond, and Arnett [47].

LIGO-II may be able to detect the GW emission from binary clumps formed via a fragmentation instability. If such a signal is emitted at $z=5$ and persists for 10 cycles, $h$ would be $\sim 10^{-22}$, over a frequency range of $10-100 \mathrm{~Hz}[86,88]$. The likelihood of the development of a fragmentation instability is diminished by the fact that the off-center density maxima present in the simulations of Fryer, Woosley, and Heger are not very pronounced.

The "ring-down" of the black hole remnant will likely be strong because Fryer, Woosley, and Heger observe a high accretion rate after collapse. FHH estimate that for a source located at $z=20$, the GWs would be redshifted out of LIGO-II's bandwidth. However, for a source at $z=5$, $h_{\mathrm{pk}} \sim 6 \times 10^{-23}$ and the frequency range is $20-70 \mathrm{~Hz}$. This signal may be marginally detectable with LIGO-II (see Figure 2). 


\section{Collapse of Supermassive Stars}

\subsection{Collapse scenario}

There is a large body of observational evidence that supermassive black holes (SMBHs, $M \gtrsim$ $10^{6} M_{\odot}$ ) exist in the centers of many, if not most galaxies (see, e.g., the reviews of Rees [199] and Macchetto [159]). The masses of SMBHs in the centers of more than 45 galaxies have been estimated from observations [68] and there are more than 30 galaxies in which the presence of a SMBH has been confirmed [142].

One of the possible formation mechanisms for SMBHs involves the gravitational collapse of supermassive stars (SMSs). The timescale for this formation channel is short enough to account for the presence of SMBHs at redshifts $z>6$ [129]. Supermassive stars may contract directly out of the primordial gas, if radiation and/or magnetic field pressure prevent fragmentation [101, 64, 100, $157,33,1]$. Alternatively, they may build up from fragments of stellar collisions in clusters [211, 21]. Supermassive stars are radiation dominated, isentropic and convective [221, 270, 157]. Thus, they are well represented by an $n=3$ polytrope. If the star's mass exceeds $10^{6} M_{\odot}$, nuclear burning and electron/positron annihilation are not important.

After formation, an SMS will evolve through a phase of quasistationary cooling and contraction. If the SMS is rotating when it forms, conservation of angular momentum requires that it spins up as it contracts. There are two possible evolutionary regimes for a cooling SMS. The path taken by an SMS depends on the strength of its viscosity and magnetic fields and on the nature of its angular momentum distribution.

In the first regime, viscosity or magnetic fields are strong enough to enforce uniform rotation throughout the star as it contracts. Baumgarte and Shapiro [16] have studied the evolution of a uniformly rotating SMS up to the onset of relativistic instability. They demonstrated that a uniformly rotating, cooling SMS will eventually spin up to its mass shedding limit. The mass shedding limit is encountered when matter at the star's equator rotates with the Keplerian velocity. The limit can be represented as $\beta_{\text {shed }}=(T /|W|)_{\text {shed }}$. In this case, $\beta_{\text {shed }}=9 \times 10^{-3}$. The star will then evolve along a mass shedding sequence, losing both mass and angular momentum. It will eventually contract to the onset of relativistic instability [123, 50, 51, 221, 129].

Baumgarte and Shapiro used both a second-order, post-Newtonian approximation and a fully general relativistic numerical code to determine that the onset of relativistic instability occurs at a ratio of $R / M \sim 450$, where $R$ is the star's radius and $G=c=1$ in the remainder of this section. Note that a second-order, post-Newtonian approximation was needed because rotation stabilizes the destabilizing role of nonlinear gravity at the first post-Newtonian level. If the mass of the star exceeds $10^{6} M_{\odot}$, the star will then collapse and possibly form a SMBH. If the star is less massive, nuclear reactions may lead to explosion instead of collapse.

The major result of Baumgarte and Shapiro's work is that the universal values of the following ratios exist for the critical configuration at the onset of relativistic instability: $T /|W|, R / M$, and $J / M^{2}$. These ratios are completely independent of the mass of the star or its prior evolution. Because uniformly rotating SMSs will begin to collapse from a universal configuration, the subsequent collapse and the resulting gravitational waveform will be unique.

In the opposite evolutionary regime, neither viscosity nor magnetic fields are strong enough to enforce uniform rotation throughout the cooling SMS as it contracts. In this case, it has been shown that the angular momentum distribution is conserved on cylinders during contraction [27]. Because viscosity and magnetic fields are weak, there is no means of redistributing angular momentum in the star. So, even if the star starts out rotating uniformly, it cannot remain so.

The star will then rotate differentially as it cools and contracts. In this case, the subsequent evolution depends on the star's initial angular momentum distribution, which is largely unknown. One possible outcome is that the star will spin up to mass-shedding (at a different value of $\beta_{\text {shed }}$

Living Reviews in Relativity

http://www. livingreviews.org/lrr-2003-2 
than a uniformly rotating star) and then follow an evolutionary path that may be similar to that described by Baumgarte and Shapiro [16]. The alternative outcome is that the star will encounter the dynamical bar instability prior to reaching the mass-shedding limit. New and Shapiro $[185,186]$ have demonstrated that a bar-mode phase is likely to be encountered by differentially rotating SMSs with a wide range of initial angular momentum distributions. This mode will transport mass and angular momentum outward and thus may hasten the onset of collapse.

\subsection{Formation rate}

An estimate of the rate of the collapse of SMSs can be derived from the quasar luminosity function. Haehnelt [99] has used the quasar luminosity function to compute the rate of GW bursts from supermassive black holes, assuming that each quasar emits one such burst during its lifetime (and that each quasar is a supermassive black hole). If it is assumed that each of these bursts is due to the formation of a supermassive black hole via the collapse of a SMS, then Haehnelt's rate estimates can be used as estimates of the rate of SMS collapse. This rate is likely an overestimate of the SMS collapse rate because many SMBHs may have been formed via merger. Haehnelt predicts that the integrated event rate through redshift $z=4.5$ ranges from $\sim 10^{-6} \mathrm{yr}^{-1}$ for $M=10^{8} M_{\odot}$ objects to $\sim 1 \mathrm{yr}^{-1}$ for $M=10^{6} M_{\odot}$ objects. Thus, as in the case of Population III stars, a reasonable occurrence rate can be determined for an observation (luminosity) distance of $50 \mathrm{Gpc}$.

\subsection{GW emission mechanisms}

The GW emission mechanisms related to the collapse of SMSs are a subset of those discussed in the sections on AIC, SNe/collapsars, and Population III stellar collapse. These include (i) aspherical collapse, (ii) global rotational and fragmentation instabilities that may arise during the collapse/explosion and in the collapsed remnant (prior to black hole formation), and (iii) the "ring-down" of the remnant black hole.

\subsection{Numerical predictions of GW emission}

The outcome of SMS collapse can be determined only with numerical, relativistic 3D hydrodynamics simulations.

Until recently, such simulations had been published only for nearly spherical collapse. The spherical simulations of Shapiro and Teukolsky [220] produced collapse evolutions that were nearly homologous. In this case, the collapse time $\tau_{\text {coll }}$ is roughly the free-fall time at the horizon

$$
\tau_{\text {coll }}=\left(\frac{R^{3}}{4 \pi M}\right)^{1 / 2}=14 \mathrm{~s}\left(\frac{M}{10^{6} M_{\odot}}\right)^{-1} .
$$

The peak GW frequency $f_{\mathrm{GW}}=\tau_{\text {coll }}^{-1}$ is then $10^{-2} \mathrm{~Hz}$, if the mass of the star is $10^{6} M_{\odot}$. This is in the middle of LISA's frequency band of $10^{-4}-1 \mathrm{~Hz}[243,76]$.

The amplitude $h$ of this burst signal can be roughly estimated in terms of the star's quadrupole moment

$$
\begin{aligned}
h & \leq \epsilon \frac{2 M^{2}}{R d} \\
& \leq \epsilon \cdot 1 \times 10^{-18}\left(\frac{M}{10^{6} M_{\odot}}\right)\left(\frac{d}{50 \mathrm{Gpc}}\right)^{-1} .
\end{aligned}
$$

Here $d$ is the distance to the star and $\epsilon \sim T /|W|$ is a measure of the star's deviation from spherical symmetry. In this case, $\epsilon$ will be much less than one near the horizon, since the collapse is nearly spherical. 
There are two possible aspherical collapse outcomes that can be discussed. The first outcome is direct collapse to a SMBH. In this case, $\epsilon$ will be on the order of one near the horizon. Thus, according to Equation 8, the peak amplitude of the GW burst signal will be

$$
h_{\mathrm{pk}} \sim 1 \times 10^{-18}\left(\frac{M}{10^{6} M_{\odot}}\right)\left(\frac{d}{50 \mathrm{Gpc}}\right)^{-1} .
$$

Alternatively, the star may encounter the dynamical bar mode instability prior to complete collapse. Baumgarte and Shapiro [16] have estimated that a uniformly rotating SMS will reach $\beta \sim 0.27$ when $R / M=15$. The frequency of the quasiperiodic gravitational radiation emitted by the bar can be estimated in terms of its rotation frequency to be

$$
\begin{aligned}
f_{\mathrm{GW}} & =2 f_{\mathrm{bar}} \sim 2\left(\frac{G M}{R^{3}}\right)^{1 / 2} \\
& =2 \times 10^{-3} \mathrm{~Hz}\left(\frac{M}{10^{6} M_{\odot}}\right)^{-1},
\end{aligned}
$$

when $R / M=15$. The corresponding $h_{\mathrm{pk}}$, again estimated in terms of the star's quadrupole moment, is

$$
\begin{aligned}
h_{\mathrm{pk}} & \leq \frac{2 M^{2}}{R d} \\
& \leq 1 \times 10^{-19}\left(\frac{M}{10^{6} M_{\odot}}\right)\left(\frac{d}{50 \mathrm{Gpc}}\right)^{-1} .
\end{aligned}
$$

The LISA sensitivity curve is shown in Figure 18 (see [119] for details on the computation of this curve; a mission time of 3 years has been assumed). The GW signal from this dynamical bar-mode could be detected with LISA.

Shibata and Shapiro [228] have published a fully general relativistic, axisymmetric simulation of the collapse of a rapidly, rigidly rotating SMS. They found that the collapse remained homologous during the early part of the evolution. An apparent horizon does appear in their simulation, indicating the formation of a black hole. Because of the symmetry condition used in their run, non-axisymmetric instabilities were unable to develop.

The collapse of a uniformly rotating SMS has been investigated with post-Newtonian hydrodynamics, in 3+1 dimensions, by Saijo, Baumgarte, Shapiro, and Shibata [208]. Their numerical scheme used a post-Newtonian approximation to the Einstein equations, but solved the fully relativistic hydrodynamics equations. Their initial model was an $n=3$ polytrope.

The results of Saijo et al. (confirmed in conformally flat simulations [207]) indicate that the collapse of a uniformly rotating SMS is coherent (i.e., no fragmentation instability develops). The collapse evolution of density contours from their model is shown in Figure 19. Although the work of Baumgarte and Shapiro [16] suggests that a bar instability should develop prior to BH formation, no bar development was observed by Saijo et al. They use the quadrupole approximation to estimate a mean GW amplitude from the collapse itself: $h=4 \times 10^{-21}$, for a $10^{6} M_{\odot}$ star located at a distance of $50 \mathrm{Gpc}$. Their estimate for $f_{\mathrm{GW}}$ at the time of $\mathrm{BH}$ formation is $3 \times 10^{-3} \mathrm{~Hz}$. This signal would be detectable with LISA (see Figure 18).

Saijo et al. also consider the GW emission from the ringdown of the black hole remnant. For the $l=m=2$ quasi-normal mode of a Kerr black hole with $a / M=0.9$, they estimate the characteristic amplitude of emission to be $h \sim 1.2 \times 10^{-20}\left[\left(\triangle E_{\mathrm{GW}} / M\right) / 10^{-4}\right]^{1 / 2}$ at $f_{\mathrm{GW}} \sim 2 \times 10^{-2} \mathrm{~Hz}$ for an $M=10^{6} M_{\odot}$ source located at a luminosity distance of $50 \mathrm{Gpc}$ (see $[149,242,229]$ for details). Here, $\triangle E_{\mathrm{GW}} / M$ is the radiated energy efficiency and may be $\lesssim 7 \times 10^{-4}$ [233]. This GW signal is within LISA's range of sensitivity (see Figure 18).

Living Reviews in Relativity

http: //www. livingreviews . org//rr-2003-2 


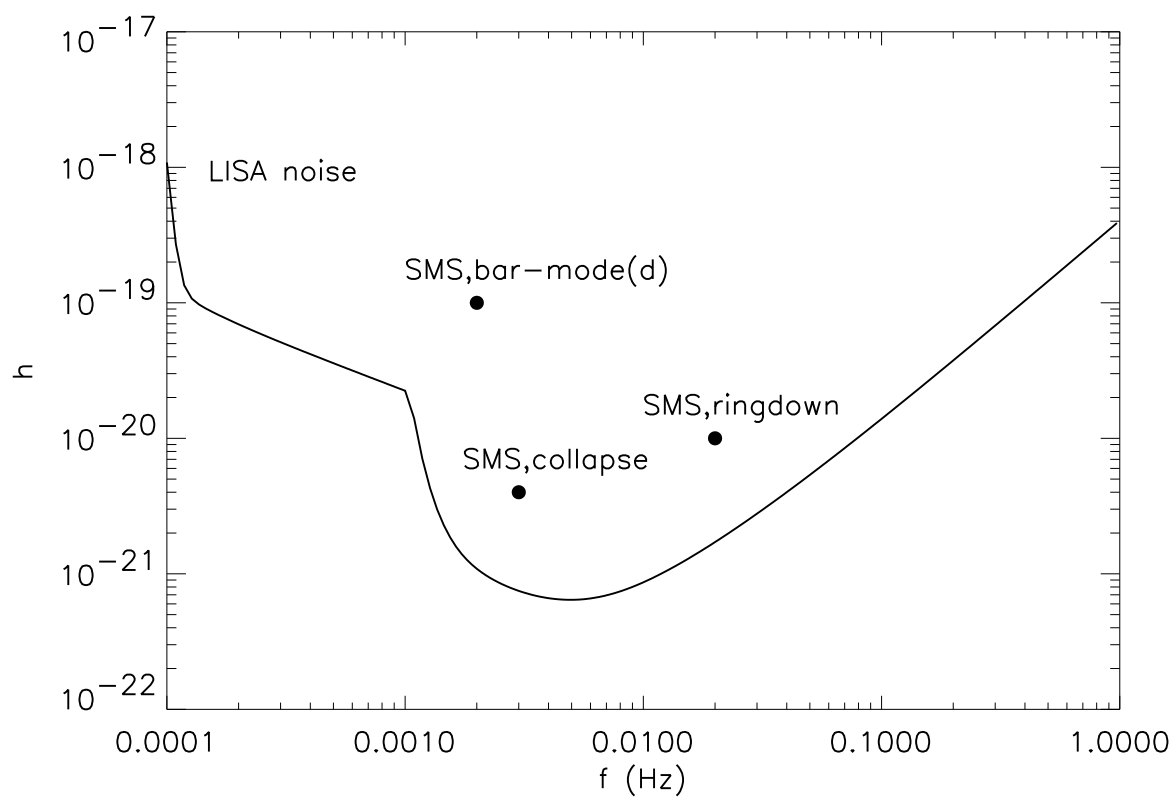

Figure 18: A comparison between the $G W$ amplitude $h(f)$ for various sources and the LISA noise curve. See the text for details regarding the computations of $h$. The SMS sources are assumed to be located at a luminosity distance of $50 \mathrm{Gpc}$. The bar-mode source is a dynamical bar-mode.

\section{Summary}

It is hoped that as gravitational collapse simulations become more sophisticated, the historically widely varying estimates of the magnitude of GW emission from collapse may start to converge. Steady progress in this field has been made in the last decade. Some researchers have begun to use progenitor models produced with stellar evolution codes, which thus have more realistic angular momentum profiles, as starting points for collapse simulations [86]. This reduces the need for collapse studies that include large surveys of the angular momentum parameter space. Other progress made in the numerical study of collapse includes the use of realistic equations of state [86], advanced neutrino transport and interaction schemes [130, 150, 197, 240], and the performance of 3D Newtonian [198, 35, 91, 92] and improved methods for general relativistic simulations [222, 61].

There is still much work to be done toward the goal of self-consistent, 3D general relativistic collapse simulations. The most rapidly rotating progenitors (with possibly the strongest GW signals) may be produced only in specific binary systems [95, 84, 265]. If the GW signal is dominated by convection, then it is critical that scientists actually understand the true supernova mechanism. Accurate progenitor modelling and collapse simulations must include the effects of magnetic fields, as they can significantly alter the amount of angular momentum and differential rotation present in collapsing stars. Many of the more advanced studies, which include proper microphysics treatment and/or general relativistic effects, have been limited to axisymmetry. Full 3D simulations are necessary to compute the characteristics of the GW emission from non-axisymmetric collapse phenomena. Furthermore, simulations that follow both the collapse and the evolution of the collapsed remnant are necessary to consistently predict GW emission. One benefit of long duration simulations is that they will facilitate the investigation of the effects of the envelope on any instabilities that develop in the collapsing core or remnant. Of course, lengthy 3D simulations are computationally intensive. This burden may be reduced by the use of advanced numerical 


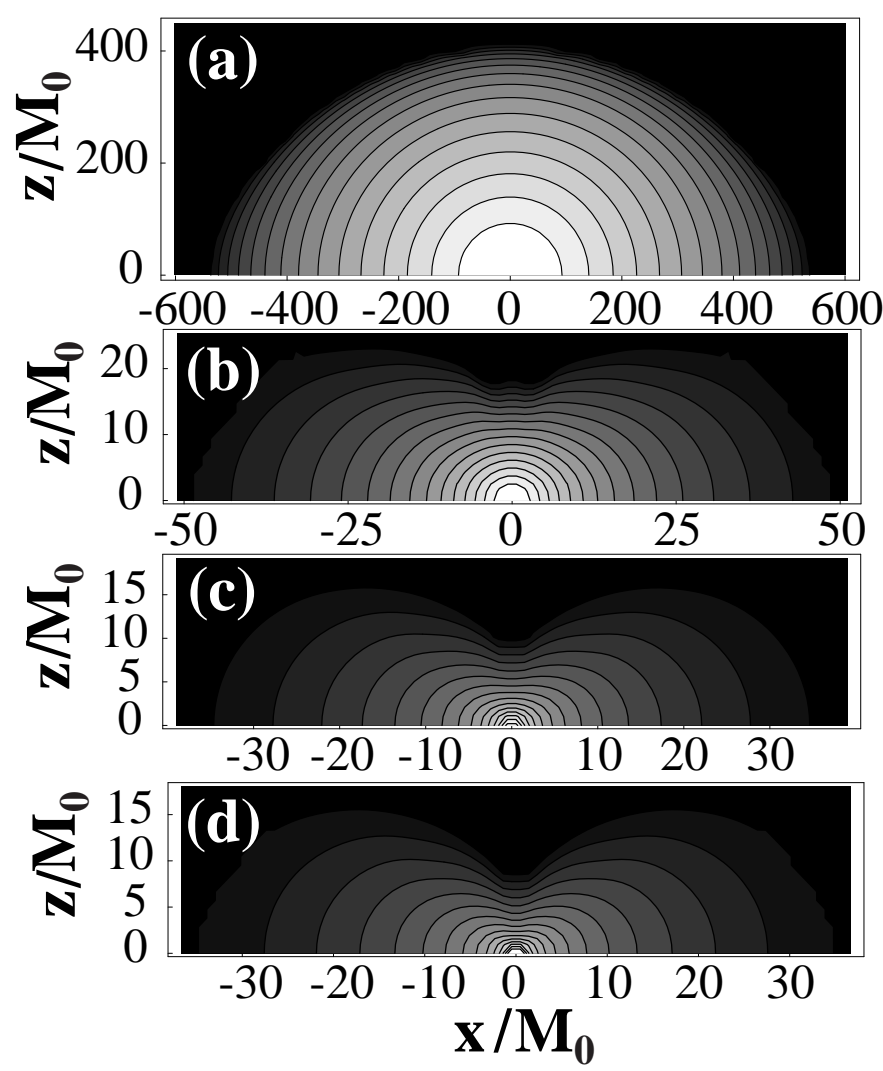

Figure 19: Meridional plane density contours from the SMS collapse simulation of Saijo, Baumgarte, Shapiro, and Shibata [208]. The contour lines denote densities $\rho=\rho_{\mathrm{c}} \times d^{(1-i / 16)}$, where $\rho_{\mathrm{c}}$ is the central density. The frames are plotted at $\left(t / t_{\mathrm{D}}, \rho_{\mathrm{c}}, d\right)=(a)\left(5.0628 \times 10^{-4}, 8.254 \times 10^{-9}\right.$, $\left.10^{-7}\right),(b)\left(2.50259,1.225 \times 10^{-4}, 10^{-5}\right),(c)\left(2.05360,8.328 \times 10^{-3}, 5.585 \times 10^{-7}\right),(d)(2.50405$, $\left.3.425 \times 10^{-2}, 1.357 \times 10^{-7}\right)$, respectively. Here $t, t_{\mathrm{D}}$, and $M_{0}$ are the time, dynamical time $\left(=\sqrt{R_{\mathrm{e}}^{3} / M}\right.$, where $R_{\mathrm{e}}$ is the initial equatorial radius and $M$ is the mass), and rest mass. (Figure 15 of [208]; used with permission.) 
techniques, including adaptive mesh refinement and parallel algorithms.

The current numerical simulations of gravitational collapse indicate that interferometric observatories could detect GWs emitted by some collapse phenomena. LIGO-I may be able to detect GWs from secular bar-mode instabilities in core collapse SNe [146] and magnetized tori surrounding black hole collapse remnants [251]. LIGO-II could observe GWs from dynamical bar-mode instabilities in AIC [155] and core collapse SNe [86]. LISA should be able to detect the collapse (and any bar-mode instabilities that develop during the collapse) of SMSs [16] and the ringdown of black hole remnants of collapsed Population III stars [86] and SMSs [208]. These observations will provide unique information about gravitational collapse, the supernova and gamma-ray burst explosion mechanisms, and their associated progenitors and remnants. 


\section{Acknowledgements}

It is a pleasure to thank Paul Bradley, Adam Burrows, Harald Dimmelmeier, Alex Heger, Scott Hughes, Hui Li, Ewald Müller, Ken Nomoto, Shangli Ou, and Stuart Shapiro for helpful conversations and/or permission to reprint figures/movies from their published works. We also gratefully acknowledge Ewald Müller and a second referee for their beneficial reviews of this article's first version. This work was performed under the auspices of the U.S. Department of Energy by the Los Alamos National Laboratory under contract W-7405-ENG-36.

Living Reviews in Relativity

http: //www . livingreviews . org/lrr-2003-2 


\section{References}

[1] Abel, T., Bryan, G.L., and Norman, M.L., "The Formation and Fragmentation of Primordial Molecular Clouds", Astrophys. J., 540, 39-44, (2000). Related online version (cited on 7 February 2000): http://arXiv.org/abs/astro-ph/0002135. 5.1, 5.2, 6.1

[2] Abel, T., Bryan, G.L., and Norman, M.L., "The formation of the first star in the universe", Science, 295, 93-98, (2002). Related online version (cited on 4 December 2001): http://arXiv.org/abs/astro-ph/0112088. 5.1, 5.2

[3] Akiyama, S., and Wheeler, J.C., "Magnetic Fields in Supernovae", in Fryer, C.L., ed., Stellar Collapse, Proceedings of "Core Collapse of Massive Stars", 200th AAS meeting, Albuquerque, NM, June 2002, vol. 302 of Astrophysics and Space Science Library, (Kluwer Academic Publishers, Dordrecht, Netherlands; Boston, U.S.A., 2004). Related online version (cited on 21 December 2005): http://arXiv.org/abs/astro-ph/0211458. 3.1, 3.4.4

[4] Alcubierre, M., Brügmann, B., Holz, D.E., Takahashi, R., Brandt, S., Seidel, E., and Thornburg, J., "Symmetry without symmetry: Numerical simulation of axisymmetric systems using Cartesian grids", Int. J. Mod. Phys. D, 10, 273-289, (2001). Related online version (cited on 4 August 1999):

http://arXiv.org/abs/gr-qc/9908012. 4.4

[5] Andersson, N., "A new class of unstable modes of rotating relativistic stars", Astrophys. J., 502, 708-713, (1998). Related online version (cited on 24 June 1997): http://arXiv.org/abs/gr-qc/9706075. 2.3

[6] Andersson, N., "Gravitational waves from instabilities in relativistic stars", Class. Quantum Grav., 20, R105-R144, (2002). URL (cited on 4 November 2002):

http: //arXiv.org/abs/astro-ph/0211057. 2.3

[7] Arnaud, N., Barsuglia, M., Bizouard, M.-A., Brisson, V., Cavalier, F., Davier, M., Hello, P., Kreckelbergh, S., and Porter, E., "Detection of a close supernova gravitational wave burst in a network of interferometers, neutrino and optical detectors", Astropart. Phys., 21, 201-221, (2004). 1

[8] Arnett, W.D., Bahcall, J. N., Kirshner, R.P., and Woosley, S.E., "Supernova 1987a", Annu. Rev. Astron. Astrophys., 27, 629-700, (1989). 3.1

[9] Arras, P., Flanagan, É.É., Morsink, S.M., Schenk, A.K., Teukolsky, S.A., and Wasserman, I., "Saturation of the $r$-mode instability", Astrophys. J., 591, 1129-1151, (2002). Related online version (cited on 21 December 2005): http://arXiv.org/abs/astro-ph/0202345. 2.3

[10] Baraffe, I., Heger, A., and Woosley, S.E., "On the stability of very massive supermassive stars", Astrophys. J., 550, 890-896, (2001). Related online version (cited on 26 September 2000):

http://arXiv.org/abs/astro-ph/0009410. 5.1

[11] Bardeen, M., and Piran, T., "General relativistic axisymmetric rotating systems: Coordinates and equations", Phys. Rep., 96, 205-250, (1983). 3.4.1

[12] Baron, E., Cooperstein, J., and Kahana, S., "Supernovae and the nuclear equation of state at high densities", Nucl. Phys. A, 440, 744-754, (1985). 3.1 
[13] Baron, E., Cooperstein, J., and Kahana, S., "Type II supernovae in $12 M_{\odot}$ and $15 M_{\odot}$ stars: The equation of state and general relativity", Phys. Rev. Lett., 55, 126-129, (1985). 3.1

[14] Baron, E., Cooperstein, J., Kahana, S., and Nomoto, K., "Collapsing white dwarfs", Astrophys. J., 320, 304-307, (1987). 2.4.1

[15] Baumgarte, T.W., Janka, H.-T., Keil, W., Shapiro, S.L., and Teukolsky, S.A., "Delayed Collapse of Hot Neutron Stars to Black Holes via Hadronic Phase Transitions", Astrophys. J., 468, 823-833, (1996). 4.5

[16] Baumgarte, T.W., and Shapiro, S.L., "Evolution of rotating supermassive stars to the onset of collapse", Astrophys. J., 526, 941-952, (1999). Related online version (cited on 14 September 1999):

http://arXiv.org/abs/astro-ph/9909237. 2.4.2, 6.1, 6.4, 6.4, 7

[17] Baumgarte, T.W., and Shapiro, S.L., "Collapse of a Magnetized Star to a Black Hole", Astrophys. J., 585, 930-947, (2003). URL (cited on 14 November 2002): http://arXiv.org/abs/astro-ph/0211339. 3.1

[18] Baumgarte, T.W., Shapiro, S.L., and Teukolsky, S.A., "Computing supernova collapse to neutron stars and black holes", Astrophys. J., 443, 717-734, (1995). 4.5

[19] Baumgarte, T.W., Shapiro, S.L., and Teukolsky, S.A., "Computing the Delayed Collapse of Hot Neutron Stars to Black Holes", Astrophys. J., 458, 680-691, (1996). 4.5

[20] Bazan, G., and Arnett, D., "Convection, nucleosynthesis, and core collapse", Astrophys. J. Lett., 433, L41-L43, (1994). 3.4.5

[21] Begelman, M.C., and Rees, M.J., "The fate of dense stellar systems", Mon. Not. R. Astron. Soc., 185, 847-860, (1978). 6.1

[22] Berger, E., Kulkarni, S.R., Frail, D.A., and Soderberg, A.M., "A Radio Survey of Type Ib and Ic Supernovae: Searching for Engine-driven Supernovae", Astrophys. J., 599, 408-418, (2003). $\quad 4.2$

[23] Bethe, H.A., "Supernova mechanisms", Rev. Mod. Phys., 62, 801-866, (1990). 3.1

[24] Bethe, H.A., and Wilson, J.R., "Revival of a stalled supernova shock by neutrino heating", Astrophys. J., 295, 14-23, (1985). 3.1

[25] Blanchet, L., Damour, T., and Schäfer, G., "Post-Newtonian hydrodynamics and postNewtonian gravitational wave generation for numerical relativity", Mon. Not. R. Astron. Soc., 242, 289-305, (1990). 2.4.1

[26] Blondin, J.M., Mezzacappa, A., and DeMarino, C., "Stability of Standing Accretion Shocks, with an Eye toward Core-Collapse Supernovae", Astrophys. J., 584, 971-980, (2003). 3.1, 3.4 .5

[27] Bodenheimer, P., and Ostriker, J.P., "Rapidly rotating stars. VIII. Zero-viscosity polytropic sequences", Astrophys. J., 180, 159-170, (1973). 6.1

[28] Bonazzola, S., and Marck, J.-A., "Efficiency of gravitational radiation from axisymmetric and 3D stellar collapse. I - Polytropic case", Astron. Astrophys., 267, 623-633, (1993). 3.4 .3

[29] Bowers, R.L., and Wilson, J.R., "Collapse of iron stellar cores", Astrophys. J., 263, 366-376, (1982). 3.1 
[30] Bowers, R.L., and Wilson, J.R., "A numerical model for stellar core collapse", Astrophys. J. Suppl. Ser., 50, 115-159, (1982). 3.1

[31] Brachwitz, F., Dean, D.J., Hix, W.R., Iwamoto, K., Langanke, K., Martínez-Pinedo, G., Nomoto, K., Strayer, M.R., Thielemann, F.-K., and Umeda, H., "The role of electron captures in Chandrasekhar-mass models for Type Ia supernovae", Astrophys. J., 536, 934-947, (2000). Related online version (cited on 26 January 2000): http://arXiv.org/abs/astro-ph/0001464. 2.1

[32] Bravo, E., and García-Senz, D., "Coulomb corrections to the equation of state of nuclear statistical equilibrium matter: Implications for SNIa nucleosynthesis and the accretion-induced collapse of white dwarfs", Mon. Not. R. Astron. Soc., 307, 984-992, (1999). 2.1

[33] Bromm, V., Coppi, P.S., and Larson, R.B., "Forming the first stars in the universe: The fragmentation of primordial gas", Astrophys. J. Lett., 527, L5-L8, (1999). Related online version (cited on 13 October 1999):

http://arXiv.org/abs/astro-ph/9910224. 6.1

[34] Brown, J.D., "Gravitational waves from the dynamical bar instability in a rapidly rotating star", Phys. Rev. D, 62, 084024-1-11, (2000). Related online version (cited on 1 April 2000): http://arXiv.org/abs/gr-qc/0004002. 2.4.2

[35] Brown, J.D., "Rotational instabilities in post-collapse stellar cores", in Centrella, J.M., ed., Astrophysical Sources for Ground-Based Gravitational Wave Detectors, Philadelphia, Pennsylvania, 30 October - 1 November 2000, AIP Conference Proceedings, 234-245, (American Institute of Physics, Melville, U.S.A., 2001). 3.4.3, 7

[36] Bruenn, S.W., "Numerical simulations of core collapse supernovae", in Guidry, M.W., and Strayer, M.R., eds., Nuclear Physics in the Universe, Proceedings of the First Symposium on Nuclear Physics in the Universe held in Oak Ridge, Tennessee, USA, 24-26 September 1992, 31-50, (Institute of Physics, Bristol, U.K.; Philadelphia, U.S.A., 1993). 3.1

[37] Bruenn, S.W., De Nisco, K.R., and Mezzacappa, A., "General relativistic effects in the core collapse supernova mechanism", Astrophys. J., 560, 326-338, (2001). Related online version (cited on 23 January 2001): http://arXiv.org/abs/astro-ph/0101400. 3.4 .4

[38] Buonanno, A., Sigl, G., Raffelt, G.G., Janka, H.-T., and Müller, E., "Stochastic gravitationalwave background from cosmological supernovae", Phys. Rev. D, 72, 084001, (2005). 3.5

[39] Buras, R., Rampp, M., Janka, H.-T., and Kifonidis, K., "Improved Models of Stellar Core Collapse and Still No Explosions: What Is Missing?", Phys. Rev. Lett., 90, 241101-1-4, (2003). 3.1

[40] Burrows, A., personal communication, (2005). 3.4.5

[41] Burrows, A., and Hayes, J., "Pulsar recoil and gravitational radiation due to asymmetrical stellar collapse and explosion", Phys. Rev. Lett., 76, 352-355, (1996). 3.1, 3.3, 3.4.5, 11

[42] Burrows, A., Hayes, J., and Fryxell, B.A., "On the Nature of Core-Collapse Supernova Explosions", Astrophys. J., 450, 830-850, (1995). 3.1

[43] Burrows, A., Livne, E., Dessart, L., Ott, C., and Murphy, J., "A New Mechanism for CoreCollapse Supernova Explosions", Astrophys. J., accepted, (2005). Related online version (cited on 21 December 2005):

http://arXiv.org/abs/astro-ph/0510687. 3.1, 3.4.5 
[44] Burrows, A., and Thompson, T.A., "Neutrino-Matter Interaction Rates in Supernovae", in Fryer, C.L., ed., Stellar Collapse, Proceedings of "Core Collapse of Massive Stars", 200th AAS meeting, Albuquerque, NM, June 2002, vol. 302 of Astrophysics and Space Science Library, (Kluwer Academic Publishers, Dordrecht, Netherlands; Boston, U.S.A., 2004). Related online version (cited on 21 December 2005):

http://arXiv.org/abs/astro-ph/0211404. 3.1

[45] California Institute of Technology, "LIGO Laboratory Home Page", project homepage. URL (cited on 4 October 2002):

http://www.ligo.caltech.edu. 1

[46] Cappellaro, E., Evans, R., and Turatto, M., "A new determination of supernova rates and a comparison with indicators for galactic star formation", Astron. Astrophys., 351, 459-466, (1999). 3.2

[47] Carr, B.J., Bond, J.R., and Arnett, W.D., "Cosmological consequences of population III stars", Astrophys. J., 277, 445-469, (1984). 5.2, 5.4

[48] Cassisi, S., Iben Jr, I., and Tornambé, A., "Hydrogen-accreting carbon-oxygen white dwarfs", Astrophys. J., 496, 376-385, (1998). 2.2

[49] Centrella, J.M., New, K.C.B., Lowe, L., and Brown, J.D., "Dynamical rotational instability at low T/W", Astrophys. J. Lett., 550, L193-L196, (2001). Related online version (cited on 27 October 2000):

http://arXiv.org/abs/astro-ph/0010574. 2.3, 2.4.2, 3.4.3

[50] Chandrasekhar, S., "Dynamical Instability of Gaseous Masses Approaching the Schwarzschild Limit in General Relativity", Phys. Rev. Lett., 12, 114-116, (1964). Erratum 437-438. 6.1

[51] Chandrasekhar, S., "The dynamical instability of gaseous masses approaching the Schwarzschild limit in general relativity", Astrophys. J., 140, 417-433, (1964). 6.1

[52] Chandrasekhar, S., An Introduction to the Study of Stellar Structure, (Dover, New York, U.S.A., 1967). 2.4 .2

[53] Colgate, S.A., "Supernova: Hot bubbles drive explosions", Nature, 341, 489-490, (1989). 3.1

[54] Colgate, S.A., Herant, M., and Benz, W., "Neutron star accretion and the neutrino fireball", Phys. Rep., 227, 157-174, (1993). 3.1

[55] Colgate, S.A., and White, R.H., "The hydrodynamic behavior of supernovae explosions", Astrophys. J., 143, 626-681, (1966). 3.1

[56] Cook, G.B., Shapiro, S.L., and Teukolsky, S.A., "Testing a simplified version of Einstein's equations for numerical relativity", Phys. Rev. D, 53, 5533-5540, (1996). Related online version (cited on 5 December 1995):

http://arXiv.org/abs/astro-ph/9512009. 3.4.4

[57] Detweiler, S., and Lindblom, L., "On the evolution of the homogeneous ellipsoidal figures. II. Gravitational collapse and gravitational radiation", Astrophys. J., 250, 739-749, (1981). $1,3.4 .1$

Living Reviews in Relativity

http://www.livingreviews.org/lrr-2003-2 
[58] Dimmelmeier, H., Font, J.A., and Müller, E., "Gravitational waves from relativistic rotational core collapse", Astrophys. J. Lett., 560, L163-L166, (2001). Related online version (cited on 21 April 2001):

http://arXiv.org/abs/astro-ph/0103088. 3.4.4

[59] Dimmelmeier, H., Font, J.A., and Müller, E., "Relativistic simulations of rotational core collapse. I. Methods, initial models, and code tests", Astron. Astrophys., 388, 917-935, (2002). Related online version (cited on 17 April 2002):

http://arXiv.org/abs/astro-ph/0204288. 3.4.4

[60] Dimmelmeier, H., Font, J.A., and Müller, E., "Relativistic simulations of rotational core collapse. II. Collapse dynamics and gravitational radiation", Astron. Astrophys., 393, 523542, (2002). Related online version (cited on 17 April 2002):

http://arXiv.org/abs/astro-ph/0204289. 3.4.3, 3.4.4, 7, 8, 9, 10

[61] Dimmelmeier, H., Novak, J., Font, J.A., Ibáñez, J.M., and Müller, E., "Combining spectral and shock-capturing methods: A new numerical approach for 3D relativistic core collapse simulations", Astron. Astrophys., 560, 163-166, (2005). 7

[62] Duez, M.D., Shapiro, S.L., and You, H.-J., "Relativistic hydrodynamic evolutions with black hole excision", Phys. Rev. D, 69, 104016, (2004). 4.4

[63] Durisen, R.H., and Tohline, J.E., "Fission of rapidly rotating fluid systems", in Black, D., and Matthews, M., eds., Protostars and Planets II, 534-575, (University of Arizona Press, Tucson, U.S.A., 1985). 2.3

[64] Eisenstein, D.J., and Loeb, A., "Origin of quasar progenitors from the collapse of low-spin cosmological perturbations", Astrophys. J., 443, 11-17, (1995). Related online version (cited on 11 January 1994):

http://arXiv.org/abs/astro-ph/9401016. 6.1

[65] Epstein, R., The post-Newtonian theory of the generation of gravitational radiation and its application to stellar collapse, Ph.D. Thesis, (Stanford University, Stanford, U.S.A., 1976). 3.4.1

[66] Epstein, R., and Wagoner, R.V., "Post-Newtonian generation of gravitational waves", Astrophys. J., 197, 717-723, (1975). 3.4.1

[67] Eriguchi, Y., and Müller, E., "Equilibrium models of differentially rotating polytropes and the collapse of rotating stellar cores", Astron. Astrophys., 147, 161-168, (1985). 3.4 .2

[68] Ferrarese, L., and Merritt, D., "A fundamental relation between supermassive black holes and their host galaxies", Astrophys. J. Lett., 539, L9-L12, (2000). Related online version (cited on 4 June 2000): http://arXiv.org/abs/astro-ph/0006053. 6.1

[69] Ferrari, V., Matarrese, S., and Schneider, R., "Gravitational wave background from a cosmological population of core-collapse supernovae", Mon. Not. R. Astron. Soc., 303, 247-257, (1999). Related online version (cited on 15 October 1998):

http://arXiv.org/abs/astro-ph/9804259. 3.5

[70] Ferrari, V., Miniutti, G., and Pons, J.A., "Gravitational waves from newly born, hot neutron stars", Mon. Not. R. Astron. Soc., 342, 629-638, (2003). Related online version (cited on 26 October 2002):

http://arXiv.org/abs/astro-ph/0210581. 3.4.3 
[71] Finn, L.S., "Supernovae, gravitational radiation, and the quadrupole formula", in Evans, C.R., Finn, L.S., and Hobill, D.W., eds., Frontiers in Numerical Relativity, International workshop devoted to research in numerical relativity, held in Urbana-Champaign in May 1988, 126-145, (Cambridge University Press, Cambridge, U.K.; New York, U.S.A., 1989). $3.1,3.3$

[72] Finn, L.S., "Detectability of gravitational radiation from stellar-core collapse", in Buchler, J.R., Detweiler, S., and Ipser, J.R., eds., Nonlinear problems in relativity and cosmology, 6th Florida Workshop in Nonlinear Astronomy, held on October 2-4, 1990 in Gainesville, Florida, 156-172, (New York Academy of Sciences, New York, U.S.A., 1991). 3.4.1

[73] Finn, L.S., "Gravitional Radiation Sources and Signatures", in Dixon, L.J., ed., Gravity: From the Hubble Length to the Planck Length, Proceedings of the 26th SLAC Summer Institute on Particle Physics (SSI 98), Stanford, USA, 3-14 August 1998, vol. 538 of SLAC-R, (SLAC, Springfield, U.S.A., 2001). URL (cited on 30 March 1999):

http://www.slac.stanford.edu/pubs/confproc/ssi98/ssi98-007.html. also at: http://arXiv.org/abs/gr-qc/9903107. 1

[74] Finn, L.S., and Evans, C.R., "Determining gravitational radiation from Newtonian selfgravitating systems", Astrophys. J., 351, 588-600, (1990). 1, 2.4.1, 3.4.2

[75] Foglizzo, T., Scheck, L., and Janka, H.-T., "Neutrino-driven convection versus advection in core collapse supernovae", Astron. Astrophys., submitted, (2005). Related online version (cited on 21 December 2005):

http://arXiv.org/abs/astro-ph/0507636. 3.1

[76] Folkner, W.M., ed., Laser Interferometer Space Antenna: Second International LISA Symposium on the Detection and Observation of Gravitational Waves in Space, Pasadena 1998, vol. 456 of AIP Conference Proceedings, (Springer, New York, U.S.A., 1993). 6.4

[77] Friedman, J.L., and Morsink, S.M., "Axial instability of rotating relativistic stars", Astrophys. J., 502, 714-720, (1998). Related online version (cited on 23 June 1997): http://arXiv.org/abs/gr-qc/9706073. 2.3

[78] Fryer, C.L., "Mass Limits For Black Hole Formation", Astrophys. J., 522, 413-418, (1999). 3.1, 4.1

[79] Fryer, C.L., "Stellar Collapse", Int. J. Mod. Phys. D, 12, 1795-1835, (2003). 3.1

[80] Fryer, C.L., "Neutron Star Kicks from Asymmetric Collapse", Astrophys. J., 601, L175L178, (2004). 3.1, 3.3, 3.4.3, 3.4.5

[81] Fryer, C.L., ed., Stellar Collapse, Proceedings of "Core Collapse of Massive Stars", 200th AAS meeting, Albuquerque, NM, June 2002, vol. 302 of Astrophysics and Space Science Library, (Kluwer Academic Publishers, Dordrecht, Netherlands; Boston, U.S.A., 2004). 3.1

[82] Fryer, C.L., Benz, W., Herant, M., and Colgate, S.A., "What can the accretion-induced collapse of white dwarfs really explain?", Astrophys. J., 516, 892-899, (1999). Related online version (cited on 2 December 1998):

http: //arXiv.org/abs/astro-ph/9812058. 2.1, 2.2, 2.4.1, 2.4.1, 2.4.1, 2.4.1, 2.4.2

[83] Fryer, C.L., and Heger, A., "Core-Collapse Simulations of Rotating Stars", Astrophys. J., 541, 1033-1050, (2000). Related online version (cited on 30 July 1999): http://arXiv.org/abs/astro-ph/9907433. 3.1, 3.4.3, 3.4.4 
[84] Fryer, C.L., and Heger, A., "Binary Merger Progenitors for Gamma-Ray Bursts and Hypernovae", Astrophys. J., 623, 302-313, (2005). 7

[85] Fryer, C.L., Heger, A., Langer, N., and Wellstein, S., "Stellar Collapse", Int. J. Mod. Phys. $D, \mathbf{1 2}, 1795-1835,(2003) . \quad 3.1$

[86] Fryer, C.L., Holz, D.E., and Hughes, S.A., "Gravitational wave emission from core-collapse of massive stars", Astrophys. J., 565, 430-446, (2002). Related online version (cited on 25 September 2001):

http://arXiv.org/abs/astro-ph/0106113. 1, 2.4.1, 2.4.1, 3.1, 3.4.4, 4.4, 5.2, 5.4, 7

[87] Fryer, C.L., Holz, D.E., and Hughes, S.A., "Gravitational Waves from Stellar Collapse: Correlations to Explosion Asymmetries", Astrophys. J., 609, 288-300, (2004). 1, 3.4.3, 12 , $13,3.4 .5$

[88] Fryer, C.L., Holz, D.E., Hughes, S.A., and Warren, M.S., "Stellar Collapse and Gravitational Waves", in Fryer, C.L., ed., Stellar Collapse, Proceedings of "Core Collapse of Massive Stars", 200th AAS meeting, Albuquerque, NM, June 2002, vol. 302 of Astrophysics and Space Science Library, (Kluwer Academic Publishers, Dordrecht, Netherlands; Boston, U.S.A., 2004). Related online version (cited on 21 December 2005):

http://arXiv.org/abs/astro-ph/0211609. 1, 3.4.3, 3.4.4, 3.4.4, 5.4

[89] Fryer, C.L., and Kalogera, V., "Theoretical black hole mass distributions", Astrophys. J., $\mathbf{5 5 4}, 548-560,(2001) . \quad 3.1,3.2,4.2$

[90] Fryer, C.L., and Kusenko, A., "Effects of neutrino-driven kicks on the supernova explosion mechanism", Astrophys. J., submitted, (2005). Related online version (cited on 21 December 2005):

http://arXiv.org/abs/astro-ph/0512033. 3.4.3

[91] Fryer, C.L., and Warren, M.S., "Modeling Core-Collapse Supernovae in Three Dimensions", Astrophys. J. Lett., 574, L65-L68, (2002). URL (cited on 3 June 2002):

http://arXiv.org/abs/astro-ph/0206017. 1, 3.1, 3.4.3, 7

[92] Fryer, C.L., and Warren, M.S., "The Collapse of Rotating Massive Stars in Three Dimensions", Astrophys. J., 601, 391-404, (2004). 3.1, 3.4.3, 7

[93] Fryer, C.L., and Woosley, S.E., "Helium Star/Black Hole Mergers: A New Gamma-Ray Burst Model", Astrophys. J., 502, L9-L12, (1998). 4.2

[94] Fryer, C.L., Woosley, S.E., and Hartmann, D., "Formation Rates of Black Hole Accretion Disk Gamma-Ray Bursts", Astrophys. J., 526, 152-177, (1999). 4.2

[95] Fryer, C.L., Woosley, S.E., and Hartmann, D.H., "Formation Rates of Black Hole Accretion Disk Gamma-Ray Bursts", Astrophys. J., 526, 152-177, (1999). 7

[96] Fryer, C.L., Woosley, S.E., and Heger, A., "Pair-instability supernovae, gravity waves, and gamma-ray transients", Astrophys. J., 550, 372-382, (2001). Related online version (cited on 13 July 2000):

http://arXiv.org/abs/astro-ph/0007176. 2.3, 5.1, 5.2, 5.4

[97] Gressman, P., Lin, L.-M., Suen, W.-M., Stergioulas, N., and Friedman, J.L., "Nonlinear $r$-modes in neutron stars: Instability of an unstable mode", Phys. Rev. D, 66, 041303-1-5, (2002). $\quad 2.3$ 
[98] Hachisu, I., "A versatile method for obtaining structures of rapidly rotating stars", Astrophys. J. Suppl. Ser., 61, 479-507, (1986). 2.4 .2

[99] Haehnelt, M.G., "Low-frequency gravitational waves from supermassive black holes", Mon. Not. R. Astron. Soc., 269, 199-208, (1994). 6.2

[100] Haehnelt, M.G., Natarajan, P., and Rees, M.J., "High-redshift galaxies, their active nuclei and central black holes", Mon. Not. R. Astron. Soc., 300, 817-827, (1998). Related online version (cited on 18 December 1997):

http://arXiv.org/abs/astro-ph/9712259. 6.1

[101] Haehnelt, M.G., and Rees, M.J., "The formation of nuclei in newly formed galaxies and the evolution of the quasar population", Mon. Not. R. Astron. Soc., 263, 168-178, (1993). 6.1

[102] Haensel, P., Levenfish, K.P., and Yakovlev, D.G., "Bulk viscosity in superfluid neutron star cores. III. Effects of $\Sigma^{-}$hyperons", Astron. Astrophys., 381, 1080-1089, (2002). Related online version (cited on 26 October 2001):

http://arXiv.org/abs/astro-ph/0110575. 2.3

[103] Hamuy, M., "Observed and physical properties of core-collapse supernovae", Astrophys. J., 582, 905-914, (2003). URL (cited on 10 September 2002): http://arXiv.org/abs/astro-ph/0209174. 3.1

[104] Hannover University, "GEO 600 home page", project homepage. URL (cited on 4 October 2002):

http://www.geo600.uni-hannover.de/. 1

[105] Hayashi, A., Eriguchi, Y., and Hashimoto, M., "On the possibility of the nonexplosive core contraction of massive stars: New evolutionary paths from rotating white dwarfs to rotating neutron stars", Astrophys. J., 492, 286-297, (1998). 3.4 .2

[106] Hayashi, A., Eriguchi, Y., and Hashimoto, M., "On the possibility of the nonexplosive core contraction of massive stars. II. General relativistic analysis", Astrophys. J., 521, 376-381, (1999). $\quad 3.4 .2$

[107] Heger, A., The presupernova evolution of rotating massive stars, Ph.D. Thesis, (Technische Universität München, Munich, Germany, 1998). 3.4.4, 5.4

[108] Heger, A., Fryer, C.L., Woosley, S.E., Langer, N., and Hartmann, D.H., "How Massive Single Stars End Their Life", Astrophys. J., 591, 288-300, (2001). 3.1

[109] Heger, A., Langer, N., and Woosley, S.E., "Presupernova evolution of rotating massive stars. I. Numerical method and evolution of the internal stellar structure", Astrophys. J., 528, 368-396, (2000). Related online version (cited on 12 April 1999): http://arXiv.org/abs/astro-ph/9904132. 3.4.3

[110] Heger, A., Woosley, S.E., and Spruit, H.C., "Presupernova Evolution of Differentially Rotating Massive Stars Including Magnetic Fields", Astrophys. J., 626, 350-363, (2005). 3.4 .4

[111] Herant, M., "Inside the Supernova: A Powerful Convective Engine", Phys. Rep., 256, 117133, (1995). $3.4 .5,3.4 .5$

[112] Herant, M., Benz, W., Hiz, W.R., Fryer, C.L., and Colgate, S.A., "Inside the Supernova: A Powerful Convective Engine", Astrophys. J., 435, 339-361, (1994). 3.1

Living Reviews in Relativity

http: //www. livingreviews . org/lrr-2003-2 
[113] Hillebrandt, W., "Stellar Collapse and Supernova Explosions", in Pacini, F., ed., High Energy Phenomena around Collapsed Stars, Proceedings of the NATO Advanced Study Institute, Cargèse, Corsica, France, September 2-13, 1985, vol. 195 of NATO Science Series, 73-104, (Reidel, Dordrecht, Netherlands; Boston, U.S.A., 1987). 3.4.5

[114] Hillebrandt, W., Nomoto, K., and Wolff, R.G., "Supernova explosions of massive stars - The mass range 8 to 10 solar masses", Astron. Astrophys., 133, 175-184, (1984). 2.1

[115] Ho, W.C.G., and Lai, D., " $r$-Mode oscillations and spin-down of young rotating magnetic neutron stars", Astrophys. J., 543, 386-394, (2000). Related online version (cited on 15 December 1999):

http://arXiv.org/abs/astro-ph/9912296. 2.4.1

[116] Höflich, P., Khokhlov, A., Wang, L., Wheeler, J.C., and Baade, D., "Aspherical Supernovae Explosions", in van der Hucht, K.A., Herrero, A., and Esteban, C., eds., A Massive Star Odyssey, from Main Sequence to Supernova, Lanzarote, Canary Islands, Spain, June 24-28, 2002, vol. 212 of IAU Symposia, (Astronomical Society of the Pacific, San Francisco, U.S.A., 2003). Related online version (cited on 12 July 2002):

http://arXiv.org/abs/astro-ph/0207272. 3.1

[117] Hogan, C.J., "A model of pregalactic evolution", Mon. Not. R. Astron. Soc., 188, 781-790, (1979). $\quad 5.2$

[118] Houser, J.L., "The effect of rotation on the gravitational radiation and dynamical instability of stiff stellar cores", Mon. Not. R. Astron. Soc., 299, 1069-1086, (1998). 2.3

[119] Hughes, S.A., "Untangling the merger history of massive black holes with LISA", Mon. Not. R. Astron. Soc., 331, 805-816, (2002). Related online version (cited on 30 August 2001): http://arXiv.org/abs/astro-ph/0108483. 6.4

[120] Hughes, S.A., Márka, S., Bender, P.L., and Hogan, C.J., "New physics and astronomy with the new gravitational-wave observatories", in Graf, N., ed., Proceedings of Snowmass 2001, The Future of Particle Physics, 30 June - 21 July 2001, Snowmass Village, Colorado, vol. C010630, P402, (SLAC eConf, Stanford, U.S.A., 2001). URL (cited on 15 October 2001): http://www.slac.stanford.edu/econf/C010630/proceedings.shtml. 1

[121] Hungerford, A., Fryer, C.L., and Rockefeller, G., "Gamma Rays from Single-Lobe Supernova Explosions", Astrophys. J., 635, 487-501, (2005). 3.1

[122] Hungerford, A., Fryer, C.L., and Warren, M.S., "Gamma Rays from Asymmetric Supernovae", Astrophys. J., 594, 390-403, (2005). 3.1

[123] Iben, I., "Massive stars in quasi-static equilibrium", Astrophys. J., 138, 1090-1096, (1963). 6.1

[124] Imamura, J.N., and Durisen, R.H., "The Dominance of Dynamic Barlike Instabilities in the Evolution of a Massive Stellar Core Collapse That "Fizzles"”, Astrophys. J., 549, 1062-1075, (2001). $\quad 3.4 .2$

[125] Imamura, J.N., Friedman, J.L., and Durisen, R.H., "Secular stability limits for rotating polytropic stars", Astrophys. J., 294, 474-478, (1985). 2.3

[126] INFN, "The Virgo Project", project homepage. URL (cited on 4 October 2002): http://www.virgo.infn.it/. 1 
[127] Isern, J., Canal, R., and Labay, J., "The outcome of explosive ignition of ONeMg cores: supernovae, neutron stars, or "iron" white dwarfs?", Astrophys. J. Lett., 372, L83-L86, (1991). $\quad 2.1$

[128] Janka, H.-T., "Conditions for shock revival by neutrino heating in core-collapse supernovae", Astron. Astrophys., 368, 527-560, (2001). Related online version (cited on 28 August 2000): http://arXiv.org/abs/astro-ph/0008432. 3.1

[129] Janka, H.-T., "Supermassive Stars: Fact or Fiction?", in Chui, C.K., Siuniaev, R.A., and Churazov, E., eds., Lighthouses of the Universe: The Most Luminous Celestial Objects and Their Use for Cosmology, Proceedings of the MPA/ESO/MPE/USM Joint Astronomy Conference, held in Garching, Germany, 6-10 August 2001, ESO Astrophysics Symposia, 357368, (Springer, Berlin, Germany; New York, U.S.A., 2002). Related online version (cited on 1 February 2002):

http://arXiv.org/abs/astro-ph/0202028. 6.1

[130] Janka, H.-T., Buras, R., Kifonidis, K., Rampp, M., and Plewa, T., "Explosion Mechanisms of Massive Stars", in Fryer, C.L., ed., Stellar Collapse, Proceedings of "Core Collapse of Massive Stars", 200th AAS meeting, Albuquerque, NM, June 2002, vol. 302 of Astrophysics and Space Science Library, (Kluwer Academic Publishers, Dordrecht, Netherlands; Boston, U.S.A., 2004). Related online version (cited on 21 December 2005):

http://arXiv.org/abs/astro-ph/0212314. 3.1, 7

[131] Janka, H.-T., and Müller, E., "Neutrino-driven type-II supernovae: Neutrino heating and post bounce dynamics", in Suzuki, Y., and Nakamura, K., eds., Frontiers of Neutrino Astrophysics, Proceedings of the International Symposium on Neutrino Astrophysics held on October 19-22, 1992, Takayama / Kamioka, Japan, vol. 5 of Frontiers Science Series, 203217, (Universal Academy Press, Tokyo, Japan, 1993). 3.1

[132] Janka, H.-T., and Müller, E., "Dynamics of Type-II supernovae", in McCray, R., and Wang, Z., eds., Supernovae and Supernovae Remnants, Proceedings of the IAU Colloquium 145, held in Xian, China, May 24-29, 1993, 109-118, (Cambridge University Press, Cambridge, U.K., 1996). 3.1

[133] Janka, H.-T., and Müller, E., "Neutrino heating, convection, and the mechanism of Type-II supernova explosions", Astron. Astrophys., 306, 167-198, (1996). 3.1

[134] Jenet, F.A., and Prince, T.A., "Detection of variable frequency signals using a fast chirp transform", Phys. Rev. D, 62, 122001-1-10, (2000). Related online version (cited on 7 December 2000):

http://arXiv.org/abs/gr-qc/0012029. 3.4 .4

[135] Jones, P.B., "Bulk viscosity of neutron-star matter", Phys. Rev. D, 64, 084003-1-7, (2001). 2.3

[136] Jones, P.B., "Comment on "Gravitational radiation instability in hot young neutron stars"", Phys. Rev. Lett., 86, 1384, (2001). 2.3

[137] Kato, M., and Hachisu, I., "A new estimation of mass accumulation efficiency in helium shell flashes toward Type Ia supernova explosions", Astrophys. J. Lett., 513, L41-L44, (1999). Related online version (cited on 8 January 1999):

http://arXiv.org/abs/astro-ph/9901080. 2.2

Living Reviews in Relativity

http://www. livingreviews.org/lrr-2003-2 
[138] Kifonidis, K., Plewa, T., Scheck, L., Janka, H.-T., and Müller, E., "Non-Spherical CoreCollapse Supernovae II. Late-Time Evolution of Globally Anisotropic Neutrino-Driven Explosions and Implications for SN 1987A", Astron. Astrophys., submitted, (2005). Related online version (cited on 21 December 2005):

http://arXiv.org/abs/astro-ph/0511369. 3.1

[139] King, A.R., and Lasota, J.-P., "Spin evolution and magnetic fields in cataclysmic variables", Astrophys. J., 378, 674-681, (1991). 2.4 .1

[140] Kobayashi, S., and Mészáros, P., "Gravitational radiation from gamma-ray burst progenitors", Astrophys. J., 589, 861-870, (2003). Related online version (cited on 9 October 2002): http://arXiv.org/abs/astro-ph/0210211. 4.3

[141] Kobayashi, S., and Mészáros, P., "Polarized Gravitational Waves from Gamma-Ray Bursts", Astrophys. J., 585, L89-L92, (2003). 4.3

[142] Kormendy, J., "Supermassive Black Holes in Disk Galaxies", in Funes, J.G., and Corsini, E.M., eds., Galaxy Disks and Disk Galaxies, Proceedings of a conference sponsored by the Vatican Observatory, held at the Pontifical Gregorian University in Rome, Italy, 12-16 June 2000, vol. 230 of ASP Conference Series, 247-256, (Astronomical Society of the Pacific, San Francisco, U.S.A., 2001). 6.1

[143] Kotake, K., Katsuhiko, S., and Keitaro, T., "Explosion Mechanism, Neutrino Burst, and Gravitational Wave in Core-Collapse Supernovae", Rep. Prog. Phys., submitted, (2005). Related online version (cited on 21 December 2005):

http://arXiv.org/abs/astro-ph/0509456. 3.1

[144] Kotake, K., Yamada, S., and Sato, K., "Gravitational radiation from axisymmetric rotational core collapse", Phys. Rev. D, 68, 044023, (2003). 3.4.2, 3.4.2

[145] Kotake, K., Yamada, S., Sato, K., Sumiyoshi, K., Ono, H., and Suzuki, H., "Gravitational radiation from rotational core collapse: Effects of magnetic fields and realistic equations of state", Phys. Rev. D, 69, 124004-1-11, (2004). 3.4 .2

[146] Lai, D., "Secular bar-mode evolution and gravitational waves from neutron stars", in Centrella, J.M., ed., Astrophysical Sources for Ground-based Gravitational Wave Detectors, Philadelphia, Pennsylvania, USA, 30 October - 1 November 2000, vol. 575 of AIP Conference Proceedings, 246-257, (American Institute of Physics, Melville, U.S.A., 2001). 3.4.4, 7

[147] Lai, D., and Goldreich, P., "Growth of perturbations in gravitational collapse and accretion", Astrophys. J., 535, 402-411, (2000). Related online version (cited on 25 June 1999): http://arXiv.org/abs/astro-ph/9906400. 3.4.5

[148] Lai, D., and Shapiro, S.L., "Gravitational radiation from rapidly rotating nascent neutron stars", Astrophys. J., 442, 259-272, (1995). Related online version (cited on 17 August 1994): http://arXiv.org/abs/astro-ph/9408053. 3.4.4

[149] Leaver, E.W., "An analytic representation for the quasi-normal modes of Kerr black holes", Proc. R. Soc. London, Ser. A, 402, 285-298, (1985). 6.4

[150] Liebendörfer, M., Messer, O.E.B., Mezzacappa, A., Bruenn, S.W., Cardall, C.Y., and Thielemann, F.-K., "A finite difference representation of neutrino radiation hydrodynamics for spherically symmetric general relativistic supernova simulations", Astrophys. J. Suppl. Ser., 150, 263-316, (2004). 7 
[151] Lindblom, L., and Owen, B.J., "Effect of hyperon bulk viscosity on neutron-star r-modes", Phys. Rev. D, 65, 063006-1-15, (2002). Related online version (cited on 25 October 2001): http://arXiv.org/abs/astro-ph/0110558. 2.3

[152] Lindblom, L., Owen, B.J., and Morinsk, S.M., "Gravitational radiation instability in hot young stars", Phys. Rev. Lett., 80, 4843-4846, (1998). Related online version (cited on 13 March 1998):

http://arXiv.org/abs/gr-qc/9803053. 2.3

[153] Lindblom, L., Tohline, J.E., and Vallisneri, M., "Nonlinear evolution of the $r$-modes in neutron stars", Phys. Rev. Lett., 86, 1152-1155, (2001). Related online version (cited on 31 October 2000):

http: //arXiv.org/abs/astro-ph/0010653. 2.3, 3.4.4

[154] Lindblom, L., Tohline, J.E., and Vallisneri, M., "Numerical evolutions of nonlinear $r$-modes in neutron stars", Phys. Rev. D, 65, 084039-1-15, (2002). Related online version (cited on 20 September 2001):

http://arXiv.org/abs/astro-ph/0109352. 2.3

[155] Liu, Y.T., "Dynamical instability of new-born neutron stars as sources of gravitational radiation", Phys. Rev. D, 65, 124003-1-14, (2002). Related online version (cited on 21 September 2001): http://arXiv.org/abs/gr-qc/0109078. 2.4.2, 7

[156] Liu, Y.T., and Lindblom, L., "Models of rapidly rotating neutron stars: remnants of accretion-induced collapse", Mon. Not. R. Astron. Soc., 324, 1063-1073, (2001). Related online version (cited on 9 December 2000):

http://arXiv.org/abs/astro-ph/0012198. 2.1, 2.4.2

[157] Loeb, A., and Rasio, F.A., "Collapse of primordial gas clouds and the formation of quasar black holes", Astrophys. J., 432, 52-61, (1994). Related online version (cited on 16 January 1994): http://arXiv.org/abs/astro-ph/9401026. 6.1

[158] Loveridge, L.C., "Gravitational waves from a pulsar kick caused by neutrino conversions", Phys. Rev. D, 69, 024008, (2004). 3.3

[159] Macchetto, F.D., "Supermassive black holes and galaxy morphology", Astrophys. Space Sci., 269, 269-291, (1999). Related online version (cited on 5 October 1999): http://arXiv.org/abs/astro-ph/9910089. 6.1

[160] MacFadyen, A.I., and Woosley, S.E., "Collapsars: Gamma-Ray Bursts and Explosions in "Failed Supernovae"”, Astrophys. J., 524, 262-289, (1999). 4.1

[161] Maiolino, R., Vanzi, L., Mannucci, F., Cresci, G., Ghinassi, F., and Della Valle, M., "Discovery of two infrared supernovae: a new window on the SN search", Astron. Astrophys., 389, 84-92, (2002). 3.2

[162] Managan, R.A., "On the secular instability of axisymmetric rotating stars to gravitational radiation reaction", Astrophys. J., 294, 463-473, (1985). 2.3

[163] Marck, J.-A., and Bonazzola, S., "Gravitational radiation from three-dimensional gravitational stellar core collapse", in D'Inverno, R., ed., Approaches to Numerical Relativity, Proceedings of the International Workshop on Numerical Relativity, Southampton, England, 16-20 December 1991, 247, (Cambridge University Press, Cambridge, U.K., 1992). 3.4.3

Living Reviews in Relativity

http://www. livingreviews.org/lrr-2003-2 
[164] Max Planck Institute for Astrophysics, "MPA Hydro Gang Homepage", project homepage. URL (cited on 17 April 2002):

http://www.mpa-garching.mpg.de/Hydro/index.shtml. url updated in 2006 revision. 3.4 .4

[165] Max Planck Institute for Astrophysics, "General relativistic simulations of rotational supernova collapse", project homepage, (2002). URL (cited on 3 July 2002):

http://www.mpa-garching.mpg.de/rel_hydro/axi_core_collapse/index.shtml. url updated in 2006 revision. 3.4 .4

[166] Mayle, R., and Wilson, J.R., "Supernovae from collapse of oxygen-magnesium-neon cores", Astrophys. J., 334, 909-926, (1988). 2.4 .1

[167] Mezzacappa, A., Calder, A.C., Bruenn, S.W., Blondin, J.M., Guidry, M.W., Strayer, M.R., and Umar, A.S., "The interplay between proto-neutron star convection and neutrino transport in core collapse supernovae", Astrophys. J., 493, 848-862, (1998). Related online version (cited on 18 September 1997): http://arXiv.org/abs/astro-ph/9709184. 3.1

[168] Mezzacappa, A., Calder, A.C., Bruenn, S.W., Blondin, J.M., Guidry, M.W., Strayer, M.R., and Umar, A.S., "An investigation of neutrino-driven convection and the core collapse supernova mechanism using multigroup neutrino transport", Astrophys. J., 495, 911-926, (1998). Related online version (cited on 18 September 1997):

http://arXiv.org/abs/astro-ph/9709188. 3.1

[169] Misner, C.W., Thorne, K.S., and Wheeler, J.A., Gravitation, (Freeman, New York, U.S.A., 1973). $\quad 2.4 .1$

[170] Mönchmeyer, R., Schäfer, G., Müller, E., and Kates, R.E., "Gravitational waves from the collapse of rotating stellar cores", Astron. Astrophys., 246, 417-440, (1991). 1, 2.4.1, 3.1, $3.3,3.4 .2,3.4 .2$

[171] Müller, E., "Gravitational radiation from collapsing rotating stellar cores", Astron. Astrophys., 114, 53-59, (1982). 1, 3.4.1, 3.4.2

[172] Müller, E., "Gravitational waves from core collapse supernovae", in Marck, J.-A., and Lasota, J.-P., eds., Relativistic Gravitation and Gravitational Radiation, Proceedings of the Les Houches School of Physics, held in Les Houches, Haute Savoie, 26 September - 6 October, 1995, Cambridge Contemporary Astrophysics, 273-308, (Cambridge University Press, Cambridge, U.K., 1997). 1

[173] Müller, E., "Simulation of astrophysical fluid flow", in LeVeque, R.J., Mihalas, D., Dorfi, E.A., and Müller, E., eds., Computational Methods for Astrophysical Fluid Flow, Lecture Notes 1997 of the Swiss Society for Astronomy and Astrophysics (SSAA), held March 38, 1997 in Les Diablerets, Switzerland, vol. 27 of Saas-Fee Advanced Courses, 343-494, (Springer, Berlin, Germany; New York, U.S.A., 1998). 1, 3.1, 3.4.5

[174] Müller, E., and Hillebrandt, W., "The collapse of rotating stellar cores", Astron. Astrophys., 103, 358-366, (1981). 3.4 .1

[175] Müller, E., Hillebrandt, W., and Rozyczka, M., "Stellar Collapse - Adiabatic hydrodynamics and shock wave propagation", Astron. Astrophys., 81, 288-292, (1980). 3.4 .2 
[176] Müller, E., and Janka, H.-T., "Gravitational radiation from convective instabilities in Type II supernova explosions", Astron. Astrophys., 317, 140-163, (1997). 3.3, 3.4.5, 3.4.5, 14, $15,3.4 .5$

[177] Müller, E., Rampp, M., Buras, R., Janka, H.-T., and Shoemaker, D.H., "Toward Gravitational Wave Signals from Realistic Core-Collapse Supernova Models", Astrophys. J., 603, 221-230, (2004). 3.4.2, 3.4.5, 3.4.5

[178] Nadyozhin, D.K., "Physical Properties of SNe IIP Derived from a Comparison of Theoretical Models with Observations", Astron. Astrophys., submitted, (2002). Related online version (cited on 09 January 2006):

http://www . mpa-garching.mpg.de/mpa/publications/preprints/pp2002/pp2002-en. html. 3.1

[179] Nakamura, T., "General relativistic collapse of axially symmetric stars leading to the formation of rotating black holes", Prog. Theor. Phys., 65, 1876-1890, (1981). 1, 4.4

[180] Nakamura, T., "General Relativistic Collapse of Accreting Neutron Stars with Rotation", Prog. Theor. Phys., 70, 1144-1147, (1983). 4.4

[181] NASA/ESA, "Laser Interferometer Space Antenna", project homepage. URL (cited on 4 October 2002):

http://lisa.jpl.nasa.gov. 1

[182] National Astronomical Observatory, "TAMA Project", project homepage. URL (cited on 4 October 2002):

http://tamago.mtk.nao.ac.jp/. 1

[183] Nazin, S.N., and Postnov, K.A., "High neutron star birth velocities and gravitational radiation during supernova explosions", Astron. Astrophys., 317, L79-L81, (1997). Related online version (cited on 15 January 1997):

http: //arXiv.org/abs/astro-ph/9701073. 3.4.5

[184] New, K.C.B., Centrella, J.M., and Tohline, J.E., "Gravitational waves from long-duration simulations of the dynamical bar instability", Phys. Rev. D, 62, 064019-1-16, (2000). Related online version (cited on 30 November 1999):

http://arXiv.org/abs/astro-ph/9911525. 2.4 .2

[185] New, K.C.B., and Shapiro, S.L., "Evolution of differentially rotating supermassive stars to the onset of bar instability", Astrophys. J., 548, 439-446, (2001). Related online version (cited on 9 October 2000):

http://arXiv.org/abs/astro-ph/0010172. 2.4.2, 6.1

[186] New, K.C.B., and Shapiro, S.L., "The formation of supermassive black holes and the evolution of supermassive stars", Class. Quantum Grav., 18, 3965-3976, (2001). Related online version (cited on 6 September 2000):

http://arXiv.org/abs/astro-ph/0009095. 6.1

[187] Nomoto, K., and Kondo, Y., "Conditions for accretion-induced collapse of white dwarfs", Astrophys. J. Lett., 367, L19-L22, (1991). 2.1, 1

[188] Novikov, I.D., "Gravitational radiation from a star collapsing into a disk", Sov. Astron., 19, 398, (1975). 3.4 .1

[189] Oshea, B.W., Norman M., personal communication, (2005). 5.2

Living Reviews in Relativity

http://www. livingreviews.org/lrr-2003-2 
[190] Ott, C.D., Burrows, A., Livne, E., and Walder, R., "Gravitational Waves from Axisymmetric, Rotating Stellar Core Collapse", Astrophys. J., 600, 834-864, (2004). 3.4 .2

[191] Ou, S., Tohline, J.E., and Lindblom, L., "Supernovae and the nuclear equation of state at high densities", Astrophys. J., 617, 490-499, (2004). 3.4.4, 6

[192] Pickett, B.K., Durisen, R.H., and Davis, G., "The dynamic stability of rotating protostars and protostellar disks. I. The effects of the angular momentum distribution", Astrophys. J., 458, 714-738, (1996). $\quad 2.3,2.4 .2$

[193] Piran, T., and Stark, R.F., "Numerical relativity, rotating gravitational collapse, and gravitational radiation", in Centrella, J.M., ed., Dynamical Spacetimes and Numerical Relativity, Proceedings of a workshop held at Drexel University, October 7-11, 1985, 40-73, (Cambridge University Press, Cambridge, U.K.; New York, U.S.A., 1986). 3.4.1

[194] Podsiadlowski, P., Joss, P.C., and Hsu, J.J.L., "Presupernova evolution in massive interacting binaries", Astrophys. J., 391, 246-264, (1992). 3.1

[195] Popham, R., Woosley, S.E., and Fryer, C.L., "Hyperaccreting Black Holes and Gamma-Ray Bursts", Astrophys. J., 518, 356-374, (1999). 4.1

[196] Proga, D., MacFadyen, A.I., Armitage, P.J., and Begelman, M.C., "Axisymmetric Magnetohydrodynamic Simulations of the Collapsar Model for Gamma-Ray Bursts", Astrophys. J. Lett., 599, L5-L8, (2003). 4.1

[197] Rampp, M., and Janka, H.-T., "Radiation hydrodynamics with neutrinos: Variable Eddington factor method for core-collapse supernova simulations", Astron. Astrophys., 396, 361-392, (2002). Related online version (cited on 7 March 2002): http://arXiv.org/abs/astro-ph/0203101. 3.1, 7

[198] Rampp, M., Müller, E., and Ruffert, M., "Simulations of non-axisymmetric rotational core collapse", Astron. Astrophys., 332, 969-983, (1998). Related online version (cited on 11 November 1997):

http://arXiv.org/abs/astro-ph/9781112. 1, 3.4.3, 3.4.4, 7

[199] Rees, M.J., "Astrophysical Evidence for Black Holes", in Wald, R.M., ed., Black Holes and Relativistic Stars, Proceedings of the Symposium dedicated to the memory of Subrahmanyan Chandrasekhar, held in Chicago, December 14-15, 1996, 79-101, (University of Chicago Press, Chicago, U.S.A.; London, U.K., 1998). 6.1

[200] Rezzolla, L., "Relativistic Astrophysics movies at SISSA", personal homepage, SISSA / ISAS. URL (cited on 4 October 2002):

http://www.sissa.it/〜rezzolla/movies.html. 4.4

[201] Rezzolla, L., Lamb, F.K., Marković, D., and Shapiro, S.L., "Properties of $r$ modes in rotating magnetic neutron stars. I. Kinematic secular effects and magnetic evolution", Phys. Rev. D, 64, 104013-1-12, (2001). Related online version (cited on 17 July 2001): http://arXiv.org/abs/gr-qc/0107061. 2.3

[202] Rezzolla, L., Lamb, F.L., Markovic, D., and Shapiro, S.L., "Properties of $r$ modes in rotating magnetic neutron stars. II. Evolution of the $r$ modes and stellar magnetic field", Phys. Rev. $D$, 64, 104014-1-13, (2001). Related online version (cited on 17 July 2001): http://arXiv.org/abs/gr-qc/0107062. 2.3 
[203] Ruffini, R., and Wheeler, J.A., "Relativistic Cosmology from Space Platforms", in Hardy, V., and H. Moore, H., eds., Proceedings of the Conference on Space Physics, 45-174, (ESRO, Paris, France, 1971). 1, 3.4.1

[204] Saenz, R.A., and Shapiro, S.L., "Gravitational radiation from stellar collapse - Ellipsoidal models", Astrophys. J., 221, 286-303, (1978). 1, 3.4.1

[205] Saenz, R.A., and Shapiro, S.L., "Gravitational and neutrion radiation from stellar core collapse - Improved ellipsoidal model calculations", Astrophys. J., 229, 1107-1125, (1979). 3.4.1

[206] Saenz, R.A., and Shapiro, S.L., "Gravitational radiation from stellar core collapse. III Damped ellipsoidal oscillations", Astrophys. J., 244, 1033-1038, (1981). 3.4 .1

[207] Saijo, M., "The Collapse of Differentially Rotating Supermassive Stars: Conformally Flat Simulations", Astrophys. J., 615, 866-879, (2004). 6.4

[208] Saijo, M., Baumgarte, T.W., Shapiro, S.L., and Shibata, M., "Collapse of a rotating supermassive star to a supermassive black hole: Post-Newtonian simulations", Astrophys. J., 569, 349-361, (2002). Related online version (cited on 6 February 2002):

http://arXiv.org/abs/astro-ph/0202112. 6.4, 19, 7

[209] Saijo, M., Shibata, M., Baumgarte, T.W., and Shapiro, S.L., "Dynamical bar instability in rotating stars: effect of general relativity", Astrophys. J., 548, 919-931, (2001). Related online version (cited on 10 October 2000):

http://arXiv.org/abs/astro-ph/0010201. 2.3

[210] Salpeter, E.E., "Energy and pressure of a zero-temperature plasma", Astrophys. J., 134, 669-682, (1961). 2.4.2

[211] Sanders, R.H., "The effects of stellar collisions in dense stellar systems", Astrophys. J., 162, 791-809, (1970). 6.1

[212] Scheck, L., Plewa, T., Janka, H.-T., Kifonidis, K., and Müller, E., "Pulsar Recoil by LargeScale Anisotropies in Supernova Explosions", Phys. Rev. Lett., 92, 011103, (2004). 3.1, 3.4.5

[213] Schenk, A.K., Arras, P., Flanagan, É.É., Teukolsky, S.A., and Wasserman, I., "Nonlinear mode coupling in rotating stars and the $r$-mode instability in neutron stars", Phys. Rev. D, 65, 024001-1-43, (2002). Related online version (cited on 23 January 2001):

http://arXiv.org/abs/gr-qc/0101092. 2.3

[214] Schutz, B.F., "Gravitational Wave Astronomy", Class. Quantum Grav., 16, A131-A156, (1999). Related online version (cited on 9 November 1999):

http://arXiv.org/abs/gr-qc/9911034.

[215] Seidel, E., and Moore, T., "Gravitational radiation from realistic relativistic stars: Oddparity fluid perturbations", Phys. Rev. D, 35, 2287-2296, (1987). 3.4.1

[216] Seidel, E., and Moore, T., "Gravitational radiation from perturbations of stellar core collapse models", in Evans, C.R., Finn, L.S., and Hobill, D.W., eds., Frontiers in Numerical Relativity, 146-162, (Cambridge University Press, Cambridge, U.K.; New York, U.S.A., 1988). 3.4.1

[217] Sekiguchi, Y., and Shibata, M., "New criterion for direct black hole formation in rapidly rotating stellar collapse", Phys. Rev. D, 70, 084005, (2004). 4.4

Living Reviews in Relativity

http: //www . livingreviews . org/lrr-2003-2 
[218] Shapiro, S.L., "Gravitational radiation from stellar collapse - The initial burst", Astrophys. J., 214, 566-575, (1977). 3.4 .1

[219] Shapiro, S.L., and Lightman, A.P., "Rapidly rotating, post-Newtonian neutron stars", Astrophys. J., 207, 263-278, (1976). 3.4.2

[220] Shapiro, S.L., and Teukolsky, S.A., "Gravitational collapse of supermassive stars to black holes - Numerical solution of the Einstein equations", Astrophys. J., 234, L177-L181, (1979). 6.4

[221] Shapiro, S.L., and Teukolsky, S.A., Black Holes, White Dwarfs, and Neutron Stars, (Wiley, New York, U.S.A., 1983). 2.3, 6.1

[222] Shibata, M., "Axisymmetric Simulations of Rotating Stellar Collapse in Full General Relativity - Criteria for Prompt Collapse to Black Holes -", Prog. Theor. Phys., 104, 325-358, (2000). Related online version (cited on 19 July 2000):

http://arXiv.org/abs/gr-qc/0007049. 4.4, 7

[223] Shibata, M., Baumgarte, T.W., and Shapiro, S.L., "The bar-mode instability in differentially rotating neutron stars: Simulations in full general relativity", Astrophys. J., 542, 453-463, (2000). Related online version (cited on 18 May 2000):

http://arXiv.org/abs/astro-ph/0005378. 2.3

[224] Shibata, M., Baumgarte, T.W., and Shapiro, S.L., "Stability and collapse of rapidly rotating, supramassive neutron stars: 3D simulations in general relativity", Phys. Rev. D, 61, 044012 1-11, (2000). Related online version (cited on 16 November 1999):

http://arXiv.org/abs/astro-ph/9911308. 2.5

[225] Shibata, M., and Karino, S., "Numerical evolution of secular bar-mode instability induced by gravitational radiation reaction in rapidly rotating neutron stars", Phys. Rev. D, 70, 084022-1-15, (2004). 2.3

[226] Shibata, M., Karino, S., and Eriguchi, Y., "Dynamical instability of differentially rotating stars", Mon. Not. R. Astron. Soc., 334, L27-L32, (2002). Related online version (cited on 6 June 2002):

http://arXiv.org/abs/gr-qc/0206002. 2.3

[227] Shibata, M., Karino, S., and Eriguchi, Y., "Dynamical bar-mode instability of differentially rotating stars: effects of equations of state and velocity profiles", Mon. Not. R. Astron. Soc., 343, 619-626, (2003). 2.3

[228] Shibata, M., and Shapiro, S.L., "Collapse of a rotating supermassive star to a supermassive black hole: Fully relativistic simulations", Astrophys. J. Lett., 572, L39-L43, (2002). URL (cited on 07 May 2002):

http://arXiv.org/abs/astro-ph/0205091. 6.4

[229] Shibata, M., Shapiro, S.L., and Uryu, K., "Equilibrium and stability of supermassive stars in binary systems", Phys. Rev. D, 64, 24004-1-14, (2001). Related online version (cited on 25 April 2001): http://arXiv.org/abs/astro-ph/0104408. 6.4

[230] Shibata, M., and Yu-ichirou, S., "Gravitational waves from axisymmetric rotating stellar core collapse to a neutron star in full general relativity", Phys. Rev. D, 69, 084024-1-16, (2004). 3.4.4 
[231] Smith, S., Houser, J.L., and Centrella, J.M., "Simulations of nonaxisymmetric instability in a rotating star: A comparison between Eulerian and Smooth Particle Hydrodynamics", Astrophys. J., 458, 236-256, (1996). Related online version (cited on 9 October 1995): http://arXiv.org/abs/gr-qc/9510014. 2.4.2

[232] Spruit, H.C., "Dynamo action by differential rotation in a stably stratified stellar interior", Astron. Astrophys., 381, 923-932, (2002). 3.4 .4

[233] Stark, R.F., and Piran, T., "Gravitational wave emission from rotating gravitational collapse", Phys. Rev. Lett., 55, 891-894, (1985). 1, 3.4.1, 6.4

[234] Starrfield, S., Timmes, F.X., Hix, W.R., Sion, E.M., Sparks, W.M., and Dwyer, S.J., "Surface Hydrogen-burning Modeling of Supersoft X-ray Binaries: Are they Type Ia Supernovae Progenitors?", Astrophys. J., 612, L53-L56, (2004). 2.2

[235] Stergioulas, N., Apostolatos, T.A., and Font, J.A., "Non-linear pulsations in differentially rotating neutron stars: mass-shedding-induced damping and splitting of the fundamental mode", Mon. Not. R. Astron. Soc., 352, 1089-1101, (2004). 2.3, 3.4.4

[236] Stergioulas, N., and Font, J.A., "Nonlinear $r$-modes in rapidly rotating relativistic stars", Phys. Rev. Lett., 86, 1148-1151, (2001). Related online version (cited on 31 July 2000): http://arXiv.org/abs/gr-qc/0007086. 2.3

[237] Swesty, F.D., Lattimer, J.M., and Myra, E.S., "The role of the equation of state in the 'prompt' phase of type II supernovae", Astrophys. J., 425, 195-204, (1994). 3.1

[238] Symbalisty, E.M.D., "Magnetorotational iron core collapse", Astrophys. J., 285, 729-746, (1984). $\quad 3.4 .2$

[239] Tassoul, J.-L., Theory of Rotating Stars, (Princeton University Press, Princeton, U.S.A., 1978). $\quad 2.3,3.4 .4$

[240] Thompson, T.A., Burrows, A., and Pinto, P.A., "Shock breakout in core-collapse supernovae and its neutrino signature", Astrophys. J., 592, 434-456, (2003). URL (cited on 10 November 2002):

http://arXiv.org/abs/astro-ph/0211194. 7

[241] Thorne, K.S., "Multipole expansion of gravitational radiation", Rev. Mod. Phys., 52, 299$340,(1980) . \quad 3.4 .2,3.4 .5$

[242] Thorne, K.S., "Gravitational Radiation", in Hawking, S.W., and Israel, W., eds., Three Hundred Years of Gravitation, 330-458, (Cambridge University Press, Cambridge, U.K.; New York, U.S.A., 1987). 6.4

[243] Thorne, K.S., "Gravitational radiation", in Böhringer, H., Morfill, G.E., and Trümper, J.E., eds., 17th Texas Symposium on Relativistic Astrophysics and Cosmology, vol. 759 of Annals of the New York Academy of Sciences, 127-152, (New York Academy of Sciences, New York, U.S.A., 1995). 6.4

[244] Thorne, K.S., "Gravitational Waves from Compact Bodies", in van Paradijs, J., van den Heuvel, E.P.J., and Kuulkers, E., eds., Compact Stars in Binaries, Proceedings of the 165th Symposium of the International Astronomical Union, held in The Hague, the Netherlands, August 15-19, 1994, vol. 165 of IAU Symposia, 153-184, (Kluwer Academic Publishers, Dordrecht, Netherlands; Boston, U.S.A., 1996). Related online version (cited on 30 June 1995):

http://arXiv.org/abs/gr-qc/9506084. 1 
[245] Thuan, T.X., and Ostriker, J.P., "Gravitational radiation from stellar collapse", Astrophys. J. Lett., 191, L105-L107, (1974). 1, 3.4.1

[246] Tohline, J.E., "The collapse of rotating stellar cores - Equilbria between white dwarf and neutron star densities", Astrophys. J., 285, 721-728, (1984). 3.4.2

[247] Tohline, J.E., and Hachisu, I., "The breakup of self-gravitating rings, tori, and thick accretion disks", Astrophys. J., 361, 394-407, (1990). 2.3, 2.4.2

[248] Toman, J., Imamura, J.N., Pickett, B.K., and Durisen, R.H., "Nonaxisymmetric dynamic instabilities of rotating polytropes. I. The Kelvin modes", Astrophys. J., 497, 370-387, (1998). 2.3

[249] Turner, M.S., and Wagoner, R.V., "Gravitational radiation from slowly-rotating supernovae - Preliminary results", in Smarr, L.L., ed., Sources of Gravitational Radiation, Proceedings of the Battelle Seattle Workshop, July 24 - August 4, 1978, 383-407, (Cambridge University Press, Cambridge, U.K., 1979). 3.4 .1

[250] van Putten, M.H.P.M., "Gravitational Wave Frequencies and Energies in Hypernovae", Astrophys. J., 583, 374-378, (2003). 4.3

[251] van Putten, M.H.P.M., and Levinson, A., "Theory and astrophysical consequences of a magnetized torus around a rapidly rotating black hole", Astrophys. J., 584, 937-953, (2003). URL (cited on 12 December 2002):

http://arXiv.org/abs/astro-ph/0212297. 4.3, 4.4, 7

[252] Villain, L., Pons, J.A., Cerdá-Durán, P., and Gourgoulhon, E., "Evolutionary sequences of rotating protoneutron stars", Astron. Astrophys., 418, 283-294, (2004). 2.3

[253] Walder, R., Burrows, A., Ott, C.D., Livne, E., Lichtenstadt, I., and Jarrah, M., "Anisotropies in the Neutrino Fluxes and Heating Profiles in Two-dimensional, Time-dependent, Multigroup Radiation Hydrodynamics Simulations of Rotating Core-Collapse Supernovae", Astrophys. J., 626, 317-332, (2005). 3.1

[254] Watts, A.L., Andersson, N., and Jones, D.I., "The Nature of Low $T /|W|$ Dynamical Instabilities in Differentially Rotating Stars", Astrophys. J. Lett., 618, L37-L40, (2005). 2.3

[255] Wheeler, J.C., Yi, I., Höflich, P., and Wang, L., "Asymmetric Supernovae, Pulsars, Magnetars, and Gamma-Ray Bursts", Astrophys. J., 537, 810-823, (2000). 3.1

[256] Wickramasinghe, D.T., and Ferrario, L., "Magnetism in Isolated and Binary White Dwarfs", Publ. Astron. Soc. Pac., 112, 873-924, (2000). 2.4 .2

[257] Wilson, J.R., "title missing", in Centrella, J.M., LeBlanc, J.M., and Bowers, J.L., eds., Numerical Astrophysics, Proceedings of a symposium in honor of James R. Wilson, held at the University of Illinois in October, 1982, 422-434, (Jones and Barlett, Boston, U.S.A., 1985). 3.1

[258] Wilson, J.R., and Mayle, R., "Convection in core collapse supernovae", Phys. Rep., 163, 63-78, (1988). 3.1

[259] Wilson, J.R., Mayle, R., Woosley, S.E., and Weaver, T.A., "Stellar Core Collapse and Supernovae", Ann. N.Y. Acad. Sci., 470, 267-293, (1986). 3.1

[260] Woodward, J., Tohline, J.E., and Hachisu, I., "The stability of thick, self-gravitating disks in protostellar systems", Astrophys. J., 420, 247-267, (1994). 2.3, 2.4.2 
[261] Woosley, S.E., "Gamma-Ray Bursts From Stellar Mass Accretion Disks Around Black Holes", Astrophys. J., 405, 273-277, (1993). 1

[262] Woosley, S.E., "Gamma-ray bursts from stellar mass accretion disks around black holes", Astrophys. J., 405, 273-277, (1993). 3.1, 4.1

[263] Woosley, S.E., and Baron, E., "The collapse of white dwarfs to neutron stars", Astrophys. J., 391, 228-235, (1992). 2.1, 2.4.1

[264] Yamada, S., and Sato, K., "Gravitational radiation from rotational collapse of a supernova core", Astrophys. J., 450, 245-252, (1995). 3.4 .2

[265] Yooun, S.C., and Langer, N., "Evolution of rapidly rotating metal-poor massive stars towards gamma-ray bursts", Astron. Astrophys., 443, 643-648, (2005). 7

[266] Young, P.A., Fryer, C.L., Hungerford, A., Arnett, D., Rockefeller, G., Timmes, F.X., Voit, B., Meakin, C., and Eriksen, K.A., "Constraints on the Progenitor of Cassiopeia A", Astrophys. $J$. , accepted, (2005). Related online version (cited on 21 December 2005):

http://arXiv.org/abs/astro-ph/0511806. 3.1

[267] Yu-ichirou, S., and Shibata, M., "Axisymmetric collapse simulations of rotating massive stellar cores in full general relativity: Numerical study for prompt black hole formation", Phys. Rev. D, 71, 084013-1-30, (2005). 4.4

[268] Yungelson, L.R., and Livio, M., "Type Ia Supernovae: An Examination of Potential Progenitors and the Redshift Distribution", Astrophys. J., 497, 168-177, (1998). Related online version (cited on 18 November 1997):

http://arXiv.org/abs/astro-ph/9711201. 2.2

[269] Zanotti, O., Rezzolla, L., and Font, J.A., "Quasi-periodic accretion and gravitational waves from oscillating "toroidal neutron stars" around a Schwarzschild black hole", Mon. Not. $R$. Astron. Soc., 341, 832-848, (2003). 4.3, 4.4

[270] Zel'dovich, Y.B., and Novikov, I.D., Relativistic Astrophysics, vol. 1, (University of Chicago Press, Chicago, U.S.A., 1971). 6.1

[271] Zwerger, T., and Müller, E., "Dynamics and gravitational wave signature of axisymmetric rotational core collapse", Astron. Astrophys., 320, 209-227, (1997). 1, 2.4.1, 2.4.2, 3.4.2, 3, 3.4.2, $4,5,3.4 .2,3.4 .3,3.4 .4,3.4 .4,3.4 .5$

Living Reviews in Relativity

http://www. livingreviews.org/lrr-2003-2 